\title{
Growth and thermoelectric properties of FeSb2 films produced by pulsed laser deposition
}

\author{
Canulescu, Stela; SUN, Ye; Schou, Jørgen; Iversen, Bo Brummerstedt
}

Publication date:

2010

Document Version

Publisher's PDF, also known as Version of record

Link back to DTU Orbit

Citation (APA):

Canulescu, S., SUN, Y., Schou, J., \& Iversen, B. B. (2010). Growth and thermoelectric properties of FeSb2 films produced by pulsed laser deposition. Poster session presented at 7th International Conference on Photo-Excited Processes and Applications, Copenhagen, Denmark.

http://www.icpepa7.com/upload/institutter/com/micro/bookabstracts_280910_2.pdf

\section{General rights}

Copyright and moral rights for the publications made accessible in the public portal are retained by the authors and/or other copyright owners and it is a condition of accessing publications that users recognise and abide by the legal requirements associated with these rights.

- Users may download and print one copy of any publication from the public portal for the purpose of private study or research.

- You may not further distribute the material or use it for any profit-making activity or commercial gain

- You may freely distribute the URL identifying the publication in the public portal

If you believe that this document breaches copyright please contact us providing details, and we will remove access to the work immediately and investigate your claim. 


\section{ICPEPA-7}

7th International Conference on Photo-Excited Processes and Applications

15-20 August 2010

Copenhagen, Denmark

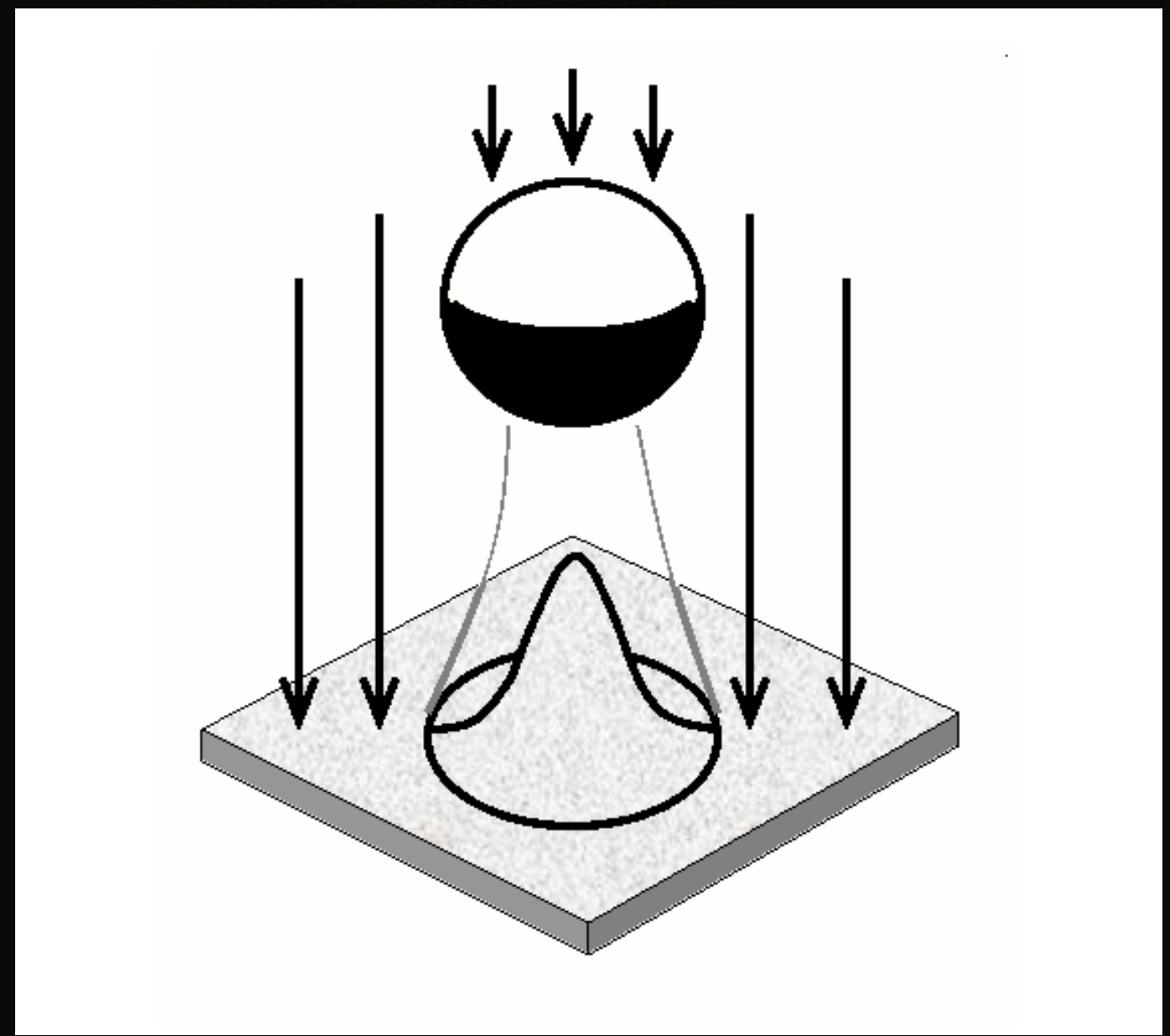




\section{ICPEPA 7 has received economic support from Carlsbergfondet, Toyota-fonden, Oticon Fonden and Otto Mønsted Fonden.}

The meeting has been arranged by the staff at DTU Fotonik, Niels Bohr Institute, Institute for Physics and Astronomy, University of Aarhus, and Mads Clausen Institute, SDU.

ICPEPA 7 gratefully acknowledges support from the exhibitors:

Azpect Photonics AB, FASTLITE, Thorlabs Sweden AB, FEMTOLASERS Produktions $\mathrm{GmbH}$, Gammadata Instrument $\mathrm{AB}$, Horiba Jobin Yvon GMBH and SURFACE systems + technology $\mathrm{GmbH}+\mathrm{Co} \mathrm{KG}$

\section{Local Committee}

Jørgen Schou (Technical University of Denmark)

Peter Balling (Århus University)

Martin M. Nielsen (University of Copenhagen)

Horst-Günter Rubahn(University of Southern Denmark)

\section{Conference secretaries}

Britt Boding (DTU Fotonik)

Bente Juhl Andersen (Niels Bohr Institute)

Zora Milde (Mads Clausen Institute) 


\title{
Steering Panel
}

\author{
Chunlei Guo, Rochester University, Rochester, USA \\ Peter Hess, University of Heidelberg, Germany \\ Aaron Peled, Holon Academic Inst. of Technol., Israel \\ Jørgen Schou, Technical University of Denmark, Denmark \\ Koji Sugioka, RIKEN, Japan \\ Leonid Zhigilei, University of Virginia, USA
}

\section{International Advisory Committee}

Susan Allen, Arkansas State Univ, USA

Salvatore Amoruso, CNR-INFM, University of Napoli, Italy

Micha Asscher, Hebrew Univ., Israel

Peter Balling, University of Århus, Denmark

Vitor Baranauskas, State Univ. of Campinas, Brasil

Anna Paola Caricato, University of Salento, Italy

Douglas Chrisey, Rensselaer Polytechnic Inst., USA

Thomas Dickinson, Washington State Univ., USA

Jan Dubowski, Univ. of Sherbrooke, Canada

Richard Haglund Jr., Vanderbilt Univ., USA

Jörg Hermann, CNRS/Univ. Aix-Marseille, France

Jürgen Ihlemann, Laser-Lab, Göttingen, Germany

Saulius Juodkazis, Swinburne Univ. of Techn., Australia

Safa Kasap, Saskatchewan Univ., Canada

Jôrgen Larsson, LTH, Lund Univ., Sweden

Thomas Lippert, Paul Scherrer Inst., Switzerland

Hiroshi Masuhara, Nara Institute of Sci. and Techn., Japan

Ion N. Mihailescu, NILPRP, Romania

Paolo Maria Ossi, The Polythechnic University of Milan, Italy

Gediminas Raciukaitis, Cent. Phys. Sci. Techn., Vilnius, Lithuania

Frank Träger, Univ. Kassel, Germany

Tetsuo Tsuchiya, Nat. Inst. of Adv. Ind. Sci. and Techn. , Japan

Xianfan Xu, Purdue Univ., USA 


\section{Complete Programme for ICPEPA 7}

(Invited talks $30 \mathrm{~min}$. incl. discussion, contributed talks $20 \mathrm{~min}$. incl. discussion.)

\section{Monday, 16 August, Copenhagen}

All oral presentations are given in

Lundbeck Auditorium, Biocenter, Ole Maaløes vej 5 , 2200 Copenhagen N

\begin{tabular}{|c|c|c|}
\hline \multirow[t]{2}{*}{$0800-0900$} & & Registration \\
\hline & & Chair : Jørgen Schou \\
\hline 0900-1015 & & $\begin{array}{l}900 \text { Opening of conference - } \\
\text { The 50-years anniversary of the laser } \\
905 \text { Opening by Rector, University of Copenhagen, Ralf Hemmingsen, } \\
920 \text { "Optical communication milestones and the laser", } \\
\text { Palle Jeppesen, and Christophe Peucheret, DTU Fotonik, } \\
\text { Technical University of Denmark, Denmark. } \\
940 \text { "The laser - the first } 50 \text { years", } \\
\text { Sune Svanberg, University of Lund, Lund, Sweden }\end{array}$ \\
\hline $1015-1045$ & & Coffee break \\
\hline & & Chair: Leonid Zhigilei \\
\hline $1045-1055$ & & $\begin{array}{l}\text { Practical comments on the conference } \\
\text { Jørgen Schou, DTU Fotonik, Denmark }\end{array}$ \\
\hline $1055-1250$ & $\begin{array}{l}\text { Key-note talk } \\
\text { Contr. 2-4 }\end{array}$ & $\begin{array}{l}\text { "Femtosecond electron diffraction phenomena" } \\
\text { Dwaine Miller, University of Toronto, Canada } \\
\text { "Fine-ripple formation at high fluence conditions during femtosecond }\end{array}$ \\
\hline
\end{tabular}




\begin{tabular}{|c|c|c|}
\hline & & $\begin{array}{l}\text { laser ablation of ceramics", } \\
\text { Sungho Leung, Gwangju Institute of Science and Technology (GIST)), } \\
\text { Gwangju, Republic of Korea } \\
\text { "Direct observation of acoustic oscillations in InAs nanowires", } \\
\text { Simon Mariager, University of Copenhagen, Copenhagen, Denmark } \\
\text { "Computer simulations of plume evolution in aluminium" } \\
\text { Johannes Roth, University of Stuttgart, Stuttgart, Germany }\end{array}$ \\
\hline \multirow[t]{2}{*}{$1250-1400$} & & Lunch \\
\hline & & Chair : Nini Pryds \\
\hline $1400-1540$ & $\begin{array}{l}\text { Invited } 1 \\
\text { Contr. 5-6 }\end{array}$ & $\begin{array}{l}\text { "Pulsed Laser deposition. Nucleation and growth on the atomic scale" } \\
\text { Peter Varga, Vienna University of Technology, Vienna, Austria } \\
\text { "Oxygen diffusion in SrTiO3 and LaAlO3 thin films prepared by pulsed } \\
\text { laser deposition “ } \\
\text { Thomas Lippert, PSI, Villigen, Switzerland } \\
\text { "Optical emission studies in pulsed Nd:YAG laser deposition of diamond- } \\
\text { like carbon", } \\
\text { Teck Yong Tou, Multimedia University, Cyberjaya, Malaysia, } \\
\text { "Functional interfaces in oxide heterostructures by atomically controlled } \\
\text { pulsed laser deposition”, } \\
\text { Guus Rijnders, University of Twente, Enschede, The Netherlands, }\end{array}$ \\
\hline \multirow[t]{2}{*}{$1540-1610$} & & Coffee break \\
\hline & & Chair: Motoichi Ohtsu \\
\hline $1610-1740$ & $\begin{array}{l}\text { Invited } 3 \\
\text { Contr. 7-9 }\end{array}$ & $\begin{array}{l}\text { "Ultrafast laser interaction with group IV materials" } \\
\text { Harold Haugen, McMaster University, Hamilton, Canada } \\
\text { "Nanocrystal growth of II-VI II-VI wide band gap semiconductors by ultra } \\
\text { fast laser ablation and deposition ", }\end{array}$ \\
\hline
\end{tabular}




\begin{tabular}{|c|c|}
\hline & $\begin{array}{l}\text { Marta Castillejo, Spanish Research Council, Madrid, Spain } \\
\text { "Experimental comparison between intensity and phase detection in } \\
\text { grating coupling surface plasmon resonance" } \\
\text { Wen-kai Kuo, National Formosa University, Taiwan } \\
\text { "Patterned laser annealing of silicon oxide films" } \\
\text { Jürgen Ihlemann, Laser Lab Göttingen, Göttingen, Germany }\end{array}$ \\
\hline $1740-1800$ & Break - walk to H.C. Ørsted Institute \\
\hline $1800-2030$ & Posters and exhibitions with beer and sandwiches \\
\hline 2000 & $\begin{array}{l}\text { Concert: } \\
\text { The Choir of Rigshospitalet sings traditional Danish Summer songs }\end{array}$ \\
\hline
\end{tabular}

\section{Tuesday, 17 August, Copenhagen}

All oral presentations are given in

Lundbeck Auditorium, Biocenter, Ole Maaløes vej 5 , 2200 Copenhagen N

\begin{tabular}{|c|c|c|}
\hline \multirow[t]{2}{*}{ 0830-0900 } & & Registration \\
\hline & & Chair: Martin Meedom Nielsen \\
\hline 0900-1040 & Invited 4 & $\begin{array}{l}\text { "Ultrafast processes and single shot imaging of clusters with intense X-ray } \\
\text { laser pulses" } \\
\text { Christoph Bostedt, SLAC, Stanford University, USA, } \\
\text { "Study of surface chemical reactions induced by molecules electronically } \\
\text { excited in the gas phase" } \\
\text { Victor Petrunin, University of Southern Denmark, Odense, Denmark } \\
\text { "Phase transition in carbon based material induced by intense }\end{array}$ \\
\hline
\end{tabular}




\begin{tabular}{|c|c|c|}
\hline & Invited 5 & $\begin{array}{l}\text { femtosecond x-ray pulse" } \\
\text { Jérôme Gaudin, European XFEL GmbH, Hamburg, Germany } \\
\text { "Ultrafast Delocalization of Hydrogen Atoms in Hydrocarbon Molecules in } \\
\text { Intense Laser Fields" } \\
\text { Kaoru Yamanouchi, University of Tokyo, Tokyo, Japan }\end{array}$ \\
\hline \multirow[t]{2}{*}{$1040-1110$} & & Coffee break \\
\hline & & Chair: James G. Lunney \\
\hline $1110-1240$ & Invited 6 & $\begin{array}{l}\text { "Atomistic simulations of laser interactions with metals: Melting of } \\
\text { nanocrystalline films and generation of crystal defects" } \\
\text { Leonid Zhigilei, University of Virginia, Charlottesville, USA } \\
\text { "The role of anisotropic excitation in self-organized nanostructure } \\
\text { formation upon femtosecond laser ablation" } \\
\text { Jürgen Reif, Brandenburgische Technische Universität, Cottbus, Germany } \\
\text { "The effect of laser wavelength on heating of the ablated plume", } \\
\text { Zygmunt Szymanski, Institute of Fundamental Technological Research, } \\
\text { Poland }\end{array}$ \\
\hline \multirow[t]{2}{*}{$1250-1400$} & & Lunch \\
\hline & & Chair: Peter Balling \\
\hline $1400-1530$ & $\begin{array}{l}\text { Invited } 7 \\
\text { Contr. 15,17 }\end{array}$ & $\begin{array}{l}\text { "Phonon-assisted nanophotonic fabrication and its application” } \\
\text { Motoichi Ohtsu, University of Tokyo, Tokyo, Japan } \\
\text { "Precipitation of ferromagnetic nanoparticles inside transparent glass by } \\
\text { femtosecond laser", } \\
\text { Seisuke Nakashima, RIKEN, Japan } \\
\text { "Dynamics of Exciton-Plasmon Coupling in ZnO-MgO-Metal } \\
\text { Heterostructures" } \\
\text { Richard Haglund, Vanderbilt University, Nashville, Tennessee, USA }\end{array}$ \\
\hline
\end{tabular}




\begin{tabular}{|c|c|c|}
\hline $1530-1600$ & & Coffee break \\
\hline & & Chair: Peter Hess \\
\hline $1600-1730$ & $\begin{array}{l}\text { Invited } 8 \\
\text { Contr. 18-20 }\end{array}$ & $\begin{array}{l}\text { "Nanoparticles produced by laser ablation" } \\
\text { Paolo M. Ossi, Politecnico di Milano, Milano, Italy } \\
\text { "Novel processing for the oxide films by photoreaction of nano-particles" } \\
\text { Tetsuo Tsuchiya, National Institute of Advanced Industrial Science and } \\
\text { Technology(AIST), Tsukuba, Japan } \\
\text { "Self-organised nanostructures on metal surfaces produced by } \\
\text { femtosecond laser ablation" } \\
\text { Evgeny Gurevich, ISAS, Institut fur Analytische Wissenschaften, } \\
\text { Dortmund, Germany". } \\
\text { "Selective Ablation of Xe on Silicon Surfaces: MD Simulation and } \\
\text { Experimental Laser Patterning", } \\
\text { Ori Stein, Hebrew University, Jerusalem, Israel }\end{array}$ \\
\hline $1730-1800$ & & Break - walk to H.C. $\emptyset$ rsted Institute \\
\hline $1800-2030$ & & Posters and exhibitions with beer and sandwiches \\
\hline
\end{tabular}

\section{Wednesday, 18 August, Copenhagen}

All oral presentations are given in

Lundbeck Auditorium, Biocenter, Ole Maaløes vej 5 , 2200 Copenhagen N

\begin{tabular}{|l|l|l|}
\hline & & Chair: Kaoru Yamanouchi \\
\hline $0900-1040$ & Invited 9 & "Laser interactions with organic MALDI matrices" \\
\hline
\end{tabular}




\begin{tabular}{|c|c|c|}
\hline & Contr. 21-22 & $\begin{array}{l}\text { Klaus Dreisewerd, University of Münster, Münster, Germany } \\
\text { "Investigating the fragmentation of C60 by Matrix Assisted Pulsed Laser } \\
\text { Evaporation (MAPLE)”, } \\
\text { Stela Canulescu, Technical University of Denmark, Roskilde, Denmark } \\
\text { “Mechanistic evaluation of } 213 \text { nm pulsed laser cornea ablation”, } \\
\text { Wolfgang Kautek, University of Vienna, Vienna, Austria } \\
\text { “Production of films of organic materials for biomedical applications by } \\
\text { laser-assisted methods” } \\
\text { Ion N. Mihailescu, National Institute for Lasers, Plasma and Radiation } \\
\text { Physics, Bucharest-Magurele, Romania }\end{array}$ \\
\hline $1040-1110$ & & Coffee break \\
\hline & & Chair: Marta Castillejo \\
\hline $1110-1300$ & $\begin{array}{l}\text { Invited } 11 \\
\text { Contr. 23-26 }\end{array}$ & $\begin{array}{l}\text { "Double pulse ultrafast laser ablation of metals" } \\
\text { Salvatore Amoruso, Università degli Studi di Napoli Federico II, } \\
\text { Napoli, Italy } \\
\text { "Pulsed laser deposition of La0.6Ca0.4O3: ion dynamics and thin } \\
\text { film properties", } \\
\text { Thomas Lippert, PSI, Villigen, Switzerland } \\
\text { "Electromagnetic control of plume expansion in laser ablation" } \\
\text { James G. Lunney, Trinity College Dublin, Dublin, Ireland } \\
\text { "Laser-induced breakdown spectroscopy for the analysis of } \\
\text { strontium and uranium in glass matrix" } \\
\text { E. C. Jung, Korea Atomic Energy Research Institute, Daejeon, } \\
\text { Republik of Korea } \\
\text { "Surface and bulk structuration of solid and liquid dielectrics", } \\
\text { Candice Mézel, CELIA, Talence, France }\end{array}$ \\
\hline
\end{tabular}




\begin{tabular}{|l|l|l|}
\hline $1300-1400$ & Lunch \\
\hline $1400-1700$ & Excursion to Copenhagen City - Harbour Cruise \\
\hline $1830-2300$ & $\begin{array}{l}\text { Reception and conference dinner in Stamhuset, Den danske } \\
\text { Frimurerorden, Blegdamsvej 23, 2100-Copenhagen } \varnothing\end{array}$ \\
\hline
\end{tabular}

\section{Thursday, 19 August, Copenhagen - Sønderborg}

All oral presentations are given in

Lundbeck Auditorium, Biocenter, Ole Maaløes vej 5, 2200 Copenhagen N

\begin{tabular}{|c|c|c|}
\hline & & Chair: Wolfgang Kautek \\
\hline $0900-1040$ & $\begin{array}{l}\text { Invited } 12 \\
\text { Contr. 27-28 } \\
\text { Invited } 13\end{array}$ & $\begin{array}{l}\text { "Structural dynamics in laser-excited materials studied with ultrafast X-ray } \\
\text { scattering” } \\
\text { Klaus Sokolowski-Tinten, Universität Duisburg-Essen, Duisburg, Germany } \\
\text { "Laser processing with short and ultrashort laser pulses” } \\
\text { Peter Balling, Århus University, Århus, Denmark } \\
\text { "Chemistry of monolayer functionalizations of silicon studied by laser- } \\
\text { induced desorption mass spectrometry” } \\
\text { Peter Hess, University of Heidelberg; Heidelberg, Germany. } \\
\text { „New opportunities at XFEL“ } \\
\text { Christian Bressler, XFEL, Hamburg, Germany }\end{array}$ \\
\hline $1040-1110$ & & Coffee break \\
\hline & & Chair: Jürgen Reif \\
\hline
\end{tabular}




\begin{tabular}{|c|c|c|}
\hline $1110-1240$ & Contr. 29-31 & $\begin{array}{l}\text { "Black and colored metals" } \\
\text { Chunlei Guo, University of Rochester, Rochester, USA } \\
\text { "Dielectric to metal transitions in silicon and dielectrics observed during } \\
\text { periodic surface structure formation induced by temporally tailored } \\
\text { pulses" } \\
\text { Magdalena Forster, University of Vienna, Vienna, Austria } \\
\text { "Synthesis of high-pressure phases of oxides by laser-induced optical } \\
\text { breakdown" } \\
\text { Vygantas Mizeikas, Shizuoka University, Japan } \\
\text { "Energy coupling localization and selective laser processing in thin-film } \\
\text { structures" } \\
\text { Gediminas Raciukaitis, Center for Physical Science and Technology, } \\
\text { Vilnius, Lithuania }\end{array}$ \\
\hline $1250-1400$ & & Lunch \\
\hline $1400-1800$ & & Bus departure and travelling to $\$ \varnothing$ nderborg \\
\hline $1800-1900$ & & Arrival at Hotels \\
\hline $1900-2200$ & & Social arrangement \\
\hline
\end{tabular}

\section{Friday, 20 August, Sønderborg}

All oral presentations are given in Alsion , Alsion 2, 6400 Sønderborg

\begin{tabular}{|l|l|l|}
\hline & & Chair: Jürgen Ihlemann \\
\hline 0830-1010 & Invited 15 & "Optical Near-Fields of Nanostructures: Characterization and Patterning" \\
& & Paul Leiderer, University of Konstanz, Konstanz, Germany \\
& Contr 32-33 & "Films of brookite titania nanorods deposited by matrix-assisted pulsed \\
\hline
\end{tabular}




\begin{tabular}{|c|c|c|}
\hline & Invited 16 & $\begin{array}{l}\text { laser deposition for gas sensor applications" } \\
\text { Anna Paola Caricato, University of Salento, Salento, Italy } \\
\text { "Characterization of laser induced crystallization processes of (Ag)-Sb-S } \\
\text { thin films towards optical data recording" } \\
\text { Jan Gutwirth, Faculty of Chemical Technology } \\
\text { University of Pardubice, Pardubice, Czech republic } \\
\text { "Adaptive nanooptics und spatiotemporal control" } \\
\text { Walter Pfeiffer, University of Bielefeld, Bielefeld, Germany }\end{array}$ \\
\hline $1010-1040$ & & Coffee break \\
\hline & & Chair: Gediminas Raciukaitis \\
\hline $1040-1210$ & $\begin{array}{l}\text { Invited } 17 \\
\text { Contr 34,36 }\end{array}$ & $\begin{array}{l}\text { "Ellipsometry of monolayers", } \\
\text { Peter Hess, University of Heidelberg, Heidelberg, Germany } \\
\text { Tailoring the optical properties of ZnO thin films by doping and co-doping } \\
\text { George Epurescu, NILPRP, Bucharest, Romania } \\
\text { NN } \\
\text { "Synthesis of gallium nitride nanocrystallites by pulsed laser ablation in } \\
\text { background gas of pure nitrogen", } \\
\text { Takehito Yoshida, Anan National College of Technology, Anan, } \\
\text { Tokushima, Japan }\end{array}$ \\
\hline $1210-1330$ & & Lunch \\
\hline $1330-1730$ & & $\begin{array}{l}\text { Bus departure and travelling to Copenhagen Airport/Copenhagen via } \\
\text { Fredericia Railway Station }\end{array}$ \\
\hline
\end{tabular}




\title{
Abstracts of Oral Presentations
}

\author{
(Monday, 16 August)
}

\author{
S1: The laser - the first 50 years \\ Presenter : Sune SVANBERG \\ Prof.. SVANBERG, Sune (Lund University, Sweden)
}

\section{S2: Femtosecond electron diffraction phenomena}

Presenter : Dwaine MILLER

Prof.. MILLER, Dwaine (University of Toronto, Canada)

\section{1: 3D nano- and mivrostructures in transparent materials by in-volume selective laser etching.}

Presenter : Jens GOTTMANN

Dr. GOTTMANN, Jens (RWTH Aachen University) HERMANS, Martin (RWTH Aachen University), HöRSTMANNJUNGEMANN, Maren (RWTH Aachen University), BECKMANN, Dennsi (RWTH Aachen University)

Self-organised In-volume Selective Laser-induced Etching (ISLE) is a manufacturing process for micro machining of transparent materials such as sapphire and glasses, e.g. fused silica and boro silicate glasses. By focusing the laser radiation in the volume the material is locally modified. By high speed scanning the laser focus with pulse overlap inside the material, connected volumes of modified material are created. The modified volumes are subsequently removed by chemical etching using aqueous solution of e.g. HF or KOH. Mirco channels, shaped holes and micro parts like gears are fabricated. Periodical nano structures (ripples, nanoplanes) are observed in sapphire in glasses with properties, which still cannot be predicted. The nanostructures are investigated using scanning electron microscopy and transmission electron microscopy. Amorphized nanoplanes and compositional changes are observed e.g. in sapphire. Pump-probe reflections spectroscopy, transmission spectroscopy and interference microscopy are used to investigate the transient refractive index of the materials during laser processing. Up to now, the results are in contradiction to several 
models describing the formation of nanoplanes or coherent sub-wavelength ripples. Furthermore, the pump probe investigations are useful to investigate the processing limits of ISLE, which are extended to scanning velocities $>1 \mathrm{~m} / \mathrm{s}$ exploiting high power $(\sim 100 \mathrm{~W})$ high repetition rate $(1-100 \mathrm{MHz}) \mathrm{fs}$ laser radiation.

\section{2: Fine-ripple formation at high fluence conditions during femtosecond laser ablation of ceramics}

Presenter : Sungho JEONG

Prof. JEONG, Sungho (Gwangju Institute of Science and Technology (GIST)) ; Mr. KIM, Sung Hoon (Gwangju Institute of Science and Technology (GIST)), Dr. SOHN, Ik-bu (Advanced Photonics Research Institute) Presenter : Prof. JEONG, Sungho (Gwangju Institute of Science and Technology (GIST))

Ripple formation during femtosecond laser ablation of solids have received significant attention in recent years due to its potential for direct fabrication of periodic nanostructures. The commonly observed laser-induced ripples are aligned in the direction perpendicular to the direction of laser polarization with a spacing comparable to the wavelength of incident laser light. Also, these ripples were typically known to be produced at laser fluencies near ablation threshold, often with multi-pulse ablation. However, newer types of ripple such as fine ripples whose spacing is much smaller than the laser wavelength, or parallel ripples whose direction is independent of laser polarization have also been reported in recent studies. The characteristics of these new ripples and the mechanisms of their formation are of great interest. In this work, we report the formation of fine ripples with a spacing of about $200 \mathrm{~nm}$ during femtosecond laser $(\lambda=785 \mathrm{~nm}, \tau=184 \mathrm{fs})$ ablation of ceramics. Especially, we observed the formation of fine ripples near the center of a crater at laser fluencies much higher than the ablation threshold. At this high irradiance condition, the chemistry of fine ripples near the crater center showed substantial difference from those of unablated region or crater edge region. Experimental observations indicated that the mechanisms of ripple formation at high fluence conditions differ from those of low fluence ripples, and should be explained in connection with 1 .

\section{3: Direct observation of acoustic oscillations in InAs nanowires}

\section{Presenter : Simon MARIAGER}

Mr. MARIAGER, Simon (Centre for Molecular Movies; Niels Bohr Institute), Prof. NIELSEN, Martin M. (Centre for Molecular Movies; Niels Bohr Institute) ; Prof. FEIDENHANS'L, Robert (Niels Bohr Institute) ; Mr. KHAKHULIN, Dmitry (Centre for Molecular Movies; Niels Bohr Insitute)

We have used time-resolved x-ray diffraction and optical reflectivity to directly measure three different acoustic oscillations of InAs nanowires. The oscillations are excited by a fs laser pulse and evolve at three different timescales. We measure the absolute scale of the initial radial expansion of the fundamental breathing eigenmode and determine the frequency by transient optical reflectivity. For the extensional eigenmode we measure the oscillations of the average radial and axial lattice constants and determine the amplitude of oscillations and the average extension. Finally we observe a bending motion of the nanowires. The frequencies of the eigen modes are in good agreements with predictions made by continuum elasticity theory and we find no difference in the speed of sound between the wurtzite nanowires and cubic bulk crystals, but the measured strain is 
influenced by the interaction between different modes, and differ slightly from the theoretical description.

\section{4: Computer Simulations of Plume Evolution in Aluminum}

Presenter : Johannes ROTH

Dr. ROTH, Johannes (University Stuttgart) Co-authors : Mr. SONNTAG, Steffen (University Stuttgart) ; Mrs. TRICHET PAREDES, Carolina (University Stuttgart) ; Prof. TREBIN, Hans-rainer (University Stuttgart)

We study femtosecond laser ablation and plume evolution of aluminum for various inhomogeneous laser pulses. For the simulations of the lattice we utilize molecular dynamics (MD) simulations using our own code. The gas-phase is then scanned by a cluster algorithm (DBSCAN), from which we gain a cluster distribution of the plume. Only a small portion of the whole sample evaporates into the gas phase, therefore we have to deal with huge (6 x $10^{\wedge} 7$ atoms) samples. The ablation threshold is determined by lemph \{measuring\} the diameter of the drilled hole and comparing it to the total pulse energy - similar to a method used also in experiment. Atomic scale images for both the plume evolution and the crater formation are shown.

\section{1: Pulsed Laser Deposition: Nucleation and Growth on the Atomic Scale (Invited)}

Presenter : Peter VARGA

Prof. VARGA, Peter (Institute of Applied Physics, Vienna University of Technology)

Co-authors : Prof. SCHMID, Michael (Institute of Applied Physics, Vienna University of Technology) ; Ms.LENAUER, Claudia (Institute of Applied Physics, Vienna University of Technology) ; Mr. WIMMER, Florian (Institute of Applied Physics, Vienna University of Technology) ; Prof. BETZ, Gerhard (Institute of Applied Physics, Vienna University of Technology) ; Prof. REDINGER, Josef (Institute of Applied Physics, Vienna University of Technology) ; Mr. BUCHSBAUM, Andreas (Institute of Applied Physics, Vienna University of Technology) ; Mr. GARHOFER, Andreas (Institute of Applied Physics, Vienna University of Technology) ; Mr. SCHEIBER, Philipp (Institute of Applied Physics, Vienna University of Technology)

Pulsed laser deposition (PLD) is a versatile deposition method that combines many features of technologically relevant processes such as sputter deposition with ultrahigh-vacuum compatibility and easy accessibility by surface science methods. PLD is characterized by high energies of the impinging particles (ions) and known to lead to higher island densities and better layer-by-layer growth than conventional thermal deposition. We have studied homoepitaxy of $\mathrm{Pt}$ on $\mathrm{Pt}(111)$ as well as heteroepitaxy of Co on $\mathrm{Pt}(111)$ by PLD with scanning tunneling microscopy (STM). For heteroepitaxy of $\mathrm{Co}$ on $\mathrm{Pt}(111)$, atomically resolved STM with chemical contrast shows implantation of impinging species in the surface. By directly watching diffusion processes, we demonstrate that the resulting heterogeneous surface impedes surface diffusion, leading to exceptionally high island densities. For homoepitaxy with moderate ion energies (\&lt; $100 \mathrm{eV})$, comparison with nucleation theory shows that the high island densities are merely caused by the high instantaneous flux of arriving particles, while the kinetic energy of the impinging particles plays no significant role. At higher ion energies (\&gt; $200 \mathrm{eV})$, however, additional nuclei are formed by "failed sputtering", i.e., formation of ad-atoms by the impinging energetic ions. Layer-by layer growth observed in PLD experiments down 
to very low temperatures can be explained by the small island size and implantation of energetic particles.

\section{5: Oxygen diffusion in SrTiO3 and LaAlO3 thin films prepared by pulsed laser deposition}

Presenter : Thomas Lippert

Dr. LIPPERT, Lippert Thomas (Paul Scherrer Institut), Mr. ESPOSITO, Martin (Paul Scherrer Institut) ; Dr. MAROZAU, Ivan (Paul Scherrer Institut) ; Dr. DOEBELI, Max (ETH Zurich) ; Dr. MALLEPELL, Marc (ETH Zurich) ; Dr. SCHEIDER, Christof (Paul Scherrer Institut) ; Prof. WOKAUN, Alexander (Paul Scherrer Institut)

Oxygen defects in thin oxide films and substrate can have dramatic effects on their transport properties. A well-known system is the dielectric SrTiO3 where a small amount of defects are already sufficient to create electrical conductivity. To understand the oxygen evolution during the growth process of oxides thin film prepared by pulse laser deposition (PLD), it is important to know the role oxygen has in the as-grown film and substrate. To investigate the oxygen diffusion and characterize the interface between film and substrate, Elastic Recoil Detection Analysis (ERDA) and Secondary Ion Mass Spectrometry (SIMS) were employed on theSrTiO3/LaAlO3 system. To investigate the oxygen diffusion in the SrTiO3/LaAlO3 system18O exchanged SrTiO3 and LaAlO3 substrates were prepared prior to the deposition ofLaAlO3. ERDA and SIMS depth profiles show good agreement with respect to the 18Ocontent in the substrate and deposited films. An important finding is that the main oxygen supply for the deposited film originates from the SrTiO3 substrate and significant oxygen diffusion into the LaAlO3 film already takes place at a moderate deposition temperature of $500^{\circ} \mathrm{C}$. This implies that growth-induced defects at theSrTiO3/LaAlO3 interface can already be reduced during the growth via an effective oxygen exchange from the depth of the substrate, but also that the SrTiO3 substrate may be partially reduced at the interface.

\section{6: Optical Emission Studies in Pulsed Nd:YAG Laser Deposition of Diamond-like Carbon}

Presenter : Prof. TOU, Teck Yong

Dr. YAP, Seong Shan (Multimedia University), Mr. NEE, Chen Hon (Multimedia University) ; Mr. SIEW, Wee Ong (Multimedia University) ; Dr. SáFRáN, György (Hungarian Academy of Sciences (MFA)) ; Prof. TOU, Teck Yong (Multimedia University)

This paper presents the time-integrated optical emission spectroscopic (OES) studies of the plasma plume, which is visible, in the pulsed Nd:YAG laser deposition of diamond-like carbon (DLC) in high vacuum. For the graphite ablation, effects of laser wavelength and fluence on line intensities and ionization levels for various carbon plume specie were analyzed. These OES results were correlated to the sp3 content, or the amount of tetrahedrally bonded carbon, in the DLC thin film as deposited from the ablated carbon plume. The sp3 content was estimated indirectly from the Raman spectra and directly by the electron-energy loss spectroscopy (EELS) in the transmission electron microscopy (TEM). The correlation showed a general trend on the kinetic energy of carbon specie which influenced the DLC formation, suggesting sub plantation of carbon specie. 


\section{2: Functional interfaces in oxide hetero structures by atomically controlled pulsed laser deposition (Invited)}

Presenter : Guus RIJNDERS

Prof. RIJNDERS, Guus (MESA- Institute for Nanotechnology / University of Twente)

Complex oxide materials start to play a very important role in electronic devices, since they exhibit a broad range of functional properties, such as high dielectric permittivity, piezoelectricity and ferroelectricity, superconductivity, colossal magnetoresistance and ferromagnetism. Many of these phenomena occur in oxides that are lattice-matched within a few percent of one another. This enables fabrication of heteroepitaxial structures, in which the multiple degrees of freedom can be accessed, making a myriad of devices with novel functionalities possible. However, a completely new class of (nano) devices can be engineered, exploiting the novel functional properties of interfaces in oxides. A real breakthrough in this field is obtained by controlling and tailoring on atomic scale the physical properties at the interfaces. Interfaces modify the electronic correlations, controlling the electronic behavior of the material, and their modification can induce remarkable changes of the collective electronic and magnetic properties. In this contribution, I will focus on the electronic reconstruction at the polar interface between the perovskite oxides SrTiO3-LaAlO3. This model system illustrates the vast variety of observed electronic phenomena that arise from this new type of electron doping, ranging from superconductivity to magnetic scattering. I will address the recent developments as well as future challenges of hetero interfaces between perovskite oxides.

\section{3: Ultrafast laser interaction with group IV materials}

Presenter : Harold HAUGEN.

HAUGEN, H. K. (Department of Engineering Physics and Department of Physics and Astronomy, McMaster University, p

\section{7: Nanocrystal Growth of II-VI wide band gap semiconductors by ultrafast laser ablation and deposition}

Presenter : Marta CASTILLEJO

Dr. CASTILLEJO, Marta (Instituto de Química Física Rocasolano, Spanish National Research Council (CSIC), Serrano 119, 28006 Madrid, Spain), Dr. SANZ, Mikel (Instituto de Química Física Rocasolano, Spanish National Research Council (CSIC), Serrano 119, 28006 Madrid, Spain) ; Dr. DE NALDA, Rebeca (Instituto de Química Física Rocasolano, Spanish National Research Council (CSIC)) ; Ms. LóPEZ-ARIAS, Maria Encina (Instituto de Química Física Rocasolano, Spanish National Research Council (CSIC), Serrano 119, 28006 Madrid, Spain) ; Dr. AMORUSO, Salvatore (Coherentia CNRINFM and Dipartimento di Scienze Fisiche, Università degli Studi di Napoli Federico II, Complesso Universitario di Monte S. Angelo, Via Cintia, I-80126 Napoli (Italy)) ; Dr. AUSANIO, Giovanni (Coherentia CNR-INFM and Dipartimento di Scienze Fisiche, Università degli Studi di Napoli Federico II, Complesso Universitario di Monte S. Angelo, Via Cintia, I80126 Napoli (Italy)) ; Prof. BRUZZESE, Riccardo (Coherentia CNR-INFM and Dipartimento di Scienze Fisiche, Università degli Studi di Napoli Federico II, Complesso Universitario di Monte S. Angelo, Via Cintia, I-80126 Napoli (Italy)) ; Dr. LETTIERI, Stefano (Coherentia CNR-INFM and Dipartimento di Scienze Fisiche, Università degli Studi di Napoli Federico II, Complesso Universitario di Monte S. Angelo, Via Cintia, I-80126 Napoli (Italy)) ; Dr. WANG, Xuan (Lettieri) 
Direct II-VI bandgap semiconductors are important electronic and optoelectronic materials. As transport properties of photons and phonons are largely affected by size and geometry, their fabrication as nanocrystals is receiving increased attention for both fundamental studies and novel applications. We report on the properties of the ablation plume and the characteristics of CdS andZnS grown by ultrafast pulsed laser deposition with pulses of $300 \mathrm{fs}$ at 527 and $263 \mathrm{~nm}$. The ablation plume was characterized by time and space resolved emission spectroscopy and gated imaging. Less than one layer deposits consisting of isolated nanoparticles (NPs) on mica substrates, and NP-assembled films on Si substrates were grown by appropriately changing the number of laser shots. The crystalline quality and composition of the deposits was studied by X-ray diffraction and X-ray photoelectron spectroscopy, while the surface morphology was analyzed by environmental scanning electron microscopy and atomic force microscopy. Photoluminescence of the deposited films was also measured at room temperature. The results are discussed in terms of the composition and expansion dynamics of the semiconductor plasma plume and of the properties of the deposited NPs and nanostructured films. These results also allow investigating the influence of the laser irradiation wavelength on the obtained nanostructures and provide insight on the underlying mechanisms controlling the gas phase and deposition processes.

\section{8: Experimental comparison between intensity and phase detection in grating coupling surface plasmon resonance}

Presenter : Wen-kai KUO

Prof. KUO, Wen-kai (National Formosa University) Mr. CHANG, Chih-hao (National Formosa University

In most practical applications, the surface plasmon resonanc (SPR) can be excited by a p-polarized incident light and two major coupling mechanisms, a prism or a grating coupler. For a fix incident wavelength light wave, the small RI change can be detected by measuring the intensity or phase variations of the reflected light from the metal surface. For a prism coupling SPR scheme, the sensitivity comparisons between intensity and phase detections have been first studied by Nelson and further discussed by others. They claimed that sensitivity of the phase detection can be better than that of the intensity detection by a factor of two orders of magnitude. On the other hand, for the grating coupling SPR scheme, most measurements are based on intensity detection. Though, the grating coupler has a slightly less sensitivity than the prism coupler, the grating coupler can achieve a low-cost and array type sensors. Therefore, the grating coupling SPR scheme still has greatly potential in practical sensor applications. In this paper, intensity and phase detection of SPR using a grating coupling structure has been experimentally compared. A simple experiment set-up to detect the change of the reflective index of air caused by heating is proposed and the result shows that sensitivity of the phase detection is better than that of the intensity by a factor of one order of magnitude. Numerically result also has been obtained by using finite-difference time-domain (FDTD) simulation. 


\section{9: Patterned laser annealing of silicon oxide films}

Presenter : Jürgen IHLEMANN

Mr. RICHTER, Johannes (Laser-Laboratorium Göttingen) Co-authors : Dr. MEINERTZ, Jörg (Laser-Laboratorium Göttingen) ; Dr. IHLEMANN, Jürgen (Laser-Laboratorium Göttingen) Presenter : Dr. IHLEMANN, Jürgen (LaserLaboratorium Göttingen)

UV-absorbing silicon monoxide $(\mathrm{SiOx}, \mathrm{x} \approx 1)$ thin films on fused silica substrates are irradiated by an ArF excimer laser (wavelength $193 \mathrm{~nm}$ ) in the sub-ablation threshold regime. Multipulse irradiation of films with $200 \mathrm{~nm}$ thickness at a fluence of about $100 \mathrm{~mJ} / \mathrm{cm}^{2}$ leads to a significant increase of the UVtransmission indicating the oxidation of $\mathrm{SiOx}$ to $\mathrm{SiO} 2$. The quality of the obtained films after this laser annealing process depends on the oxygen content of the environment. Irradiation in air at atmospheric pressure leads to the formation of sub-micron sized oxide particles on top of the film. Structured illumination is applied to either form areas of the film with changed transmission and refractive index, or for the formation of regular particle patterns with sub-micron periods. These processes can be utilized for the fabrication of phase masks or for various types of surface functionalization.

\section{(Tuesday, 17 August)}

\section{4: Ultrafast Processes and Single Shot Imaging of Clusters with Intense X-Ray Laser Pulses (Invited)}

Presenter : Christoph BOSTEDT

BOSTEDT, Christoph (Linac Coherent Light Sourc/SLAC)

Free electron lasers (FEL) open the door for novel applications ranging from single-shot imaging experiments on nanostructures to ultrafast element specific x-ray spectroscopy. For virtually all experiments with FELs it is of fundamental importance to study how the absorption and ionization properties of nanoscale systems develop in the shortwavelength strong-field domain. We have performed first experiments about x-ray laser pulse - cluster interaction with a combined spectroscopy and imaging approach at both, the FLASH and the LCLS FEL facilities. The new data show qualitatively different processes for (soft) $\mathrm{x}$-ray pulses from the optical strong field regime. Electrons are emitted from the clusters in a direct multistep photoionization process and plasma type absorption is not significant. The investigation of core - shell systems gives evidence for efficient charge redistribution within the cluster. For single-shot imaging experiments at FLASH a MCP based detector system has been developed. The experiments at LCLS have been performed in the CAMP end station, combining large pnCCD imaging detectors and time-of-flight spectrometers in one setup. Modeling the experimental data indicates that the optical constants of the clusters, which are inherently coupled to its electronic structure and thus charge states, change during the femtosecond pulse. The results show that ultra fast scattering is a promising approach to study transient states of matter on a femtosecond time scale. 


\section{10: Study of surface chemical reactions induced by molecules electronically excited in the gas phase}

Presenter : Victor PETRUNIN

Prof. PETRUNIN, Victor (Physics and Chemistry Department, University of Southern Denmark) Dr. PANKIN, Kirill (Physics and Chemistry Department, University of Southern Denmark) ; Mr. NIELSEN, Jacob (Physics and Chemistry Department, University of Southern Denmark) ; Mr. ANDERSEN, Anders (Physics and Chemistry Department, University of Southern Denmark) ; Mr. MADHUKESWARA, Jalgar (Physics and Chemistry Department, University of Southern Denmark)

In experimental approach, based on application of total internal reflection phenomenon on an insulator surface, we localize laser excitation in a narrow boundary gas layer just above the interface. Majority of the excited molecules can reach the surface before the relaxation. Approaching the surface, the electronically excited molecules will naturally experience strong acceleration and alignment. The energy of the excitation is likely to be converted to kinetic motion along the reaction coordinate for chemical adsorption. While recombination reactions in a gas phase require participation of a third body, this strong limitation on the reaction rates is removed upon interaction with a surface. The experiments are performed at high gas pressures, so that dense fluxes of the excited reagents can be readily produced. Products of chemical adsorption and/or chemical reactions induced within adsorbates are aggregated on the surface and observed by light scattering. Results of evanescent wave illumination are compared with those for laser beam propagating through the interface. We will demonstrate how pressure and spectral dependencies of the chemical outcomes, polarization of the light and interference of two laser beams inducing the reaction can be used to distinguish the new process from reactions induced by photoexcitation within adsorbed molecules and/or gas phase photolysis. High quantum yields of chemical transformations are observed when $\mathrm{NO}$ and $\mathrm{SO} 2$ molecules are photoexcited.

\section{11: Phase transition in carbon based material induced by intense femtosecond $x$-ray pulse}

Presenter : Dr. Jérôme GAUDIN

Dr. GAUDIN, Jérôme (European XFEL GmbH, Hamburg - Germany), BOSTEDT, C. (SLAC National Accelerator Laboratory, Menlo Park-USA) ; BOZEK, J. (SLAC National Accelerator Laboratory,Menlo Park- USA) ; CHALUPSKY, J. (Institute of Physics, Academy of Sciences of the Czech Republic, Prague - Czech Republic) ; HAJKOVA, V. (Institute of Physics, Academy of Sciences of the Czech Republic, Prague - Czech Republic) ; HAU-RIEGE, S. (Lawrence Livermore National Laboratory, Livermore - USA) ; JESCHKE, H.o. (Goethe University of Frankfurt am Main - Germany) ; JUHA, L. (Institute of Physics, Academy of Sciences of the Czech Republic, Prague - Czech Republic) ; KRZYWINSKI, J. (SLAC National Accelerator Laboratory, Menlo Park- USA) ; MOELLER, S. (SLAC National Accelerator Laboratory, Menlo ParkUSA) ; ORTIZ, C. (Goethe University of Frankfurt am Main - Germany) ; SOBIERAJSKI, R. (Institute of Physics PAS, Warsaw - Poland) ; SINN, H. (European XFEL GmbH, Hamburg Germany) ; ZIAJA-MOTYKA, B. (CFEL, Hamburg Germany) ; TSCHENTSCHER, Th. (European XFEL GmbH, Hamburg - Germany) ; TIEDTKE, K. (HASYLAB / DESY, Hamburg Germany)

Our contribution will address the light induced phase transition in carbon based material. In carbon the interaction are enriched by the 3 possible hybridizations: $\mathrm{sp} 1, \mathrm{sp}^{2}$ and $\mathrm{sp}^{3}$ leading to different bonding networks from amorphous phase and nanostructures to single crystal. One particular phase transition of interest is the graphitization process, i.e. the transition from $\mathrm{sp}^{3}$ to $\mathrm{sp}^{2}$ hybridization. It has been shown that this process can be triggered via a non-thermal pathway by irradiation with an ultrashort light pulses in the visible wavelength range. Extending the study of such non-thermal 
transition to the x-ray photon energy range is now possible with the advent of FEL light sources.

We will present the results of recent experiments done at the FLASH and LCLS. Sp3-bonded carbon, single crystal diamond and amorphous carbon, were irradiated by intense femtosecond x-ray pulses. The results allow determining the fluence threshold for graphitization process for 91, 177 and $830 \mathrm{eV}$. Furthermore, by using different surface investigation methods, we characterized the irradiated samples. In both case nanocrystallites of graphite are formed but depending on the initial structure of the material, two different path ways seems to lead to this same final structure. The possible non-thermal origin of this phase transition will be discussed and compared to preliminary results obtained from theory. The model also provides an insight of the dynamics on the femtosecond time scale.

\section{5: Ultrafast Delocalization of Hydrogen Atoms in Hydrocarbon Molecules in Intense Laser Fields (Invited)}

Presenter : YAMANOUCHI, Kaoru

Prof. YAMANOUCHI, Kaoru (The University of Tokyo)

In the present talk, we introduce our recent studies on the hydrogen migration processes in allene by coincidence momentum imaging (CMI) [5,6]. In order to examine how far and how fast ultrafast hydrogen migration proceeds within a molecule in intense laser fields, we chose allene $(\mathrm{CH} 2=\mathrm{C}=\mathrm{CH} 2)$, and investigated its two-body and three-body Coulomb explosion processes by the CMI method. From the CMI maps, two types of two-body Coulomb explosion pathways from doubly charged parent ion, $\mathrm{C} 3 \mathrm{H} 42+$, were securely identified, that is, the fragmentation pathways in which one of the two $\mathrm{C}-\mathrm{C}$ bonds is broken, $\mathrm{C} 3 \mathrm{H} 42+\rightarrow \mathrm{CHm}++\mathrm{C} 2 \mathrm{H} 4-\mathrm{m}+(\mathrm{m}=1-3),(1)$ and the fragmentation pathways in which $\mathrm{Hm}+$ is ejected, $3 \mathrm{H} 42+\rightarrow \mathrm{Hn}++\mathrm{C} 3 \mathrm{H} 4-\mathrm{n}+(\mathrm{n}=1-3)$. (2) From the CMI maps, two three-body Coulomb explosion pathways from triply charged allene, $\mathrm{C} 3 \mathrm{H} 43+$, that is, $\mathrm{C} 3 \mathrm{H} 43+\rightarrow \mathrm{H}++\mathrm{CH}++$ $\mathrm{C} 2 \mathrm{H} 2+$, (3) $\mathrm{C} 3 \mathrm{H} 43+\rightarrow \mathrm{H}++\mathrm{CH} 2++\mathrm{C} 2 \mathrm{H}+$, (4) were also identified. The formation of $\mathrm{CH} 3+$ and $\mathrm{C} 2 \mathrm{H} 3+$ in Eq. (1) and the formation of $\mathrm{H} 3+$ in Eq. (2) can be regarded as direct evidence that the hydrogen atom migration proceeds prior to the Coulomb explosion. Furthermore, the route of a proton migrating from one end to the other end of an allene molecule was mapped from the momentum correlation maps as well as by the geometrical structure of $\mathrm{C} 3 \mathrm{H} 43+$ constructed from the observed momentum vectors of the fragment ion.

\section{6: Atomistic simulations of laser interactions with metals: Melting of nanocrystalline films and generation of crystal defects (Invited)}

Presenter : Leonid ZHIGILEI

Prof. ZHIGILEI, Leonid (University of Virginia), Dr. LIN, Zhibin (Colorado School of Mines) ; Ms. ABDUL KARIM, Eaman (University of Virginia) ; Mr. WU, Chengping (University of Virginia)

Atomic-level computer modeling has the ability to provide detailed information on the complex processes induced by short pulse laser irradiation of metal targets and can assist in the advancement of 
laser-driven applications. Recent results obtained in simulations of laser interactions with metal targets are reviewed in the presentation with a particular focus on the laser-induced generation of crystal defects and therole of the microstructure of the target on the material response to the fast laser energy deposition. In particular, to address the role of the microstructure of the irradiated target on laserinduced phase transformations, the results of the simulations of short pulse laser melting of single crystal films and bulk targets are compared with computational predictions obtained for nanocrystalline Au films. The effect of nanocrystalline structure on the melting process is investigated for abroad range of laser fluences. At low laser fluences, close to the threshold for the complete melting of the film, the initiation of melting at grain boundaries is found to steer the melting process along the path where the melting continues below the equilibrium melting temperature of the material and the crystalline regions shrink and disappear under conditions of substantial undercooling. The unusual melting behavior of nanocrystalline films is explained based on thermodynamic analysis of the stability of small crystalline clusters surrounded by under cooled liquid.

\section{12: The role of anisotropic excitation in self-organized nanostructure formation upon femtosecond laser ablation}

Presenter :. Juergen REIF

Prof. REIF, Juergen (Brandenburgische Technische Universitaet (BTU) Cottbus) VARLAMOVA, Olga (BTU Cottbus and Cottbus JointLab) ; Dr. VARLAMOV, Sergej (BTU Cottbus) ; Prof. BESTEHORN, Michael (BTU Cottbus) ; Prof.

MIYAZAKI, Kenzo (IAE Kyoto University) ; Dr. MIYAJI, Godai (IAE - Kyoto University)

Self-organized nanostructures (ripples, LIPSS) developing at the surface of all types of materials upon femtosecond laser ablation exhibit, generally, a strong influence of the laser polarization on their orientation and shape. This dependence is very surprising, taking into account that the laser electric field is present only at the first step of electronic excitation. Already during excitation transfer to the lattice and, even more, during particle emission (ablation) and subsequent structure formation, the laser field is NOT present. We present a theoretical model and corresponding simulations indicating a possible explanation for this phenomenon. Our model shows that a spatial anisotropy of the excitation, even due to an asymmetric beam profile, may favour a directional anisotropy in the pattern generation. Much stronger will be the anisotropy induced by polarization dependent coupling to plasmons/polaritons created at the irradiated surface. Since this anisotropy is not sensitive to short range dissipation, it persists at much longer time than the femtosecond excitation Numerical simulation of the consequences of this model yields typical patterns which will be compared to experimental observations under appropriate conditions.

\section{13: The effect of laser wavelength on heating of the ablated plume}

Presenter : Zygmunt SZYMANSKI,

Prof. SZYMANSKI, Zygmunt (Institute of Fundamental Technological Research) Co-authors : Dr. HOFFMAN, Jacek (Institute of Fundamental Technological Research) ; Dr. MOSCICKI, Tomasz (Institute of Fundamental Technological Research)

During laser ablation the material evaporated from the target forms a thin layer of very dense gas, consisting of electrons, ions and neutrals. This plasma plume absorbs energy from the laser beam and 
its temperature and pressure grow. Main heating mechanisms are inverse Bremsstrahlung and photoionization and both mechanisms depend on the laser wavelength. While electron-ion inverse Bremsstrahlung is easy to calculate since Coulomb cross section is well known, the electron-atom inverse Bremsstrahlung and photoionization require knowledge of electron-atom scattering and photoionization cross sections. In this paper laser heating of the plume evaporated during ablation of graphite is studied. Graphite was chosen because its ablation is used to obtain a wide variety of carbon related materials, such as diamond-like carbon (DLC), fullerene carbon molecules, and carbon nanotubes. Moreover, the relevant cross sections for carbon atoms and ions are available, which enables the analysis of the photon absorption. Graphite target irradiation was performed using an $\mathrm{Nd}$ :YAG laser which operated at a wavelength of 1064, 533, $355 \mathrm{~nm}$ with a pulse energy of $\sim 400 \mathrm{~mJ}$ and $10 \mathrm{~ns}$ pulse duration. The laser fluence was $15 \mathrm{~J} \mathrm{~cm}-2$. Plasma parameters in early phase of expansion (up to $100 \mathrm{~ns}$ from the laser pulse) were studied as a function of a distance from the target. Considerable differences of the order of ten thousand Kelvins were found in the case of different wavelengths

\section{14: Dynamics of fast phase transitions in metals during pulsed laser action}

Presenter :. Alexander MAZHUKIN

Dr. MAZHUKIN, Alexander (Keldysh Applied Mathematics Institute of Russian Academy of Sciences) Prof. MAZHUKIN, Vladimir (Keldysh Applied Mathematics Institute of Russian Academy of Sciences) ; Dr. DEMIN, Michael (Keldysh Applied Mathematics Institute of Russian Academy of Sciences)

Short and ultrashort laser action on aluminum, copper and gold is considered. Pulsed nano- and picosecond action is characterized by non-equilibrium heating of metals described within the framework of two-temperature approximation. Rapid heating is caused by the appearance of phase transitions of the first order which velocity increases as the pulse duration drops and intensity rises. Mathematical modeling with explicit interphase front tracking allowed determining that fast phase transformations cause high degree of non-equilibrium of the processes which manifests itself as highly overheated metastable regions in solid and liquid phases. The magnitude of overheating depends on the pulse duration. Typical overheating is tens and hundreds of degrees in the nanosecond range and thousands of degrees in the picosecond range.

Work is supported by RFFI (projects N 10-07-00246-a, N 10-07-00246-ofi-m).

\section{7: Phonon-assisted nanophotonic fabrication and its application (Invited)}

Presenter : Motoichi OHTSU

Motoichi OHTSU, (The University of Tokyo)

This presentation reviews novel nanophotonic fabrication techniques based on a phononassisted process triggered by optical-near-field interactions. This process represents qualitative innovation in photochemical vapor deposition and photolithography, suggesting that large, expensive ultraviolet light sources will no longer be required, although they are indispensable for conventional adiabatic photochemical vapor deposition, photolithography, and photochemical etching. It also suggests that phonon-assisted photochemical vapor deposition can even dissociate optically inactive molecules (i.e., inactive to propagating light), which is advantageous for environmental protection 
because most optically inactive molecules are chemically stable and harmless. For example, optically inactive $\mathrm{Zn}(\mathrm{acac}) 2$ molecules have been dissociated to deposit nanometric $\mathrm{Zn}$ particles. In addition, in the case of phonon-assisted photolithography, an optically inactive resist film for electron-beam lithography has been used to fabricate fine patterns. A prototype commercial lithography system has been produced in collaboration with industry, and has been used for fabricating diffraction gratings and Fresnel zone plates for soft X-rays. Furthermore, phonon-assisted photochemical etching has realized an ultra-flat glass surface with a roughness as low as $0.13 \mathrm{~nm}$ without using any photomasks.

\section{15: Precipitation of ferromagnetic nanoparticles inside transparent glass by femtosecond laser}

Presenter : Dr. Seisuke NAKASHIMA

Dr. NAKASHIMA, Seisuke (RIKEN), Prof. SUGIOKA, Koji (RIKEN) ; Prof. MIDORIKAWA, Katsumi (RIKEN)

Metallic nanoparticles (NPs) can be space-selectively precipitated inside transparent materials by femtosecond (fs) laser irradiation. In most cases, they are Au and Ag ortheir alloys for applications to 3D marking and so on. However, there is no report on localized dispersion of magnetic functional NPs inside materials, which can be applied to many devices, such as 3D high density magnetic recording devices and magneto-optical components integrated with 3D optical waveguide devices. In this paper, we report a photo-induced precipitation of magnetic NPs inside transparent glass materials. Fe-ion doped silicate glasses were prepared using glass melting method. The glass sample was irradiated with fs-laser $(\lambda=775 \mathrm{~nm})$ followed by annealing. The absorption spectrum for irradiated and annealed glass exhibited a peak at around $400 \mathrm{~nm}$, which corresponds to local surface plasmon absorption of precipitated NPs. In contrast to diamagnetic M-H curve measured for as-prepared glass, a erromagnetic behavior superimposed on the diamagnetic component due to glass matrix was observed in the M-H curve for irradiated and annealed glass. Using TEM, we observed NPs dispersed in the irradiated part of glass sample. It is speculated that the ferromagnetic high magnetization is attributable to metallic Fe NPs locally precipitated by the fs laser irradiation and post anneal. Meanwhile, the annealed glass without irradiation with fs laser remained unchanged magnetically as well as optically.

\section{16: Preparation and formation mechanism of amorphous Si quantum dots by laser ablation of silicon in water}

Presenter : X. Y.CHEN

Dr. CHEN, X. Y. (Department of Materias Science and Engineering, Nanjing University, Nanjing 210093) Co-authors : Dr. WU, Di (Department of Materias Science and Engineering, Nanjing University, Nanjing 210093)

In recent years it was found that laser ablation of a solid target immersed in liquid can lead to formation of nanoparticles of the target material in the liquid, and now it is becoming a promising technique for preparation of nanoparticles. However, its mechanisms are still not understood very well. So it is still difficult to control size and its distribution of the resulted particles. In this paper, we will show that, in laser ablation of silicon in water, a new parameter, namely the distance between the target and the surface of the liquid, plays an important role in determining the particle size and its distribution. With 
the optimized distance, highly homogeneous silicon quantum dots with size of $\sim 3 \mathrm{~nm}$ were obtained. The underlying mechanism is proposed to be that the distance can affect the expansion dynamics of the silicon plasma bubble formed by laser ablation in water and so the cooling and condensation processes of the plasma bubble. We believe that this mechanism provides a new way to control the nanoparticles prepared with this method.

\section{17: Dynamics of Exciton-Plasmon Coupling in ZnO-MgO-Metal Heterostructures}

Richard HAGLUND

Prof. HAGLUND, Richard (Vanderbilt University), Mr. LAWRIE, Benjamin (Vanderbilt University) ; Prof. MU, Richard (Fisk University and Vanderbilt University)

Exciton-plasmon coupling models the fundamental interaction between one-particle and collective excitations in condensed matter. We are investigating the coupling between the band-edge exciton and visible luminescence centers and plasmonic nanostructures using time-resolved photoluminescence (PL) and both broad-band and frequency-degenerate pump-probe (PP) spectroscopy. The model system is a thin-film heterostructure comprising a $\mathrm{ZnO}$ thin film or quantum well separated from a plasmonic metal layer by a $\mathrm{MgO}$ spacer film of varying thickness. The plasmonic layer may be either nanodisc arrays or a rough film of $\mathrm{Au}, \mathrm{Ag}$, or $\mathrm{Al}$; the localized surface plasmon resonance (LSPR) dominates the behavior of the former, while the surface plasmon polariton (SPP) is characteristic of the latter. Au and Ag SPPs demonstrate a Purcell-like enhancement of the band-edge exciton PL, while the visible luminescence center, arising from intrinsic defects, couples more strongly to the Auand Ag LSPR and shows a distance dependence typical of the coupling between a single dipole and an array of dipole antennas. The peak PL enhancement redshifts from the $\mathrm{ZnO}$ band-edge to the SPP resonance with increasing $\mathrm{MgO}$ thickness, indicating a mechanism not consistent with the conventional Purcell model. The Al LSPR varies from $360 \mathrm{~nm}$ to $550 \mathrm{~nm}$ with increasing nanodisc diameter and should therefore couple in principle to both the exciton and the defect luminescence centers depending on the nanoparticle size.

\section{8: Nanoparticles produced by laser ablation (Invited)}

Presenter. Paolo M. OSSI

Prof. OSSI, Paolo M. (Politecnico di Milano), Prof. NERI, Fortunato (Dipartimento di Fisica della Materia e Ingegneria Elettronica, Università di Messina) ; Dr. SANTO, Nadia (Centro Interdipartimentale Microscopia Avanzata, Università degli Studi di Milano) ; Dr. TRUSSO, Sebastiano (CNR, Istituto per i Processi Chimico-Fisici, Messina)

The deposition of noble metal Ag and Au nanostructured thin films by pulsed laser ablation in inert gas is described. The films consist of nanoparticles (NPs) whose average size, as measured by TEM, sensitively depends on ambient gas nature and pressure. Fast imaging of the plasma allows deducing the propagation regime of the ablated plume and its velocity. The latter data were used together with the measured average ablated mass per pulse as input parameters in a model to estimate the average size of NPs grown in the expanding plasma. For samples grown in different conditions TEM pictures indicate a reduced dispersion of particle size over narrow size ranges. Thus a fine tuning of NP size is achievable by a strict control of synthesis parameters. The production of SERS active substrates whose optical properties (surface plasmon resonance) and SERS activity critically depend on 
NP size, shape and structure is discussed.

\section{18: Novel processing for the oxide films by photo reaction of nano-particles}

Presenter. Tetsuo TSUCHIYA,

Dr. TSUCHIYA, Tetsuo (National Institute of Advanced Industrial Science and Technology(AIST)), Dr. NAKAJIMA, Tomohiko (AIST) ; Dr. KUMAGAI, Toshiya (AIST)

Metal oxide materials have created considerable interest in recent years for their potential application to electric device. Among the unsolved problems of the processing, a development of the low temperature and patterning process has been increasingly recognized. To overcome these problems, we have developed a new thin film process for the oxide materials on organic substrate using photo reaction of nano-particles method (PRNP)[1]. In this paper, we demonstrate the preparation of transparent conducting thin film and phosphor film films by the photo- induced chemical solution process, and their properties of the obtained film. To prepare a polycrystalline oxide thin film on glass or PET substrates, we have developed the photo reaction of the nano-particles (PRNP) using an excimer lamp and laser at room temperature without vacuum. By using the combined two-step irradiation and onestep irradiation process, ITO and phosphor thin film were prepared on glass and PET substrates at room temperature. The electrical resistivity, carrier concentration, Hall mobility and transmittance at $550 \mathrm{~nm}$ of the ITO film on glass were $5.94 \times 10^{-4} \Omega \mathrm{cm}, 1.05 \times 1021 \mathrm{~cm}-3$ to and $9.99 \mathrm{~cm}-2 \mathrm{~V}-1 \mathrm{~s}-1$ and $81 \%$, respectively. By using PRNP, the compact microstructure ITO film was found to be formed. Also, we have successfully obtained phosphor film using nano-particles. Thus, the PRNP method is useful for the oxide film on organic substrate. [1] T. Tsuchiya et al. Appl. Phys. A (in press))

\section{19: Self-organised nanostructures on metal surfaces produced by femtosecond laser ablation}

Presenter : Dr. Evgeny GUREVICH

Dr. GUREVICH, Evgeny (ISAS, Institut fur Analytische Wissenschaften)

Self-organised patterns are known in different systems driven out of equilibrium, like chemical reactions, ecological systems, granular media and in many others. Here the self-assembled structures observed on the surface of craters, left on metal surfaces by means of femtosecond laser ablation are discussed. In the course of femtosecond laser ablation the sample surface temperature rises above the melting point within several picoseconds and the melt layer becomes overheated. Periodic stripes, convection-like cells and isolates structures appear in the melt layer. The transient pattern stays frozen as the melt solidifies on a nanosecond time scale. This allows characterisation of the transient patterns by means of scanning electron microscopy. Some properties of the self-organised patterns can be controlled by the laser beam parameters. Physical processes leading to the appearance of the selfassembled structures and the pattern properties are not clear yet. Possible mechanisms as well as control strategies of the pattern formation are discussed. 


\section{20: Selective ablation of Xe on silicon surfaces: MD simulation and experimental laser patterning}

Presenter : Ori STEIN

Mr. STEIN, Ori (Hebrew University) ; Prof. ASSCHER, Micha (Hebrew University)

Laser induced ablation of multilayer $\mathrm{Xe}$ on $\mathrm{Si}$ has been studied employing molecular dynamics (MD) simulations. $5 \mathrm{nsec}$ long laser pulse at $\lambda=337 \mathrm{~nm}$ was applied to a Xe slab at thicknesses of 16,32 and 40ML (7744, 15488, 19360 atoms, respectively) adsorbed on top of an 8 layers 5408 atoms Si slab. Evaporative and explosive ablation threshold were identified at absorbed laser power of 12 and $16 \mathrm{MW} / \mathrm{cm} 2$ which corresponds to surface temperature rise of 500 and $658 \mathrm{~K}$, respectively. Selective ablation was studied, where only a fraction of the lateral dimension of the computation cell was actually ablated. Extremely strong lateral dissipation among the Xe layers, has led the ablation threshold to shift to higher laser power as the fraction of heated area shrinks. Heated fraction (HF) less than $10 \%$ results in practically no ablation at laser power below substrate damage threshold. The MD studies were assessed and verified by experimental laser ablation measurements. A 10nsec Nd:YAG laser pulse operating at $\lambda=532 \mathrm{~nm}$ was employed. It was found that for 80 and $160 \mathrm{ML}$ Xe layer thickness, full ablation was reached at laser power of 6.9 and $8.4 \mathrm{MW} / \mathrm{cm} 2$ which corresponds to surface temperature rise of 180 and $220 \mathrm{~K}$ respectively. Line-edge profile resulting from fractional laser induce desorption coverage grating formation followed by metallic lift-off experiments were compared to the MD simulations of selective ablation, revealing a remarkable similarity.

\section{(Wednesday, 18 August)}

\section{9: Laser interactions with organic MALDI matrices (Invited)}

Presenter: Klaus Dreisewerd

Klaus Dreisewerd, University of Münster, Germany

\section{21: Investigating the fragmentation of C60 by Matrix Assisted Pulsed Laser Evaporation (MAPLE)}

Presenter : Stela CANULESCU

Dr. CANULESCU, Stela (Department of Photonics Engineering, DTU Fotonik, Technical University of Denmark) ;Dr. SCHOU, Jørgen (Department of Photonics Engineering, DTU Fotonik, Technical University of Denmark), Dr. NIELSEN, Søren Fæster (Materials Research Division, Risø National Laboratory for Sustainable Energy, Technical University of Denmark)

We study possible fragmentation effects that can occur during the transfer of $\mathrm{C} 60$ molecules in vacuum by matrix assisted pulsed laser evaporation (MAPLE). The MAPLE studies are performed on a frozen homogeneous dilute solution of C60 in anisole (typically $0.67 \mathrm{wt} . \%$ ), and over a broad range of laser fluences, from $0.3 \mathrm{Jcm}-2$ up to $2 \mathrm{Jcm}-2$. The processing of the $\mathrm{C} 60$ molecules into thin films of C60, 
and for other fullerenes can be of large interest not only for fundamental studies, but also for organic solar cells applications.For a given MAPLE matrix, possible fragmentation effects were monitored by measuring the deposition rate/pulse and determining the fragmentation rate with matrix assisted laser desorption ionization (MALDI). Analyses of high-resolution SEM images reveal that the surface of the MAPLE films consists of a high density area of clusters/droplets. These round shaped features are probably caused by ejection of large liquid droplets of C60 suspended in solvent upon laser irradiation, followed by the evaporation of the solvent when reaching the substrate. MALDI analyses of the C60 layers deposited onto $\mathrm{Si}$ wafers have shown that a significant fraction of fullerenes remain intact upon MAPLE transfer.

\section{22: Mechanistic evaluation of 213 nm pulsed laser cornea ablation}

Presenter : Wolfgang Prof. KAUTEK

Prof. KAUTEK, Wolfgang (University of Vienna, Department of Physical Chemistry), Dr. BATISHCHE, S. (National Academy of Sciences of the Republic of Belarus, Institute of Physics) ; Mr. BUSHUK, S. (National Academy of Sciences of the Republic of Belarus, Institute of Physics) ; Mr. KOUZMOUK, A. (National Academy of Sciences of the Republic of Belarus, Institute of Physics) ; Mr. SAVITCH, A. (National Academy of Sciences of the Republic of Belarus, Institute of Physics) ; Mr. TATUR, H. (National Academy of Sciences of the Republic of Belarus, Institute of Physics) ; Prof. GRABNER, G. (Paracelsus Medical University (PMU), University Eye Clinic)

Far-ultraviolet nanosecond pulse Nd:YAG laser processing at $213 \mathrm{~nm}$ promises various advantages for ophthalmologic laser applications involving the reshaping of corneas [1] in respect to gas excimer lasers (e.g. ArF, $193 \mathrm{~nm}$ ) [2], and even femtosecond lasers [3, 4]. Deployment of solid state lasers would eliminate the hazards of a gas laser and would reduce its size and footprint. The insitu investigation of the absorptive and ablation behaviour of fresh corneas yielded new insight into the mechanism of $213 \mathrm{~nm}$ radiation cornea ablation. The involvement of phenomena such as microbubbles of saturated water steam around hot cornea collagen filaments, overheated water steam as real gas with pressures of more than 200 bar, temperatures of more than $300 \mathrm{oC}$, and the generation of low temperature plasmas at higher fluences were detected. Another important issue supporting the precision-control in laser surgery was the degree of corneal hydration linked to ablation rates.

1. Q. Ren, G. Simon, J.M. Legeais, J.M. Parel, W. Culbertson, J. Shen, Y. Takesue, and M. Savoldelli, Ophthalmology 101 (1994) 883.

2. W. Husinsky, S. Mitterer, J. Altmann, G. Grabner, I. Baumgartner, F. Skorpik, and T. Asenbauer, Refract. Surg. 7 (1991) 385.

3. W. Kautek, S. Mitterer, J. Krüger, W. Husinsky, and G. Grabner, Appl. Phys. A58 (1994) 513.

4. J. Krüger and W. Kautek, in Advances in Polymer Science, Vol. 168, (Ed.) T. Lippert, Springer-Verlag Berlin Heidelberg, 2004, p. 247. 


\section{10: Production of films of organic materials for biomedical applications by laser-assisted methods (Invited)}

Presenter : Ion N MIHAILESCU

Prof. MIHAILESCU, Ion N. (INFLPR)

We review the deposition by MAPLE of thin films of various biomaterials. We present comparative results on thin films of pullulan biopolymer obtained by PLD and MAPLE. We demonstrated that only MAPLE is appropriate for transfer with high structural fidelity. We analyze the transfer of novel PMMA-bioglass composites to uniform thin layers. The cells cultivated on PMMA-bioglass layers cover almost entirely the structures. The nanohybrid structures consisting of hydroxyapatite (HA) and sodium maleate-vinyl acetate copolymer (MP) were investigated for bone implantology applications. The thin films of Alendronate-HA composite nanocrystals with increasing bisphosphonate content were synthesized for osteoporosis treatment. In the presence of alendronate, MG63 cells displayed a normal morphology, increased proliferation and higher values of differentiation parameters. In contrast, osteoclasts showed significantly reduced proliferation and increased level of Caspase 3. Normal cell morphology and optimal spread with differentiation potential were evidenced when human osteoblast precursor cells were cultivated on ECM protein modified surfaces. We immobilized urease in form of thin films. The kinetic analyses indicated that the laser immobilized enzyme is active in breaking down urea. We conclude that the deposited thin films and structures are identical to the starting material, preserving their chemical structure and very likely their functionality and biologic activity.

\section{11: Double pulse ultrafast laser ablation of metals}

Presenter : Salvator AMORUSO

Dr. AMORUSO, Salvatore (Dipartimento di Scienze Fisiche and CNR-SPIN, Università degli Studi di Napoli Federico II, Complesso Universitario di Monte S. Angelo, Via Cintia, I-80126 Napoli (Italy)) Prof. BRUZZESE, Riccardo (Dipartimento di Scienze Fisiche and CNR-SPIN, Università degli Studi di Napoli Federico II, Complesso Universitario di Monte S. Angelo, Via Cintia, I-80126 Napoli (Italy)) ; Dr. WANG, Xuan (CNR-SPIN, Complesso Universitario di Monte S. Angelo, Via Cintia, I-80126 Napoli (Italy)) ; Prof. LUNNEY, James (School of Physics, Trinity College Dublin, Dublin 2, Ireland) ; Dr. DONNELLY, Tony (School of Physics, Trinity College Dublin, Dublin 2, Ireland) ; Mr. O’CONNELL, Gearoid (School of Physics, Trinity College Dublin, Dublin 2, Ireland)

Ultrafast laser ablation (ULA) is a unique material processing technique covering several application areas, such as nanoparticle (NP) generation, thin film growth, material processing and LIBS. This has stimulated interest in strategies aiming to modify or control the properties of this process. The use of a pair of laser pulses(double pulse, DP), with controlled temporal separation, seems to offer aninteresting route to changing the ULA process through the effects brought about by the interaction of the delayed pulse with a modified target surface or with the different components of the nascent ablation material. We report on DP ULA of metallic targets $(\mathrm{Ni}$, $\mathrm{Cu})$ with DP ( $\sim 300 \mathrm{fs}, 527 \mathrm{~nm})$, separated by $\sim 1-1000 \mathrm{ps}$. The properties of the ablated species (ions, atoms and NPs) were studied using ion probe, optical emission spectroscopy and fast imaging. The ablation efficiency was measured by analyzing depth profiles of craters produced on a stationary target with a number of laser pulses. Our experimental findings indicate that DP irradiation 
strongly influences thecomposition of ablated material and the ablation efficiency [1,2]. The second pulse interacts with the vapour/plasma initially formed by the first pulse, and in turn affects the overall ULA process.

\section{23: Pulsed laser deposition of La0.6Ca0.4MnO3: ion dynamics and thin film properties}

Presenter : Dr. Thomas Lippert

Dr. LIPPERT, Lippert Thomas (Paul Scherrer Institut), ESPOSITO, Martin (Paul Scherrer Institut) ; Dr. SCHEIDER, Christof (Paul Scherrer Institut) ; Prof. WOKAUN, Alexander (Paul Scherrer Institut)

The growth of manganite thin films by PLD is challenging due to the manganite's composition dependent rich phase diagram. To grow manganite thin films with the desired properties, it is therefore important to understand the chemical processes in the plasma plume during the ablation. In this study Langmuir probe, emission spectroscopy, quartz microbalance and quadrupole mass spectrometry were employed to fully characterize the plasma composition and their energy distribution in the plume as function of the background pressure and background gas, i.e. in vacuum and $\mathrm{N} 2 \mathrm{O}$ and $\mathrm{O} 2$ background gases. After the film deposition the respective composition was analyzed in-situ by secondary ion mass spectrometry (SIMS) to attempt a correlation of the plasma composition with the film composition. For vacuum ablation, the plume consists mainly of metal atoms and ions $(\mathrm{La}, \mathrm{Ca}, \mathrm{Mn}, \mathrm{O}, \mathrm{LaO})$ as well as multiatomic species such as $\mathrm{LaMnO}+$. The $\mathrm{LaO}+$ diatomic species are by far the most intense diatomic species in the plume, while $\mathrm{CaO}$ and $\mathrm{MnO}$ are only detected in minor quantities. The interaction of the more reactive $\mathrm{N} 2 \mathrm{O}$ gas with the ablation plume leads to a significant increase in plume reactivity even compared to the $\mathrm{O} 2$ atmosphere. This enhanced reactivity is important to grow manganite thin films in order to achieve the desired oxygen content. A comparison between the optimized plasma conditions, film composition, and structural as well as transport data will be presented.

\section{24: Electromagnetic control of plume expansion in laser ablation}

Presenter. James G. LUNNEY

Prof. LUNNEY, James G. (Trinity College Dublin), Ms. PAGANO, Clelia (Trinity College Dublin)

For some applications of laser ablation it would be beneficial to control the expansion of the ablation plume. When the plume is significantly ionised, the moving plasma will interact with a static magnetic field. There are several reports of experiments where has been demonstrated that a magnetic field can strongly influence the flow of laser produced plasma [1,2]. We report the results of two experiments on using a static magnetic field to control the expansion of a laser ablation plasma. In the first the expansion of copper laser produced plasma along a magnetic field was investigated with the aim of confining the lateral expansion of the plasma. In the second a plasma lens, which uses a combination of magnetic and electric fields, was used to concentrate the plasma flow. Time-resolved optical imaging, time- and space-resolved optical emission spectroscopy, time-of-flight Langmuir ion probe measurements and thin film deposition measurements were used to study the plasma dynamics. For both devices we observed substantial confinement of the plasma expansion and a large increase in the deposition rate. 


\section{25: Laser-induced breakdown spectroscopy for the analysis of strontium and uranium in glass matrix}

Presenter: E. C. Jung

Dr. JUNG, E.c. (Korea Atomic Energy Research Institute) Co-authors : Mr. LEE, D.h. (KAIST) ; Prof. YUN, J.-i. (KAIST) ; Dr. KIM, J.g. (Korea Atomic Energy Research Institute) ; Dr. YEON, J.w. (Korea Atomic Energy Research Institute) ; Dr. SONG, K. (Korea Atomic Energy Research Institute)

Laser-induced breakdown spectroscopy has been applied to quantify trace elements contained in glass matrix. The measurements were performed using a second and third harmonics of $\mathrm{Nd}: \mathrm{YAG}$ laser beam at $532 \mathrm{~nm}$ and $355 \mathrm{~nm}$, respectively, for the generation of a laser-induced plasma on a glass surface. The spectral lines of the major and trace constituentss were analyzed simultaneously in the wavelength range from 200 to $780 \mathrm{~nm}$ by an echelle spectrometer. The emission intensities increased about 10 times for the UV laser beam at $355 \mathrm{~nm}$ due to an increase of ablation rate. Thus, the elementselective limits of detection (LODs) for strontium ( $\mathrm{Sr}$ ) and uranium (U) were determined with the UV wavelength. In order to obtain calibration curves for concentration measurement of $\mathrm{Sr}$ and $\mathrm{U}$, the emission intensities at $460.733 \mathrm{~nm}$ and $358.488 \mathrm{~nm}$ were measured, respectively, for several samples having different concentrations. The LOD for $\mathrm{U}$ is about a few hundreds times higher than that of Sr. Possible reasons for the difference in the LODs will be discussed based on the measurement of the plasma temperature and differential thermal analysis for several glass samples

\section{26: Surface and bulk structuration of solid and liquid dielectrics}

Presenter : Candice Mézel

Ms. Mézel, Candice (CELIA (Centre Lasers Intenses et Applications)), Dr. HALLO, Ludovic (CELIA) ; Ms. SOUQUET, Agnès (INSERM) ; Dr. BOURGEADE, Antoine (CEA-CESTA) ; GUILLEMOT, Fabien (INSERM)

Ultrafast laser interaction with dielectrics may produce very high energy density hotspots in the bulk as well as at the material surface, depending on focusingconditions. This high energy density is due to ionization of the initially transparent solid by some transfers from the femtosecond laser energy to electrons of the valence band, which finally produces conduction band electrons. The detailed processes involved are free electrons generation and heating by Joule effects, which occurs while electromagnetic wave propagates into the material. A self-consistent model including solution of 3D Maxwell equations, a rate equation for free electrons and several ionization models has been utilized for calculations of laser energy deposition in the bulk or near dielectric boundaries. Heating of the matter is performed in a time shorter than acoustic time, i.e. before any hydrodynamic process. Then nanostructuration of the matter begins due to subsequent hydrodynamic processes. Some applications will be presented: the bulk structuration by formation of submicrometric cavities, and the surface structuration by jet formation similar to the LIFT (Laser Induced Forward Transfer) process in liquid. Surface deformations will be compared to some experimental results obtained with similar laser energy. The influence of hydrodynamic process and of equation of state will be shown on some parameter variations. 


\section{(Thursday, 19 August)}

\section{12: Structural dynamics in laser-excited materials studied with ultrafast $X$-ray scattering}

\section{(Invited)}

Presenter : Klaus. SOKOLOWSKI -TINTEN

Dr. Klaus Sokolowski-tinten (Universität Duisburg-Essen, Germany)

Upon intense excitation with ultrashort laser pulses materials are driven into highly non-equilibrium states and can undergo structural changes on very rapid time-scales. Due to the unique combination of atomic-scale spatial and temporal resolution, the recent progress in the development of ultrafast short wavelength sources has provided new opportunities for studying such processes. This talk will discuss some examples of our recent work using ultrafast time-resolved diffraction/scattering with laser-driven as well as accelerator-based femtosecond short wavelength (XUV- and X-ray) sources. This includes (i) studies of the laser-driven anti-ferromagnetic to ferromagnetic phase transition in FeRh, (ii) observation of the transient reversal of the Peierls-distortion in Bi upon strong optical excitation, and (iii) coherent XUV-scattering experiments during fs laser induced melting and ablation.

\section{27: Laser processing with short and ultrashort laser pulses}

Presenter : Peter BALLING

Mr. BALLING, Peter (Department of Physics and Astronomy, University of Aarhus), BYSKOV-NIELSEN, Jeppe (Department of Physics and Astronomy, University of Aarhus) ; CHRISTENSEN, Bjarke H (Department of Physics and Astronomy, University of Aarhus) ; CHRISTENSEN, Martin S. (Department of Physics and Astronomy, University of Aarhus) ; CHRISTENSEN, Mehrnaz N. (Department of Physics and Astronomy, University of Aarhus) ; FRISLEV, Martin (Department of Physics and Astronomy, University of Aarhus) ; SAVOLAINEN, Juha-matti (Department of Physics and Astronomy, University of Aarhus) ; SMITH, Christian (Department of Physics and Astronomy, University of Aarhus) ; WæDEGAARD, Kristian J. (Department of Physics and Astronomy, University of Aarhus)

Pulsed lasers are extremely useful tools for precision processing. The ongoing development of laser technology continues to expand the range of laser parameters that are available. This means that for a specific process targeted, one can choose a laser with a certain wavelength, pulse duration, repetition rate, and average power. In this presentation, it will be discussed how the physical mechanisms behind a given process in combination with the desired outcome can be used to devise the specifications for an appropriate laser system. For metals, the choices are strongly influenced by a relatively high thermal conductivity. This means that the laser pulse duration is coupled to the achievable resolution. In addition, the average power can also be an important parameter due to the physical consequences of an overall heating of the sample. Dielectrics can be processed with light of sufficiently high photon energy to bridge the band gap. They can, however, also be treated with lasers with a sufficiently short pulse duration, since the excitation can in this case proceed by non-linear absorption of the light. This may be useful for precision surface structuring and in addition opens the possibility of volume modifications. The talk will include several examples of laser processing from our group encompassing both 
fundamental investigations of light-matter interactions as well as applications within micro-mechanical interlocking, fiber-grating writing, and laser-written holography.

\section{28: Chemistry of monolayer functionalizations of silicon studied by laser-induced desorption mass spectrometry}

Presenter : Peter HESS

Prof. HESS, Peter (University of Heidelberg), Dr. SALINGUE, Nils (University of Heidelberg); Dr. LINGENFELSER, Dominic (University of Heidelberg) Presenter : Prof. HESS, Peter (University of Heidelberg)

Laser-induced thermal desorption (LITD) is an attractive method to investigate the stability and chemical bonding in monolayers of organic and inorganic functionalizations. The time-of-flight (TOF) analysis of well-defined H- and D-terminated $\mathrm{Si}(111)-\left(\begin{array}{ll}1 & 1\end{array}\right): \mathrm{H}(\mathrm{D})$ surfaces was used to calibrate the desorption temperature for different laser pulse energies of the XeCl-laser (309 $\mathrm{nm})$ employed. This procedure is based on the assumption that a monolayer has a negligible influence on laser heating of the substrate. Since the TOF temperature and time-of-flight are known, the masses of the emitted species formed during the desorption process can be determined, and thus provide a mechanistic understanding of the bond-breaking processes and fragmentation pattern of the chemisorbed groups.

A large variety of monolayers was studied ranging from atoms to complex organic end groups. As atomic terminations of the $\mathrm{Si}(111)-\left(\begin{array}{ll}1 & 1\end{array}\right)$ surface we investigated $\mathrm{H}, \mathrm{D}, \mathrm{Cl}, \mathrm{Br}$, and $\mathrm{I}$. Here the bonding stability and etching effects occurring during desorption were investigated. In addition, small organic end groups such as dimethylsiloxane and trimethylsiloxane but also larger hydrocarbons were studied. It is shown that well defined reactions can be initiated in organic monolayers such as a clean retro Diels-Alder cleavage, if a norbonen compound is irradiated at fluences of $110-200 \mathrm{~mJ} / \mathrm{cm} 2$. These reactions provide double bonds as reactive surface moieties, which can be used for controlled second

\section{13: New opportunities at XFEL (Invited)}

Presenter : Christian BRESSLER

Dr. BRESSLER, Christian (European XFEL GmbH, D-22607 Hamburg, Germany)Co-authors : GALLER, Andreas (European XFEL GmbH, D-22607 Hamburg, Germany) ; GAWELDA,Wojciech (European XFEL GmbH, D-22607 Hamburg, Germany) ; CHERGUI, Majed (EPF Lausanne, ISIC Bât. CH, CH-1015 Lausanne) ; MILNE, Chris (EPF Lausanne, ISIC Bât. CH,CH-1015 Lausanne) ; PHAM,, Van-thai (EPF Lausanne, ISIC Bât. CH, CH-1015 Lausanne) ; VANDER VEEN, Renske (EPF Lausanne, ISIC Bât. CH, CH-1015 Lausanne) ; JOHNSON, Steven (Paul-Scherrer Institut, CH-5232 Villigen-PSI, Switzerland); ABELA, Rafael (Paul-Scherrer Institut, CH-5232 Villigen-PSI, Switzerland)

Femtosecond X-Ray Science is an emerging field aiming to deliver a detailed understanding of the ultrafast elementary steps in complex processes involving changes in nuclear, electronic and spin states. Such processes are vital ingredients in chemistry and biology, but also in technological applications, including efficient charge transport in solar energy converters and ultrafast switchable molecular magnets. This talk will present results obtained on a prototype spin transition phenomenon in aqueous $\mathrm{Fe}(\mathrm{bpy}) 3$. Optical techniques explore the ultrafast changes in the valence states, but ultrafast $\mathrm{x}$ ray spectroscopies reveal the underlying nuclear and electronic changes during this spin transition 
process. While picosecond resolved XANES and EXAFS are exploited to understand the altered geometrical structure of the molecule after the spin transition is complete, Femtosecond XANES is able to monitor the evolution of this process in real-time. Finally, a recent experiment exploiting timeresolved XES of the $\mathrm{K}$ emission with picosecond resolution established a direct measurement of the short-lived (0.7 ns) high-spin state. Combing these spectroscopic tools with the intense intensity and femtosecond time resolution at x-ray free electron lasers will allow us to deliver a motion picture of the interplay between the nuclear, electronic and spin degrees of freedom during complex chemical reactions, and an outlook towards exploiting XFEL machines currently in operation will be given.

\section{14: Black and colored metals (Invited)}

Presenter : Chunlei GUO

Prof. GUO, Chunlei (University of Rochester)

Recently, we developed a number of techniques that allow us to dramatically alter properties of materials using high-intensity femtosecond lasers. The techniques led to the creation of the black and colored metals in my lab. In this talk, I will discuss our discoveries and various applications of the black and colored metals.

\section{29 : Dielectric to metal transitions in silicon and dielectrics observed during periodic surface structure formation induced by temporally tailored pulses}

Presenter : Ms. Magdalena FORSTER

Ms. FORSTER, Magdalena (University of Vienna, Department of Physical Chemistry, Währinger Str. 42, A-1090 Vienna, Austria), Mr. FAURE, Nicolas (Université Jean Monnet, Laboratoire Hubert Curien, F-42000 Saint Etienne, France) ; Prof. AUDOUARD, Eric (Université Jean Monnet, Laboratoire Hubert Curien, F-42000 Saint Etienne, France) ; Prof. STOIAN, Razvan (Université Jean Monnet, Laboratoire Hubert Curien, F-42000 Saint Etienne, France) ; Prof. KAUTEK, Wolfgang (University of Vienna, Department of Physical Chemistry, Währinger Str. 42, A-1090 Vienna, Austria)

Periodic surface structure formation on silicon, fused silica, sapphire and borosilicate glass was studied employing temporally tailored laser pulses, i.e. by variation of the pulse duration and the double pulse delay time. All these materials exhibited structures with periodicities close to the laser wavelength. They, except sapphire, showed a transition from parallel to perpendicular orientations in respects to electric field vector which is correlated with a transition from dielectric to metallic electronic behaviour controlled by fluence and pulse duration. Only sapphire and silicon yielded periodic surface structures with periodicities much smaller than the laser wavelength. The observations are correlated with structural and photoelectronic properties.

\section{30: Synthesis of high-pressure phases of oxides by laser-induced optical breakdown}

Presenter : Vygantas MIZEIKIS,

Dr. MIZEIKIS, Vygantas (Research Institute of Electronics, Shizuoka University, Japan), Dr. KOHARA, Shinji (Japan Synchrotron Radiation Research Institute, Japan) ; Dr. OHNISHI, Yasuo (Japan Synchrotron Radiation Research Institute, 
Japan) ; Dr. HIRAO, Norihisa (Japan Synchrotron Radiation Research Institute, Japan) ; Dr. SAITO, Akira (Division of Precision Science \& Technology and Applied Physics, Graduate School of Engineering, Osaka University, Japan); Dr. VAILIONIS, Arturas (Geballe Laboratory for Advanced Materials, Stanford University, USA) ; Prof. SAULIUS, Juodkazis (Centre for Micro-Photonics, Faculty of Engineering and Industrial Sciences Swinburne University of Technology,

Australia)

High-pressure and temperature phases of materials are highly sought in electronics, photonics, chemistry due to their unusual electrical, optical and mechanical properties. Here, we report on synthesis of compressed silica oxide phase using powerful shock-wave and thermal transientsgenerated via local optical breakdown induced by low-energy, tightly focused femtosecond laser pulses. Samples of silicon oxide (z-cut alpha-quartz) were patterned by femtosecond laser pulses with a 150 fs duration, a $800 \mathrm{~nm}$ central energy, and variable pulse energy of 140-170 $\mathrm{nJ}$ under focusing by a lens with numerical aperture $\mathrm{NA}=1.4$. Structural characterization of the patterned areas was performed by $\mathrm{X}$-ray diffraction (XRD) technique using a $50.323 \mathrm{keV}$ beam with a wavelength 0.233 angstrom of the BL04B2 beam line at SPring8 synchrotron facility. According to XRD analysis, laser-modified regions were found to contain a new high-pressure phase of silica which was theoretically predicted to form at the pressure of $120 \mathrm{GPa}$, and is expected to be one of several possible metastable high-pressure phases. This is the first experimental proof of the given phase. The optical breakdown technique can provide an accessible method for extreme high-pressure and temperature modification of other oxides and optically transparent solids in general.

\section{31: Energy coupling localization and selective laser processing in thin-film structures}

\section{Presenter : Gediminas RACIUKAITIS}

Dr. RACIUKAITIS, Gediminas (Center for Physical Science and Technology) Co-authors : GECYS, Paulius (Center for Physical Science and Technology) ; GEDVILAS, Mindaugas (Center for Physical Science and Technology)

Selectiveness of laser processing is top-most important for applications in thin-film electronics, including photovoltaics. Coupling of laser energy in multilayered thin-film structures depending on photo-physical properties of the layers and laser wavelength was investigated experimentally and theoretically. Selection of the right laser wavelength enabled keeping the energy coupling in a well defined volume at the interlayer interface. High absorption at inner interface of the layers triggered the localized temperature increase. Transient stress caused by the rapid temperature rise facilitated peeling of the films rather than evaporation.Simulation results are consistent with the experimental data achieved in laserscribing of copper-indium-gallium diselenide (CIGS) solar cells on flexible polymersubstrate using picosecond-pulsed lasers. Reaction of the films to laser irradiation was conditioned by optical properties of the layers at wavelength of laser radiation. In the CIGS solar cell structure with a thick ITO top-contact, the $355 \mathrm{~nm}$ laser radiation was mainly absorbed at the buffer layer, and the upper- most layer was removed by peeling rather than evaporation. The effect was even more evident when using $532 \mathrm{~nm}$ wavelength when laser radiation was coupled even deeper at the CIGSinterface. Ultra-short pulses ensured a high energy input rate into absorbing material permitting peeling of the layers without influence on the remaining material. 


\title{
(Friday, 20 August)
}

\section{15: Optical Near-Fields of Nanostructures: Characterization and Patterning (Invited)}

\author{
Presenter : Prof. Paul LEIDERER
}

Prof. LEIDERER, Paul (Universität Konstanz)

Illumination of small particles and nanostructures with laser light gives rise to interesting phenomena due to optical near fields, which can be exploited for various applications in nanotechnology. Knowledge about the distribution of the optical near fields of nanostructures is crucial when it comes to applications, but is also interesting from the fundamental point of view. We report here on three techniques for the imaging of optical near fields, in which the local field-induced change of the material in the vicinity of the nanostructure has been used: i) thin films of phase change media like GeSbTe, which can be switched between a crystalline and an amorphous phase; ii) the local ablation of a very smooth substrate like a silicon waver; iii) two-photon photo-polymerization, which, as a nonlinear process, preferentially takes place in locations where the optical near field is high. We show examples of the optical near field patterns both of dielectric objects (colloid spheres) and of metallic nano-triangles with different sizes. For the illumination of these objects individual pulses of a Ti:sapphire laser $(150 \mathrm{fs}, \lambda=800 \mathrm{~nm})$, but also other light sources have been used. The experimental results for the field distribution are compared to numerical simulations, and we find in general good agreement, but in some cases also pronounced discrepancies. In addition we demonstrate that one can make use of these optical near fields for efficient and simple nanostructuring.

\section{32 : Films of brookite titania nanorods deposited by matrix-assisted pulsed laser deposition for gas sensor applications}

Presenter : Anna Paola CARICATO

Dr. CARICATO, Anna Paola (Physics Department, University of Salento), Dr. BUONSANTI, Raffaella (2National Nanotechnology Laboratory (NNL) -Istituto di Nanoscienze del CNR) ; Dr. CATALANO, Massimo (Institute for Microelectronics and Microsystems, IMM-CNR) ; Dr. COZZOLI, Davide (National Nanotechnology Laboratory (NNL) Istituto di Nanoscienze del CNR) ; Prof. LUCHES, Armando (Physics Deparment, University of Salento) ; Dr. MANERA, Maria Grazia (Institute for Microelectronics and Microsystems, IMM-CNR) ; Prof. MARTINO, Maurizio (Physics Department, University of Salento) ; Dr. RELLA, Roberto (Institute for Microelectronics and Microsystems, IMM-CNR) ; Dr. TAURINO, Antonietta (Institute for Microelectronics and Microsystems, IMM-CNR)

In this paper we report on the laser deposition and characterization of titanium dioxide (TiO2) nanorod thin films to be used as sensitive elements in gas sensor devices. TiO2 nanorods in the brookite phase, with mean dimensions of $5 \mathrm{~nm} 50 \mathrm{~nm}$, covered with an oleate/oleyl amine capping layer, were synthesized by a recently developed wet-chemical route. The films were deposited by the MAPLE technique. A toluene nanorod solution $(0,016 \mathrm{wt} \% \mathrm{TiO} 2)$ was frozen at the liquid-nitrogen temperature and irradiated with a $\mathrm{KrF}$ excimer laser at the fluence of $350 \mathrm{~mJ} / \mathrm{cm} 2$ and repetition rate of $10 \mathrm{~Hz}$. \&lt;100\&gt; single-crystal Si wafers, silica slides, $\mathrm{Cu}$ carbon- coated grids and alumina interdigitated slabs were used as substrates to allow different characterization techniques to be applied. High-resolution scanning and transmission electron microscopy investigations evidenced the formation 
of quite rough films incorporating individually distinguishable $\mathrm{TiO} 2$ single nanorods and crystalline spheres with a mean diameter of $40 \mathrm{~nm}$. Films fabricated employing 6000 laser pulses had an average thickness of $120 \mathrm{~nm}$ and achieved a complete coverage of the substrate. Fourier-transform infrared spectroscopy confirmed the presence of both organic and inorganic components in the films, while on the basis of UV-Visible transmission and reflection spectra the optical properties of the films were evaluated. Very promising sensing responses to $1 \mathrm{ppm}$ of $\mathrm{NO} 2$ were obtained uponilluminating the samples with an UV lamp.

\section{33: Characterization of laser induced crystallization processes of (Ag)-Sb-S thin films towards optical data recording}

Presenter : Dr. Jan GUTWIRTH

Dr. GUTWIRTH, Jan (University of Pardubice) Co-authors : Prof. WáGNER, Tomáš (University of Pardubice) ; Dr. BEZDIčKA, Petr (Academy of Sciences of the Czech Republic v. v. i.) ; Prof. FRUMAR, Miloslav (University of Pardubice)

Rewritable optical data recording is one of the recent applications of thin chalcogenide films. Thin amorphous (Ag)-Sb-S films were prepared by thermal evaporation (TE) of Sb-S bulk sample and optically induced diffusion and dissolution (OIDD) of $\mathrm{Ag}$ into these thin $\mathrm{Sb}-\mathrm{S}$ films. This procedure allows to obtain sets of samples with particular Sb:S ratio and increasing content of Ag. Thin films' properties important for optical data storage were determined by UVVis-NIR Spectroscopy (optical properties, thickness) and Differential Scanning Calorimetry (thermal properties). Crystallization behavior of deposited thin films was tested via Ar+ ion laser irradiation. Deposited thin films as well as dot laser exposures were characterized by Energy Dispersive X-Ray analysis coupled with Scanning Electron Microscope (composition, chemical homogeneity, surface morphology) and by X Ray Diffraction (crystallinity). Dot laser exposures are moreover characterized by several optical microscopy techniques and by local measurement of optical transmission. Influence of composition and Ag content on optical and thermal properties and namely on laser induced crystallization processes are determined. Crystallization behavior of prepared thin films was described and discussed.

\section{16: Adaptive nano optics und spatiotemporal control (Invited )}

Presenter : Walter PFEIFFER

Prof. PFEIFFER, Walter (Universität Bielefeld)

The most general investigation and exploitation of light-induced processes require simultaneous control over spatial and temporal properties of the electro-magnetic field on a femtosecond time- and nanometer length-scale. Based on the combination of polarization pulse shaping and time-resolved two-photon photoemission electron microscopy we demonstrate such control over nanoscale spatial and ultrafast temporal degrees of freedom of an electromagnetic excitation in the vicinity of a nanostructure. The time-resolved cross-correlation measurement of the local photoemission yield reveals the switching of the nano-localized optical near-field distribution with a lateral resolution well below the diffraction limit and a temporal resolution on the femtosecond time scale [1]. In addition, 
successful adaptive spatiotemporal control demonstrates the flexibility of the method [2]. This flexible simultaneous control of temporal and spatial properties of nanophotonic excitations opens new possibilities to tailor and optimize the light-mater interaction in spectroscopic methods as well as in nanophotonic applications. The underlying control mechanisms are discussed and demonstrated.

[1] M. Aeschlimann et al., Proc. Natl. Acad. Sci. USA 107, 5329 (2010).

[2] M. Aeschlimann et al., Nature 446, 301 (2007)

\section{17: Accurate ellipsometric thickness measurement of alkyl monolayers in real time during VUV laser oxidation (Invited )}

Presenter : Peter HESS

Prof. HESS, Peter (University of Heidelberg), Dr. PRUNICI, Pavel (University of Heidelberg) Presenter : Prof. HESS, Peter (University of Heidelberg)

Ellipsometric thickness measurements are a highly sensitive optical tool to obtaininformation on selfassembled monolayers (SAMs) and directed assembly of organic monolayers on the nanoscale. Usually, single frequency ellipsometry is employed to study layers of long C18-chains. By using spectroscopic ellipsometry (IR-UV) and simultaneous laser processing thickness determinations can be extended into the sub nanometer range to study chemisorbed species containing only a few carbon atoms. The highest sensitivity and accuracy can be achieved by monitoring in situ and inreal time the thickness change occurring during VUV laser-induced oxidation of hydrocarbon moieties. With this technique a sensitivity of $\sim 0.02 \mathrm{~nm}$ and accuracy of $\sim 0.05 \mathrm{~nm}$ could be realized. Real-time detection was applied to investigate chemically bonded layers such as dimethylsiloxane (DMS) and trimethylsiloxane (TMS) groups, and longer alkyl chains $(\mathrm{CH} 3(\mathrm{CH} 2) \mathrm{n}-\mathrm{Si}(\mathrm{CH} 3) 2-)$ with $\mathrm{n}=17,11,9$, 7, 3 connected via siloxane bridges to oxidized silicon. Efficient oxidation of the organic end groups was achieved with pulsed F2-laser radiation $(157 \mathrm{~nm})$ in pure oxygen. While the length of octadecyltrichlorosilane and octadecyldimethylmonochlorosilane, employed as coupling agents, were quite similar the measured thicknesses of the functionalizations were $\sim 2.3 \mathrm{~nm}$ and $\sim 0.8 \mathrm{~nm}$, respectively, owing to self and directed assembly. For the small DMS and TMS terminations thicknesses of $0.24 \mathrm{~nm}$ and $0.20 \mathrm{~nm}$ were extracted.

\section{34: Tailoring the optical properties of $\mathrm{ZnO}$ thin films by doping and co-doping}

Presenter : . George EPURESCU

Dr. EPURESCU, George (National Institute for Lasers,Plasma and Radiation Physics) Co-authors : Dr. BIRJEGA, Ruxandra (National Institute for Lasers,Plasma and Radiation Physics) ; Dr. GALCA, Aurelian Catalin (National Institute for Materials Physics)

Thin films of $\mathrm{ZnO}$ doped with Magnesium and co-doped with Nitrogen and Magnesium were deposited by Pulsed Laser Deposition (PLD) and RF plasma beam assisted PLD in Oxygen or Oxygen-Nitrogen discharge for different Nitrogen/Oxygen ratios. A Nd:YAG laser working at $266 \mathrm{~nm}, 10 \mathrm{~Hz}$ was used for the depositions at an energy density of $3 \mathrm{~J} / \mathrm{cm} 2$ and $100 \mathrm{~W}$ RF power. X-ray Diffraction (XRD), Atomic Force Microscopy (AFM) and Spectroscopic Ellipsometry (SE) are employed to investigate the samples. The solubility of $\mathrm{Mg}$ in $\mathrm{ZnO}$ was increased up to $30 \%$ when assisting with $\mathrm{RF}$ plasma beam 
while the degree of cristalinity decreases with the increase of $\mathrm{Mg}$ concentration. The band-gap of the samples varies from 3.37 up to 3.57 when increasing the $\mathrm{Mg}$ concentration and the Nitrogen/Oxygen ratio for each $\mathrm{Mg}$ concentration. Segregation of $\mathrm{MgO}$ phase at a $\mathrm{Mg}$ concentration of $30 \%$ is detected both by XRD and SE for the films grown by PLD. A dependence of the dielectric function on both stoichiometry and degree of crystalinity is also present, the refractive index having values between 1.7 and 2 in visible spectral range.

NN

\section{36: Synthesis of gallium nitride nanocrystallites by pulsed laser ablation in background gas of pure nitrogen}

Presenter : Takehito YOSHIDA

Prof. YOSHIDA, Takehito (Dept. of Mechanical Engineering, Anan National College of Technology), Mr. KAKUMOTO, Soichiro (Department of Physics, Konan University) ; Prof. UMEZU, Ikurou (Department of Physics, Konan University) ; Prof. SUGIMURA, Akira (Department of Physics, Konan University)

$\mathrm{GaN}$ is widely significant material as not only light-emitting and high frequency devices but also photocatalysts. In this study, GaN nanocrystallites have successfully been synthesized in gas phase condensations excited by pulsed laser ablations (PLA), where safe and inactive pure N2 was used as a reactive background gas.

The third harmonics beam of Q-switched Nd:YAG laser (wavelength: $355 \mathrm{~nm}$, energy density: 4 $\mathrm{J} /(\mathrm{cm} 2$ pulse)) was focused onto a sintered high density GaN target. Pure N2 gases were introduced into the chamber and were maintained at constant pressures 0.05-20.0 Torr, using a differential evacuation system. SiO2 substrates were positioned normal to the target. The substrates and the chamber were not heated. It was clarified that the formed GaN nanocrystallites had hexagonal system, wurtzite structure, using a XRD method. The crystallinity depended on the background N2 gas pressures. The diffraction peak intensities increased with increasing N2 gas pressures.

We found that the PLA plasma (hyper thermal fields) can excited the inactive N2 gas into radicals and ions, even though the binding energy of the triple bond in N-N molecular is $9.8 \mathrm{eV}$. Furthermore, the activated nitrogen caused special confinement of the ablated species in high-pressure and hightemperature region. Facilitated nitridation in this region would suppress nitrogen deficiency in the condensed nanocrystallites. 


\title{
Abstracts of posters presentations
}

\author{
P 1: Long-range surface plasmon electro-optic polymer light modulator based on grating- \\ waveguide structure
}

Presenter : Prof. Wen-kai KUO

Prof. KUO, Wen-kai (National Formosa University)

Since the excitation of surface plasmon wave is under a resonance condition, it is highly sensitive to the refractive index change of the adjacent dielectric layer. Hence, the SPW method has been widely applied to biosensor by detecting the bio-layerRI change caused by biomolecular interaction. Besides, the SPW method also has greatpotential in optoelectronic devices. In this paper, a light modulator model basedcombination of electro-optic polymer and long-range surface plasmon resonant gratingwaveguide structure is numerically investigated. By using a grating structure, anincident light is coupled to excite surface plasmon on both side of a thin metallayer which is sandwiched between a cladding layer and the electro-optic polymer. Thickness effects of the grating structure and the cladding layer are investigated.An embodiment of the modulator is presented to be able to vary the reflectance from 0 to 0.6 for applying $2.1 \mathrm{~V}$ across a $1-\mu \mathrm{m}$ thick electro-optic polymer film with alinear electrooptic coefficient of $200 \mathrm{pm} / \mathrm{V}$.

\section{$P$ 2: Ordered arrays of nanopillars created by ultrashort laser nanoprocessing in the far and near field regime}

Presenter : Magdalena ULMEANU

Dr. ULMEANU, Magdalena (National Institute for Laser, Plasma and Radiation Physics, Bucharest, Romania), Mrs. FILIPESCU, Mihaela (National Institute for Laser, Plasma and Radiation Physics, Bucharest, Romania) ; Mr. RUSEN, Laurentiu (National Institute for Laser, Plasma and Radiation Physics, Bucharest, Romania) ; Dr. ZAMFIRESCU, Marian (National Institute for Laser, Plasma and Radiation Physics, Bucharest, Romania)

We report on the preparation of ordered arrays of spin valves based on current- induced magnetization switching (CIMS) nanopillars based on $\mathrm{Co} / \mathrm{Cu} / \mathrm{Co}$ trilayer by combination of ultra-high vacuum (UHV) deposition, laser nanoprocessing in the far and near field regime. The multilayer sequence will be deposited in an UHV (\&lt;10-7 Pa) electron beam deposition system and will be analyzed by different characterization techniques. A femtosecond laser with the wavelength $\lambda=775 \mathrm{~nm}$ and pulse duration $=200 \mathrm{fs}$ will be used for the nanoprocessing of the multilayer structure. The dependence of the morphology of the periodic structures on the experimentalconditions such as laser fluence and laser scanning speed will be investigated. To further decrease the size of the created periodical structures below $100 \mathrm{~nm}$ we will employ near field laser processing by using colloidal lithography technique. Such nanoprocessing method suggests a possible technique to produce ordered arrays of CIMS nano pillars. 


\title{
P 3: Study of polyethylene nanolayers by evanescent light microscopy
}

\author{
Presenter : Ion N MIHAILESCU
}

Prof. MIRCHIN, N. (HIT), Dr. POPESCU, S.a. (HIT) ; Dr. APTER, B. (HIT) ; Mr. GORODETSKY, U. (HIT) ; Dr. LAPSKER, I. (HIT) ; Prof. PELED, A. (HIT) ; Dr. DUTA, L. (National Institute for Lasers, Plasma and Radiation Physics, Lasers Department) ; Dr. DORCIOMAN, G. (National Institute for Lasers, Plasma and Radiation Physics, Lasers Department) ; Dr. POPESCU, A. (National Institute for Lasers, Plasma and Radiation Physics, Lasers Department) ; Prof. MIHAILESCU, I.n. (National Institute for Lasers, Plasma and Radiation Physics, Lasers Department)

We report in this work on the polyethylene thin films morphology investigation by Differential Evanescent Light Intensity (DELI) imaging method.The films were prepared by the Matrix Assisted Pulsed Laser Evaporation (MAPLE) technique. MAPLE was suitable for the synthesis of the organic materials in form of nanostructures. To prepare targets, $3 \%$ polyethylene powder was introduced in chloroform and frozen at liquid nitrogen temperature. We used a $\operatorname{KrF}$ excimer laser source $(\lambda=248$ $\mathrm{nm}, \tau=25 \mathrm{~ns}$ ) and the incident laser intensity was set at $0.4 \mathrm{~J} / \mathrm{cm} 2$. In MAPLE the organic material is protected by the frozen solvent against the direct laser and plasma action causing irreversible damage. Insulating polyethylene thin films of different thicknesses, below $200 \mathrm{~nm}$, were fabricated by varying the deposition conditions. We applied the DELI microscopy technique as a fast and low cost method for morphology investigation of relatively large polyethylene nanolayer areas. A phenomenological model for the interaction between the evanescent waves and the deposited material is presented as well as simulation of the nanofilms imaging inferred by Maxwell equation solver Full WAVE .

\section{P 4: z-Scan Characterization of zwitterionic chomophores for optoelectronic switching}

Presenter : Ulrich SKRZYPCZAK

Mr. SKRZYPCZAK, Ulrich (Martin Luther University of Halle-Wittenberg, Centre for Innovation Competence SiLinano ${ }^{\circledR}$, Karl-Freiherr-von-Fritsch-Str. 3, 06120 Halle (Saale), Germany) Dr. WILLIAMS, Grant. V. M. (Industrial Research Ltd., P.O. Box 31310, Lower Hutt 5040, New Zealand) ; Dr. MICLEA, Manuela (Martin Luther University of HalleWittenberg, Centre for Innovation Competence SiLi-nano®, Karl-Freiherr-von-Fritsch-Str. 3, 06120 Halle (Saale), Germany) ; Mr. BHUIYAN, Delower (Industrial Research Ltd., P.O. Box 31310, Lower Hutt 5040, New Zealand) ; Mr. JANSEENS, Stefaan (Industrial Research Ltd., P.O. Box 31310, Lower Hutt 5040, New Zealand) ; Dr. SCHWEIZER, Stefan (Martin Luther University of Halle-Wittenberg, Centre for Innovation Competence SiLi-nano ${ }^{\circledR}$, Karl-Freiherr-vonFritsch-Str. 3, 06120 Halle (Saale), Germany and Fraunhofer Center for Silicon Photovoltaics, Walter-Hülse-Str. 1, 06120 Halle (Saale), Germany)

Materials with high 2nd- and 3rd-order nonlinear optical (NLO) susceptibilities are of great interest for applications in telecommunications as optical switches, reconfigurable add/drop multiplexers, and wavelength switching devices. Besides the high NLO susceptibility, other factors such as transparency at the operating wavelengths as well as thermal stability and photostability are important for such applications. Conventional solid-state compounds are frequently limited by their high power requirements caused by their relatively weak NLO response. In contrast, organic chromophores provide a NLO response, which is several orders of magnitude larger than that found in commonly used materials.A series of NLO chromophores have been developed at Industrial Research Ltd. Anexample is the chromophore PYR-3 that has been mixed with amorphous polycarbonate toproduce thin and thick films. Hyper-Rayleigh scattering measurements and the calculated dipole moments of PYR-3 
yielded a molecular NLO figure of merit that is considerably higher than that of lithium niobate.To sustain these promising results with respect to the NLO response of the namedchromophores, nonlinear z-scan experiments have been carried out using a highintensity femtosecond laser. From these measurements the nonlinear refractive index as well as an estimate of the two- and three-photon absorption coefficients was obtained. The results emphasize the remarkable macroscopic 3rd-order nonlinear response of PYR-3.

\section{P 5: Photon-assisted surface photovoltage in thermally oxidized metal-contaminated n-type silicon wafers}

Presenter : Hirofumi SHIMIZU

Prof. SHIMIZU, Hirofumi (College of Engineering, Nihon University) Co-authors : Mr. NAGASE, Shintarou (College of Engineering, Nihon University) ; Dr. IKEDA, Masanori (College of Engineering, Nihon University)

A photon-assisted surface photovoltage (SPV) appears when a depletion or inversion layer is formed on a Si semiconductor surface by irradiating it with a photon beam (PB). The depletion or inversion layer can be created either by an atomic bridging formation in the $\mathrm{SiO} 2$ or by a Schottkybarrier on the bare Si surface. In this work, we used the frequency-dependent alternating current (AC) SPV method to investigate the transition from the Schottky-barrier type- to the atomic bridging typeinduced SPV in SiO2 grown on a Cr-contaminated Si surface. On irradiation with the PB (wavelength: $470 \mathrm{~nm}$ ), a Schottky-barrier type AC SPV was produced at room temperature. In this case, the hydrophobic Si surface had been contaminated with a $\mathrm{Cr}$ aqueous solution $(10 \mu \mathrm{g} / \mathrm{mL})$, resulting in a $\mathrm{Cr}$ concentration on the Si surface of $1.5 \times 1015$ atoms $/ \mathrm{cm} 2$. In the early stages of thermal oxidation at $373 \mathrm{~K}$, the AC SPV vanished, indicating that the Schottky-barrier had collapsed. In contrast, a frequency-dependent AC SPV clearly appeared as the oxidation was continued (longerhan $60 \mathrm{~min}$ ), indicating that the atomic bridging mechanism had begun to work in the form of the negative network (CrOSi)- in the SiO2. At temperatures higher than $473 \mathrm{~K}$, the AC SPV was found to be inversely proportional to frequency (f) which corresponds to weak or strong inversion. It can be concluded that for Cr-contaminated Si surfaces, the occurrence of the SPV is dependent on temperature for either mechanism.

\section{P 6: Extended two-temperature model for covalent materials}

Presenter: Carolina TRICHET PAREDES

TRICHET PAREDES, Carolina (Stuttgart University) Co-authors : SONNTAG, Steffen (Stuttgart University) ; ROTH, Johannes (Stuttgart University); TREBIN, Hans-rainer (Stuttgart University)

We present an extension of the standard two-temperature model (TTM) for metals to the case of covalent materials. Our analysis is based on the model introduced by Lietoila and Korfiatis (J.Phys.D. 40 (2007) 6803) in their studies of silicon. The simple yet accurate picture considered there is able to explain the interaction between the lattice and the plasma formed due to the radiation absorbed in the sample. The change of carrier density and carrier energy is related to the absorption coefficient and recombination factor. The connection to the ordinary two-temperature model is through the definition 
of the carrier energy: $\$ E_{-}\{$carr $\}=3 n k \_B T \_\{c a r r\} \$$. Considering only the electronic charge carrier temperature \$T_e\$, we can calculate \$T_e\$ via the two-temperature model and transfer it to molecular dynamics via the electron-phonon-coupling. With this definition we have a realistic picture of the sample we want to study. The next step will be to compare the simulation results obtained with this model to experimental observations.

\section{P 7: Disorder and leakage influence on slow light phenomena in coupled resonator and photonic crystal waveguides}

Presenter: Søren RAZA

GRGIC, Jure (DTU) Co-authors : Mr. GOOR PEDERSEN, Jesper (DTU) ; XIAO, Sanshui (DTU) ; RAZA, Søren (DTU) ; CAMPAIOLI, Enrico (DTU) ; MORTENSEN, Asger Niels

In the past decade slow light (SL) has attracted big attention. Potentially, almost all optical devices could take advantage of SL phenomena in order to improve their performances or allow miniaturization. Unfortunately, there is always some presence of disorder in real samples, e.g. due to imperfections from fabrication processes. Materials that are used in fabrication will also lead to dissipation of energy in the form of absorption or radiation. While dispersion and loss are often independent issues, the SL regime is different and may influence loss and vice versa. In order to design robust structures it is thus necessary to understand the more fundamental interplay between imperfections and SL. We employ several simplified models to point out the fundamental limitations on SL propagation and group-delay dynamics, with the important conclusion that group index and imperfections has to be treated on an equal footing in order to be able to take full advantage of SL designs in future applications.

\section{P 8: Photo-assisted production of refined beams of neutral free radicals for selective epitaxy of metastable condensed phases}

Presenter : Keiji HAYASHI

Prof. HAYASHI, Keiji (Graduate School of Engineering, Kanazawa Institute of Technology), Dr. TANAKA, Daisuke (Graduate School of Engineering, Kanazawa Institute of Technology) ; Dr. TOYODA, Kazuho (Graduate School of Engineering, Kanazawa Institute of Technology)

In order to further evolve quantum-functional nano-devices using metastable condensed phases, it has become indispensable in recent years to ingeniously utilize selective surface reactions of labile chemical species such as neutral free radicals for the device processing. The problem encountered in the precise experimental study of a chemical reaction between a neutral free-radical species and a well-characterized material surface is how to supply a sufficient-flux continuous beam of the desired neutral free-radical species onto the surface.

We have been developing several experimental production approaches, two of which are photo-deionization of negative ion beams ( PDINIB ) and photo-dissociation of energetic compound beams ( PDECB ) [1]. In an application of the PDINIB method to the doping of $\mathrm{Cr}$ atoms during the low-temperature epitaxial growth of $(\mathrm{Al}, \mathrm{Ga}) \mathrm{N}$ by the PDECB-based approach, we found enhancement 
of incorporation of the $\mathrm{Cr}$ atoms into the growing surface of the diluted magnetic semiconductor (Al,Ga,Cr)N.

At the ICPEPA-7, we will discuss fundamental aspects of the production of the refined beams of neutral free radicals ( RBNR ) by the PDECB and PDINIB methods.

This work is financially supported by the Ministry of Education, Culture, Sports, Science and Technology, Japan.

[1] Keiji Hayashi et al., Appl. Surf. Sci. 255 (2009) 9581.

\section{P 9: Effects of irradiation conditions on the lateral grain growth during laser crystallization of a- Si film on borosilicate glass substrates}

Sungho JEONG

Prof. JEONG, Sungho (Gwangju Institute of Science and Technology (GIST)) ; Mr. KIM, Pilkyu (Gwangju Institute of Science and Technology (GIST)), Prof. MOON, Seung-jae (Hanyang University)

Laser crystallization of amorphous silicon (a-Si) film is adopted to improve the performance of thin film transistor (TFT) devices and thus has been extensively investigated over the past years. Although glass is a highly cost effective material as a substrate, its actual use on laser crystallization has been limited by several reasons. For example, the a-Si layer on glass substrate is easily damaged if the thickness of a-Si layer becomes thinner than a few hundreds of micrometer, whereas a higher performance of the TFT device is achievable with thin a-Si films (\&lt; $100 \mathrm{~nm}$ ). Also, the impurity diffusion from the glass substrate to a-Si film can prevent the grains from growing to large sizes. In this work, we report the results for lateral grain growth of a-Si film on borosilicate (Pyrex) glass substrate using a dual laser irradiation method (CW preheating laser: wavelength $=532 \mathrm{~nm}$, pulse laser: wavelength $=532 \mathrm{~nm}$, pulse width $=5 \mathrm{~ns}$ ). We examined how the preheating condition and pulse laser energy affect on the crystallinity and grain size. Raman spectroscopy and scanning electron microscopy were used for the measurement of crystallinity and grain size, respectively. The specific resistance of grains produced at different irradiation conditions were estimated from the measured I-V curves and compared with the crystallinity. It is demonstrated that the lateral grain growth of a-Si films up to several tens of micrometer size is possible with glass substrate if the irradiation condition

\section{P 10: Growth and thermoelectric properties of FeSb2 films produced by pulsed laser deposition}

Presenter : Stela CANULESCU

Dr. SUN, Ye (Department of Chemistry and iNANO, University of Aarhus), Dr. CANULESCU, Stela (Department of Photonics Engineering, Risø Campus, Technical University of Denmark) ; Prof. SCHOU, Jørgen (Department of Photonics Engineering, Risø Campus, Technical University of Denmark) ; Prof. IVERSEN, Bo Brummerstedt (Department of Chemistry and iNANO, University of Aarhus)

$\mathrm{FeSb}_{2}$, a strongly correlated semiconductor, has promising application potential for thermoelectric cooling at cryogenic temperatures [1,2]. Single crystals of $\mathrm{FeSb}_{2}$ were found to exhibit colossal thermopower (S) values up to $\sim-45000 \mu \mathrm{VK}-1$ and record high power factors up to $2300 \mu \mathrm{WK}-2$ $\mathrm{cm}^{-1}$ at $12 \mathrm{~K}$ [2]. However, the thermoelectric performance of $\mathrm{FeSb}_{2}$ is restricted by its large lattice thermal conductivity $(\kappa \mathrm{L})$. Thin film thermoelectric materials could have a much reduced $\kappa \mathrm{L}$ 
due to surface and grain-boundary scattering of phonons. Therefore, $\mathrm{FeSb}_{2}$ thin films are expected to have remarkably enhanced thermoelectric performance. Herein, FeSb ${ }_{2}$ films were produced on silica substrates in a low-pressure Ar environment by a pulsed Nd:YAG laser at $355 \mathrm{~nm}$. The effect of growth parameters, such as substrate temperature, Ar pressure, incident fluence and growth time, on the PLD growth of $\mathrm{FeSb}_{2}$ was systematically studied. Uniform, continuous and nearly phase-pure $\mathrm{FeSb}_{2}$ films with thickness of 100-400 $\mathrm{nm}$ were produced. Thermal transport and Hall measurements were performed to study their thermoelectric transport properties. A maximum absolute value of $\mathrm{S} \sim 120$ $\mu \mathrm{VK}-1$ at $40 \mathrm{~K}$ was obtained. This study should serve to strengthen the interest in application of $\mathrm{FeSb}_{2}$ films in thermoelectrics.

$1 \quad$ P. Sun, N. Oeschler, S. Johnsen, B. B. Iversen, F. Steglich. Dalton Trans. 39 (2010) 965.

2 A. Bentien, S. Johnsen, G. K. H. Madsen, B. B. Iversen, F. Steglich, Europhys. Lett. 80 (2007) 17008.

\section{$P$ 11: Versatile tailoring of refractive index microstructures in iron-doped lithium niobate by direct laser writing technique}

Presenter : MIZEIKIS, Vygantas

: Dr. MIZEIKIS, Vygantas (Research Institute of Electronics, Shizuoka University, Mr. PURLYS, Vytautas (Laser research Center, Department of Quantum Electronics, Vilnius University, Lithuania) ; Prof. JUODKAZIS, Saulius (Centre for Micro-Photonics, Faculty of Engineering and Industrial Sciences, Swinburne University of Technology, Australia)

We report on application of direct laser writing (DLW) technique using femtosecond laser pulses in iron-doped lithium niobate, and demonstrate versatility of all-optical reversible tailoring of refractive index modulation structures. In iron-doped lithium niobate, optical excitation by spatially varying radiation can almost instantaneously create space-charge electric field between iron dopants and electrons photoexcited from dopant levels, and consequently, internal electric field arises leading to refractive index modulation via electro-optical effect. Single features and extended structures such as diffraction gratings were recorded by translating beam of a femtosecond Ti:Sapphire laser (pulse wavelength $800 \mathrm{~nm}$, temporal length \&lt;100 fs, repetition rate $80 \mathrm{MHz}$, average power \&lt; $70 \mathrm{~mW}$ ) focused by a lens with numerical aperture $\mathrm{NA}=0.35$. The induced refractive index modulation develops almost instantaneously, i.e., no prolonged exposure is required, as is the case with holographic recording, the index modulation is estimated to be about 0.01 , and the recorded features can be erased and overwritten many times. High amplitude of the index modulation, and reversibility of the recording process may open ways for all-optical formation of photonic micro-structures with nearly arbitrary topologies in the bulk of photorefractive crystals.

\section{$P$ 12: Studies of shrinkage as a result of holographic recording in acrylamide based photopolymer film}

Presenter : Mr. Mohesh MOOTHANCHERY

Mr. MOOTHANCHERY, Mohesh (Centre for Industrial \& Engineering Optics, Dublin Institute of Technology, Ireland) Dr. NAYDENOVA, Izabela (Centre for Industrial \& Engineering Optics, Dublin Institute of Technology, Ireland) ; Prof. TOAL, Vincent (Centre for Industrial \& Engineering Optics, Dublin Institute of Technology, Ireland) 
We studied the Bragg detuning of transmission diffractive gratings recorded at different slant angles in acrylamide based photopolymer at different intensities. Transmission diffraction gratings of spatial frequency 1000 lines/mm were successfully recorded in an acrylamide based photopolymer film having $60 \mu \mathrm{m}$ thickness. Kogelniks coupled wave theory was successfully used to fit the angular selectivity curves of the gratings. We have obtained the grating thickness and the final slant angles from the Bragg curve and hence calculated the shrinkage caused by holographic recording from the plot, tangent of final slant angle versus tangent of initial slant angle. The shrinkage of the material was evaluated for three different recording intensities 1,5 \&amp; $10 \mathrm{~mW} / \mathrm{cm} 2$, while the total exposure energy was kept constant- $80 \mathrm{~mJ} / \mathrm{cm} 2$. The shrinkage of the material is higher for recording with lower intensities and the corresponding values are $2.19 \%, 1.5 \%$ and $1.08 \%$. The next step is the modification of the photopolymer material in order to decrease the shrinkage due to photopolymerisation. We are studying the effect of incorporating different nanoparticles in the material.

\section{P 13: UV-VIS laser cleaning investigations of model contaminations on cellulose paper}

Presenter : Wolfgang KAUTEK

Prof. KAUTEK, Wolfgang (University of Vienna, Department of Physical Chemistry), Ms. FORSTER, M. (University of Vienna, Department of Physical Chemistry) ; Ms. ARIF, Sairah (University of Vienna, Department of Physical Chemistry) ; Mr. BUSHUK, S. (National Academy of Sciences of the Republic of Belarus, Institute of Physics) ; Mr. KOUZMOUK, A. (National Academy of Sciences of the Republic of Belarus, Institute of Physics) ; Mr. TATUR, H. (National Academy of Sciences of the Republic of Belarus, Institute of Physics) ; Dr. BATISHCHE, S. (National Academy of Sciences of the Republic of Belarus, Institute of Physics)

Contactless cleaning by lasers has become an important application field of laser materials processing in cultural heritage. Laser Cleaning basically is a phase separation process driven by laser radiation. The preservation of the paper substrates by the minimization of the penetration depth of light in relation to a limited heat-affected zone is discussed. Potentials of this new technique are correlated with approaches with visible wavelength treatments at a model contamination consisting of charcoal powder on pure cellulose paper. The employment of far-UV laser radiation at $213 \mathrm{~nm}$ led to a lowering of the destruction threshold of paper due to the photochemical action of high energy photons in contrast to visible photons $(532 \mathrm{~nm})$. Colorimetric lightness measurements served to quantify the cleaning status and the irreversible (photo) chemical modifications by measurement of yellowing. Yellowing and therefore chemical alterations have been observed at all optimum cleaning conditions at $532 \mathrm{~nm}$ in contrast to $213 \mathrm{~nm}$. A cleaning mechanism has been detected for $532 \mathrm{~nm}$ radiation in addition to the well-known evaporation of contaminants, which is lateral mechanical blasting.

\section{P 14: Combined 213+1440 nm laser ablation treatment of cornea}

Presenter : Wolfgang KAUTEK

Prof. KAUTEK, Wolfgang (University of Vienna, Department of Physical Chemistry), Dr. BATISHCHE, S. (National Academy of Sciences of the Republic of Belarus, Institute of Physics) ; Mr. BUSHUK, S. (National Academy of Sciences of the Republic of Belarus, Institute of Physics); Mr. KOUZMOUK, A. (National Academy of Sciences of the Republic of Belarus, Institute of Physics) ; Mr. SAVITCH, A. (National Academy of Sciences of the Republic of Belarus, Institute of Physics) ; Mr. TATUR, H. (National Academy of Sciences of the Republic of Belarus, Institute of Physics) ; Prof.

GRABNER, G. (Paracelsus Medical University (PMU), University Eye Clinic) 
UV laser cornea treatment technology for eye vision correction is of vivid interest. This study is focused on the processing improvement by a combined UV+IR laser cornea treatment. Thus, "dry" ablation of the cornea in naturally wet environment is achieved by applying an infrared $1440 \mathrm{~nm}$ beam of $70 \mu$ s pulse duration which is absorbed by the strong water absorption band around $1455 \mathrm{~nm}$. In the second step a thin quasi dry layer of ca. 1-3 $\mu \mathrm{m}$ thickness of corneal tissue is ablated by an ultraviolet $213 \mathrm{~nm}$ pulse of 10-15 ns duration. Freshly enucleated calf's eyes cut at constant depths to provide flaps with diameters of 6-8 $\mathrm{mm}$ were used. The $213 \mathrm{~nm}$ radiation was variably delayed relatively to the $1440 \mathrm{~nm}$ pulses. The pit depth, inlet area and its volume were experimentally determined and the laser volume and depth ablation rate were evaluated. Statistics shows that for all eyes the average pit depth and volume for the combined $213 \mathrm{~nm}+1440 \mathrm{~nm}$ treatment is up to $25 \%$ higher paralleled by an improved reproducibility as compared with the sole $213 \mathrm{~nm}$ treatment. These results show that combined IR-UV laser treatment possesses a substantial medical application potential.

\section{P 15: Nanoscale femtosecond laser processing of diamond}

Presenter : Magdalena FORSTER

Ms. FORSTER, Magdalena (University of Vienna, Department of Physical Chemistry, Waehringer Strasse 42, A-1090 Wien, Austria), Mr. HUBER, Christoph (University of Vienna, Department of Physical Chemistry, Waehringer Strasse 42, A-1090 Wien, Austria) ; Mr. TRETTENHAHN, Günter (University of Vienna, Department of Physical Chemistry, Waehringer Strasse 42, A-1090 Wien, Austria) ; Prof. KALISH, Rafi (Technion Institute of Technology, Solid State Institute and Physics Department, IL-32000 Haifa, Israel) ; Prof. KAUTEK, Wolfgang (University of Vienna, Department of Physical Chemistry, Waehringer Strasse 42, A-1090 Wien, Austria)

Nanocavities and self-assembled periodic nanostructures with dimensions below the diffraction limit on nitrogen-doped diamond were achieved by multiphoton excitation. High-numerical aperture focusing $(\mathrm{NA}=0.7)$ of 70 -fs-laser pulses $(800 \mathrm{~nm}, 1.55 \mathrm{eV}, 100 \mathrm{~nJ})$ was applied. Top-down structuring led to modifications of less than $500 \mathrm{~nm}$. We show that the low nanometer-scale diameter is caused by the interaction narrowing close to the Gaussian profile maximum and additionally the two photon absorption (bandgap=1,7eV). Bottom-up structuring with $11 \mathrm{MHz}$ fs-pulse trains could generate selfassembled regular structures with periodicities below $50 \mathrm{~nm}$ and around $200 \mathrm{~nm}$. In the $\mathrm{kHz}$ range with 10 - 100 pulses, however, only the ripple type with $200 \mathrm{~nm}$ could be observed. Due to the spot size below the diffraction limit the formation of two of these self-assembled features could be produced. This novel finding is in contrast to earlier results of ripple periodicities at $800 \mathrm{~nm}$ of down to ca. 400 $\mathrm{nm}$. Our $50 \mathrm{~nm}$ and $200 \mathrm{~nm}$ ripples generated with $800 \mathrm{~nm}$ are not far away from 50-100 and $250 \mathrm{~nm}$ periodicities generated with $248 \mathrm{~nm}$ and a pulse length of $380 \mathrm{fs}$ by other authors. Various models such as fs-laser-induced surface plasmon grid interaction with the laser field, non-thermal melting processes etc. will be discussed.

\section{P 16: Highly enhanced surface plasmon resonance in a coupled silver nanodumbbell}

Presenter : Chau YUAN-FONG

Prof. YUAN-FONG, Chau (Ching Yun University), MIN WEI, Chen (National Taiwan University) ; HAN-HSUAN, Yeh (Chin Yun University) ; DIN PING, Tsai (National Taiwan University)

Scattering field interactions and surface plasmon resonance in a coupled silver nanodumbbell (a pair of 
silver nanosphere connected by a silver nanobar) are simulated by using the finite-element method. The scattering cross section enhancement which exhibits a blue-shifted is associated with the diameter of the silver nanobar and the wavelength of incident light. Interestingly, the generated optical cloud exceeds two times of the nanodumbbell size which can be turned by varying the diameter of the silver nanobar. We investigate scattering field interactions and surface plasmon resonance in a coupled silver nanodumbbell (a pair of silver nanosphere connected by a silver nanobar) by using the finite-element method. The scattering cross section enhancement which exhibits a blue-shifted is associated with the diameter of the silver nanobar and the wavelength of incident light. Interestingly, the generated optical cloud exceeds two times of the nanodumbbell size which can be turned by varying the diameter of the silver nanobar.

\section{P 17: Investigation of fs-laser-induced phase transformations and atomic mixing in au film - cu substrate system}

Presenter : Roland HERGENROEDER

Dr. HERGENROEDER, Roland (Leibniz-Institut für Analytische Wissenschaften-ISAS), Dr. ZHIGILEI, Leonid (University of Virginia) ; Dr. GUREVICH, Evgeny (Leibniz-Institut für Analytische Wissenschaften-ISAS) ; WU, Chengping (University of Virginia) ; THOMAS, Derek A. (University of Virginia)

Laser ablation and processing is known to change material properties, e.g. material hardening or alloying of heterogeneous samples can be achieved in this way. This has been experimentally demonstrated for $\mathrm{CW}$ and nanosecond pulsed lasers long ago. Application of femtosecond lasers is a promising extension of this technique since this may be a key for producing alloys of immiscible compounds due to very rapid melting and solidification processes. Femtosecond laser pulses are absorbed in a thin layer and trigger quick sample heating with heating rate of approximately $1014 \mathrm{~K} / \mathrm{s}$ [1]. A shallow layer of metal melt appears and mixing of the metals constituting the sample starts. After that a very rapid (with cooling rates of more than $1012 \mathrm{~K} / \mathrm{s}$ ) resolidification occurs under such conditions. Such a rapid quenching allows supposing that even immiscible solids will not separate and exotic alloys may be produced. We present experimental and numerical study of surface metal mixing of $\mathrm{Au} / \mathrm{Cu}$ bimetal layers exposed to fs-laser pulses. Depth profiles are studied by means of X-ray photoelectron spectroscopy (XPS). We demonstrate formation of an internal, alloyed interface layer in a certain window of laser energies. As a second control parameter the number of shots per area is indentified controlling the depth of the mixing process. The results are compared with new temporal extended numerical molecular dynamics simulations.

[1] D.S. Ivanov and L.V. Zhigilei, Phys. Rev. B, 68, 064114(2003)

\section{$P$ 18: The structural modifications induced in lithium niobate and KDP crystals with high repetition rate femtosecond laser pulses}

Presenter : Domas PAIPULAS

Mr. PAIPULAS, Domas (Vilnius University, Laser Research Center, Lithuania), Dr. KUDRIAšOV, Viačeslav (Vilnius University, Laser Research Center, Lithuania) ; Mr. MALINAUSKAS, Mangirdas (Vilnius University, Laser Research Center, Lithuania) ; Prof. SMILGEVIčIUS, Valerijus (Vilnius University, Laser Research Center, Lithuania) ; Prof. 
SIRUTKAITIS, Valdas (Vilnius University, Laser Research Center, Lithuania)

The possibility to locally modify optical properties of various transparent materials by femtosecond laser pulses has been intensively studied in the recent decade. Numerous types of glasses where investigated and various modification regimes have been found. However, only recently it was demonstrated that similar modifications arealso possible in crystalline materials. We have studied the modifications induced in lithium niobate and KDP crystals withYb:KGW femtosecond laser pulses, having pulse duration of $300 \mathrm{fs}$ and repetition rateup to $300 \mathrm{kHz}$. By focusing laser beam with 0.42 numerical aperture objective we have recorded gratings having period of $5 \mu \mathrm{m}$ inside the bulk of the crystal by direct laser writing technique. The homogeneous gratings were formed in lithium niobate with pulse energies up to $1.5 \mu \mathrm{J}$, however the threshold for damage appearance strongly depends on stress in crystalline structure and cracks can appear on much lower energy values depending on crystal position. Afterwards, the sample was kept for 1 hour in $130 \mathrm{C}$ temperature, but no significant decrease of the written structures was observed, only these with writing energies less then $0.7 \mu \mathrm{J}$ disappeared. For KDP crystal the damage-free gratings were formed with energies around $0.18 \mu \mathrm{J}$, however after the same thermal treatment these structures completely disappeared. The results show that lithium niobate is very promising crystal candidate for microphotonics applications.

\section{P 19: Nanosecond laser ablation and deposition of silicon films}

Presenter :. Seong Shan YAP

Dr. YAP, Seong Shan (Norwegian Univeristy of Science and Technology) Mr. SIEW, Wee Ong (Multimedia University); Dr. LADAM, Cécile (SINTEF Materials and Chemistry) ; Dr. DAHL, Øystein (SINTEF Materials and Chemistry); Dr. WORREN REENAAS, Turid (Norwegian University of Science and Technology) ; Prof. TOU, Teck Yong (Multimedia University)

Nanosecond pulsed KrF (248 nm, $25 \mathrm{ns)} \mathrm{and} \mathrm{Nd:YAG} \mathrm{(1064} \mathrm{nm,} 532 \mathrm{~nm}, 355 \mathrm{~nm}, 5 \mathrm{~ns})$ lasers were used to ablate polycrystalline Si target in background pressure of \&lt;10-6 Torr. Si films were deposited on $\mathrm{Si}$ and GaAs substrates at room temperature. The surface morphology of the films was characterized by using scanning electron microscopy (SEM) and atomic force microscopy (AFM). Round droplets from $10 \mathrm{~nm}$ to $5 \mathrm{um}$ were detected on the deposited films. The micron sized droplets were crystalline and the films were amorphous as analyzed by Raman Spectroscopy. The dependence of the films properties on laser wavelengths and fluence is discussed.

\section{P 20: Maple prepared organic heterostructures for photovoltaic applications}

Presenter : Florin STANCULESCU

Dr. STANCULESCU, Anca (National Institute of Materials Physics, Romania), Ms. SOCOL, Marcela (National Institute of Materials Physics, Romania); Dr. STANCULESCU, Florin (University of Bucharest, Faculty of Physics, Romania) ; Prof. GIRTAN, Mihaela (Laboratoire de Photonique d'Angers, Université d'Angers, France) ; Dr. SOCOL, Gabriel (National Institute for Laser, Plasma and Radiation Physics, Romania) ; Prof. MIHAILESCU, Ion (National Institute for Laser, Plasma and Radiation Physics, Romania)

Matrix Assisted Pulsed Laser Evaporation (MAPLE) is a Pulsed laser evaporation technique, which uses as target a frozen solution of the molecules to be deposited in a highly volatile and light absorbing solvent. Thin films of low molecular weight organic semiconductors like ZnPc, PTCDA, Alq3 have been deposited on different substrates, single crystal silicon, ITO and quartz using as solvent 
(DMSO, chloroform) and characterized by spectroscopic (UV-VIS, FTIR, Photoluminescence) and microscopic (AFM, SEM) methods. To preserve the chemical structure of the compounds during the deposition, the effect of the deposition conditions (fluence, substrate temperature, number of pulses) on the properties of the films has been analysed. This paper also presents some investigations on the charge injection at the inorganic/organic interfaces and the electrical transport phenomena in sandwich type inorganic (ITO, Si)/PTCDA/Alq3/metal (Au, Al), emphasising the correlation between the surface morphology and the electrical properties. Double layer solar cell structures ITO/ZnPc/PTCDA(Alq3)/metal have been prepared using MAPLE technique for the deposition of organic layers and sputtering/vacuum evaporation for the deposition of the metallic contact. We have drawn the I-V characteristics in dark and under constant illumination at direct and reverse bias and the effect of the space charge or trapped charge phenomena on the electrical conduction in these heterostructures have been evidenced.

\section{$P$ 21: The microstructuring of optical fiber tip using the femtosecond laser for the multidirectional firing}

Presenter :. Ik-bu SOHN

Dr. SOHN, Ik-bu (Advanced Photonics Research Institute(APRI), Gwangju Institute of Science and Technology (GIST)) Dr. KIM, Youngseop (Advanced Photonics Research Institute(APRI), Gwangju Institute of Science and Technology (GIST)) ; Dr. NOH, Young-chul (Advanced Photonics Research Institute(APRI), Gwangju Institute of Science and Technology (GIST)) ; Prof. LEE, Ho (School of Mechanical Engineering, KyuongPook National University) ; Dr. KIM, Jun Ki (Harvard Medical School and Wellman Center for Photomedicine, Massachusetts General Hospital)

Most optical fibers are designed for the forward firing i.e. the light at the distal end of the fiber exit along the axis of the fiber. In some applications such as the laser surgery, printer, laser scanners, the side firing of the optical fiber is required. A few technologies including the polishing of the fiber tip or the assembly of micro-prism on the tip have been tried to implement the side firing of the fiber.In this paper, we present the microstructuring of optical fiber tip using the femtosecond laser for the multidirectional firing of the light. The femtosecond laser has been used widely for the microstructuring of various materials. We employed the femtosecond laser with the pulse duration of $185 \mathrm{fs}$ at the wavelength of $785 \mathrm{~nm}$ as the machining tool for the optical fiber. The distal end of the optical fiber with diameter of $125 \mathrm{~nm}$ was machined as the conical shape. The surface of the machined tip was irradiated with $\mathrm{CO} 2$ laser after femtosecond laser microstructuring. The $\mathrm{CO} 2$ laser irradiation leads to the melting and re-solidification of the fiber tip. That results in the smoothing of the micromachined conical shape. We were able to demonstrate the "multi-directional firing (or lateral firing)" using the modified optical fiber. It is expected that the multidirectional firing fiber can be applied in various medical and industrial applications.

\section{P 22: Solar Chemical Vapor Deposition of Graphene Flakes}

Presenter : Vitor BARANAUSKAS

Mr. LUNAZZI, Francisco (FEEC/UNICAMP) ; Dr. PETERLEVITZ, Alfredo (FEEC/UNICAMP) Co authors : Dr. CERAGIOLI, Helder José (FEEC/UNICAMP) ; Prof.BARANAUSKAS, Vitor (FEEC/UNICAMP) 
The purpose of this work is to show the use of solar energy in the Chemical Vapor Deposition (Solar-CVD) of carbon. A Fresnel lens of $40 \mathrm{~cm}$ diameter and $40 \mathrm{~cm}$ depth of focus was mounted on a moving frame to track the position of the sun in the sky and focus the light on the substrates that are immersed in the vapor, separated from the ambient atmosphere by a quartz window. The micro-sized carbon structures were synthesized on quartz or on polished Si substrates using ethanol as the carbon source, diluted in hydrogen (98\% vol.). A total flow rate of around $85 \mathrm{sccm}$, regulated by precision mass flow, and a total pressure of about $20 \mathrm{mBar}$, were maintained throughout. The deposition temperature was measured by a thermocouple fixed under the substrate. Optical and Scanning Electron Microscopy of the samples will be discussed along with results from Raman spectroscopic analyses of samples produced under different conditions.

\section{P 23: Raman Characterization of Nano crystalline Diamonds}

Presenter :. Vitor BARANAUSKAS

Dr. PETERLEVITZ, Alfredo Carlos (FEEC/UNICAMP) ; Dr. CERAGIOLI, Helder José (FEEC/UNICAMP)

Co-authors : Dr. TSUKADA, Jackson (FEEC/UNICAMP) ; Mr. GIOVANI ZANIN, Hudson

(FEEC/UNICAMP) ; Prof. BARANAUSKAS, Vitor (FEEC/UNICAMP)

Research on preparing and characterization of nano-structured carbon has been of high scientific and technological interest. The purpose of this work is to use Raman scattering to study the chemical bonds on the surface and grains of nanocrystalline diamonds. The samples were synthesized by hot-filament chemical vapor deposition (HFCVD) on polished Si substrates using ethanol as the carbon source, diluted in hydrogen (99.5\% vol.). Excitation lasers from infrared (735 nm) to ultraviolet $(325 \mathrm{~nm})$ have been used. Raman spectroscopic analyses will be discussed along with the Scanning Electron Microscopy (SEM) results.

\section{P 24: Pulsed laser deposition of gadolinia doped ceria layers at moderate temperature - a seeding approach}

Presenter : Katarzyna RODRIGO

Mrs. RODRIGO, Katarzyna (Fuel Cells and Solid State Chemistry Division, Risø DTU, Technical University Denmark, DK-4000 Roskilde, Denmark) Co-authors : Dr. HEIROTH, Sebastian (Paul Scherrer Institute, General Energy Research Department, CH-5232 Villigen PSI, Switzerland) ; Dr. PRYDS, Nini (Fuel Cells and olid State Chemistry Division, Risø DTU, Technical University of Denmark, DK-4000 Roskilde, Denmark) ; Dr. THEIL KUHN, Luise (Fuel Cells and Solid State Chemistry Division, Risø DTU, Technical University of Denmark, DK-4000 Roskilde, Denmark) ; Dr. ESPOSITIO, Vincenzo (Fuel Cells and Solid State Chemistry Division, Risø DTU, Technical University of Denmark, DK-4000 Roskilde, Denmark) ; Prof. LINDEROTH, Søren (Fuel Cells and Solid State Chemistry Division, Risø DTU, Technical University of Denmark, DK-4000 Roskilde, Denmark) ; Prof. SCHOU, Jørgen (Department of Photonics Engineering, Technical University of Denmark, DK-4000 Roskilde, Denmark) ; Dr. LIPPERT, Thomas (Paul Scherrer Institute, General Energy Research Department, CH-5232 Villigen PSI, Switzerland)

Ceria-based thin films are often applied as key functional components in miniaturized electroceramic devices such as solid oxide fuel cells or gas sensors. Processing routes that prevent thermal degradation and yield access to the optimum microstructures are sought. Multi-step growth, involving the preparation of ultrathin seed layers in the first stage of the deposition process is often envisaged to control the growth and physical properties of the subsequent coating. This work 
suggests that the limitations of conventional pulsed laser deposition (PLD), performed at moderate temperature $\left(400^{\circ} \mathrm{C}\right)$, to the growth of dense, gas impermeable $10 \mathrm{~mol} \%$ gadolinia-doped ceria (CGO10) solid electrolyte can be overcome by the seeding process. In order to evaluate the seed layer preparation, the effects of different thermal annealing treatments on the morphology, microstructure and surface roughness of ultrathin CGO10 layers with a thickness of $4 \mathrm{~nm}, 13 \mathrm{~nm}$ and $22 \mathrm{~nm}$, respectively, grown on $\mathrm{Mg}(100)$, were studied by atomic force microscopy and X-ray reflectometry.

\section{P 25: Evaluation of photocatalytic efficiency of several TiO2 type catalysts}

Presenter : Oldřich MACHALICKý

Mr. TOMICA, Marian (Institute of Organic Chemistry and Technology, Faculty of Chemical Technology, University of Pardubice) ; Dr. MACHALICKý, (Institute of Organic Chemistry and Technology, Faculty of Chemical Technology, University of Pardubice) ; Prof. HRDINA, Radim (Institute of Organic Chemistry and Technology, Faculty of Chemical Technology, University of Pardubice) ; Mr. VALENTA, Jiří (Precheza a.s.) ; Mr. KOVáŕ, Pavel (Precheza a.s.)

Determination of quantum efficiency of photochemical process is the one of key problems of photochemical kinetics. This quantity is well defined and experimentally available in the case of monomolecular photoreaction as a ratio of reaction rate and excitation rate. Quantum efficiency is constant during monomolecular photoreaction because the decrease of reaction rate follows the decrease of excitation rate in the course of reaction. In the case of heterogeneous photocatalysis, excitation rate does not decrease during reaction because of constant concentration of photocatalyst so that the efficiency of the process has to be expressed in a different way. In our work we have applied simplified kinetic description on photolysis of indigo carmine (as a standard model dye) using the set of several photocatalysts. Then we have evaluated kinetic data in accordance with our kinetic model and compared photocatalytic efficiencies of catalysts used. Photocatalytic efficiencies of several industrial photo-catalysts based on $\mathrm{TiO} 2$ were evaluated using photolysis of indigo-5,5'-disulfonic acid. Dispersions of $0.2 \mathrm{~g} / \mathrm{L}$ catalysts in the dye solution were irradiated with medium pressure mercury arc lamp Philips HPL-N $150 \mathrm{~W}$. Incident photon flux was in the range from 280 to $400 \mathrm{~nm}$. Photocatalytic efficiencies defined as a ratio of excitation rate and incident photon flux were within interval $0.10-0.48 \%$. The highest efficiency was observed for the catalyst Degussa P25.

\section{P 26: Oriented growth of Dion-Jacobson perovskite thin films on glass substrates under pulsed laser irradiation}

Presenter :. Tomohiko NAKAJIMA

Dr. NAKAJIMA, Tomohiko (National Institute of Advanced Industrial Science and Technology) Co-authors : Dr. TSUCHIYA, Tetsuo (National Institute of Advanced Industrial Science and Technology) ; Dr. KUMAGAI, Toshiya (National Institute of Advanced Industrial Science and Technology)

A new facile fabrication process for high quality uniaxial oriented grown Dion- Jacobson perovskite (DJP) RbLaNb2O7 thin films on amorphous substrates by means of an excimer laser assisted metal organic deposition (ELAMOD) has been investigated: the oriented crystal growth was occurred only by an excimer laser irradiation in air at $400{ }^{\circ} \mathrm{C}$ after a deposition of metal-organic 
solution and preheating at $400{ }^{\circ} \mathrm{C}$. The obtained $\mathrm{RbLaNb} 2 \mathrm{O} 7$ thin film showed perfect (010)-oriented growth on amorphous glass substrates owing to the gradient heating realized by pulsed laser irradiation, and the film surface had atomically flat terraces. We have confirmed that these characteristics of the obtained DJP RbLaNb2O7 thin films functioned very well as a seed layer for the fabrications of various perovskite oxide thin films with high orientation quality. For example, a conducting perovskite LaNiO3 film was also successfully grown on the seed layer with high (100)-orientation by means of the ELAMOD at $400{ }^{\circ} \mathrm{C}$. The obtained uniaxial oriented LaNiO3 showed very low resistivity, while the fabrication temperature was very low. In this presentation, we will report the detailed oriented growth mechanism of the DJP thin films, and the physical properties of the oriented grown perovskite films on this seed layer.

\section{P 27: Analysis of dispersion properties of rotational elliptic air hole photonic crystal fiber}

Presenter : Tzong-jer YANG

Prof. YANG, Tzong-jer (Department of Electrical Engineering, Chung Hua University) Co-authors : Prof. CHAU, Yuanfong (Ching Yun University) ; Mr. YEH, Han-hsuan (Vhing Yun University)

The two dimensional with a triangular-lattice cross sectional pattern of rotational elliptic air holes photonic crystal fiber (PCF) is investigated numerically by use of plan wave expansion (PWE) method. The statistical correlations between the dispersion and the various parameters are obtained. We compared the dispersion relations between the circular air holes PCF and the rotational elliptic one. We find that the rotational elliptic air holes PCF is better than that of circular one for the flatter dispersion curve and single mode in PCF. As an example, how to design the structure parameters for engineering the chromatic dispersion of rotational elliptic air holes PCF is demonstrated.

\section{P 28: On the size effect limit in line width of plasmon excitation of electrons at a aanoparticle surface}

Presenter : Dr. Elena S. APOSTOLOVA

Dr. APOSTOLOVA, Elena S. (Associate Professor, Department of Composite Materials,D.I. Mendeleev University of Chemical Technology of Russia) Co-authors : Prof. TIKHONOV, Anatoly P. (Head of Department, Department of Composite Materials,D.I. Mendeleev University of Chemical Technology of Russia)

Increase of the observed plasmon excitation with decrease of nanoparticle size iswell known. A method of estimation of radius of significant (Rsign) and of maximum(Rmax) appearance of size effect in properties of nanostate of a substance isformulated. The method is based on size effect in the melting temperature and onconception about boundary values of effect by change of property time e and $\mathrm{e}^{*} \mathrm{e}\left[{ }^{*}\right.$.Empirical dependence based on the vibration problem is preferable for evaluation of Rsign and Rmax. But due to deficit of observed size dependences of meltingtemperature of substances estimation of boundary rmaxK using the Kelvin equation issuggested:

$\mathrm{RmaxK}=\mathrm{sV} /(\mathrm{RT})$, there $\mathrm{T}$ is the melting temperature of bulk substance, $\mathrm{s}$ - surface tension at the $\mathrm{T}, \mathrm{V}$ - molar volume of the substance, $\mathrm{R}$ - the gas constant. The method was developed estimating RmaxK of elements and periodic dependence of RmaxK from the number is predicted. Change range of 
transition metals rmaxK is of $0.62-1.69 \mathrm{~nm}$.

* Apostolova E.S., Tikhonov A.P.// Rus. J. Coord. Chem. Received to Edition at 02/06/2010

\section{P 29: Pulsed Nd:YAG Laser Ablation of $\mathrm{Cu}, \mathrm{Ni}$, Mo and Si in Water}

Presenter :. Wee Ong SIEW

Mr. SIEW, Wee Ong (Multimedia University) Co-authors : Mr. NEE, Chen Hon (Multimedia University) ; Dr. YAP, Seong Shan (Norwegian University of Sci. \& Technol.) ; Dr. TOU, Teck Yong (Multimedia University)

Pulsed Nd:YAG laser with $355 \mathrm{~nm}$ and $10-\mathrm{Hz}$ output was used to ablate copper $(\mathrm{Cu})$, nickel (Ni), molybdenum (Mo) and silicon ( $\mathrm{Si}$ ) in water with laser fluence varying between $(1-10) \mathrm{J} / \mathrm{cm} 2$. The ablated materials which were dispersed in water were coated on highly polished silicon substrates and characterized by a scanning electron microscope. It was found that under the multiple-pass, scan-mode ablation over an area of $8 \times 5 \mathrm{~mm} 2$, ablated materials from these target, which included nanoparticles, would shield the laser beam [1] above the target surface, different structures, and nanoparticles. For Mo, its ablated materials could be transformed in ceramic-type of materials which might be partially soluble and few nanoparticles were found. For Si, owing to its relatively high optical absorption of the laser light at $355 \mathrm{~nm}$, hence the optical penetration, sub-surface melting was observed. For $\mathrm{Cu}$ ablation, nanoparticles of 100-200 nm were produced at laser fluence of $5 \mathrm{~J} / \mathrm{cm} 2$ but form coalesced into flakes. For $\mathrm{Ni}$, as compared to its high-vacuum ablation [2], laser-induced heating of suspended Ni materials in water turned into porous lumps. A study was also conducted on the threshold for nanoparticle generation with respect to the number of laser scans over the same ablation area.

References

[1] G. X. Chen, M. H. Hong, T. C. Chong, H.I. Elim, G.H. Ma, and W. Ji, J. Appl. Phys. 95, 1455, 2004.

[2] W.O. Siew, W.K Lee, H.Y.

\section{P 30: X-ray scattering based techniques for characterization of laser induced periodic surface structures on thin polymers films}

Presenter :. Marta CASTILLEJO

Dr. CASTILlEJO, Marta (Instituto de Química Física Rocasolano, Spanish National Research Council (CSIC)), Coauthors : Dr. REBOLLAR, Esther (Instituto de Química Física Rocasolano, Spanish National Research Council (CSIC)) ; Ms. PéREZ, Susana (Instituto de Química Física Rocasolano, Spanish National Research Council (CSIC)) ; Mr. HERNáNDEZ, Jaime J. (Instituto de Estructura de la Materia, Spanish National Research Council (CSIC)) ; Dr. RUEDA, Daniel (Instituto de Estructura de la Materia, Spanish National Research Council (CSIC)) ; Dr. EZQUERRA, Tiberio (Instituto de Estructura de la Materia, Spanish National Research Council (CSIC))

In the formation of laser induced periodic surface structures (LIPSS) the interference between the incoming and the surface scattered waves plays an important role, but the whole mechanism is still not fully understood. Various processes have been proposed for LIPSS formation in polymers such as changes in the surface tension in a melt region, migration of polymer chains, spatially modulated melting and crystallization, etc. In this work the influence of some polymer properties, i.e., 
crystallinity, glass transition and melting temperatures and light absorbance, on the LIPSS formation is studied. Films of poly (ethylene terephthalate) (PET), poly (trimethylene terephthalate) (PTT), polycarbonate (PC) and polyvinylidene fluoride (PVDF), prepared by spin-coating onto silicon wafers, were irradiated with $2 \mathrm{nd}, 4^{\text {th }}$ and 5 th harmonic of a polarized Nd:YAG laser (FWHM $6 \mathrm{~ns}, 10 \mathrm{~Hz}$ ). The surface topographies were observed by atomic force microscopy (AFM) and characterized structurally by grazing incidence small angle X-ray scattering (GISAXS), giving rise to well correlated results. GISAXS has been revealed as a powerful technique for the investigation of structures developed in very thin films since buried structures and larger surface areas are probed, which implies a much larger statistical significance compared to AFM. Additionally, grazing incidence wide angle X-ray scattering (GIWAXS) results give information on the crystallinity of the nanostructured films.

\section{P 31: Optical studies of chalcogenide (AsSe)100-x Sbx thin films}

Presenter : Tamara PETKOVA,

Dr. PETKOVA, Tamara (Institute of Electrochemistry and Energy Systems, Bulgarian Academy of Sciences) Co-authors : Mrs. ILCHEVA, Vania (Institute of Electrochemistry and Energy Systems, Bulgarian Academy of Sciences) ; Mr. PETKOV, Emil (University of Chemical Technology and Metallurgy) ; Dr. BOEV, Victor (Institute of Electrochemistry and Energy Systems, Bulgarian Academy of Sciences) ; Prof. PETKOV, Plamen (University of Chemical Technology and Metallurgy) ; Dr. SOKOL, Gabriel (National Institute for Lasers, Plasma and Radiations Physics) ; Prof. MIHAELSCU, Ion (National Institute for Lasers, Plasma and Radiations Physics)

Amorphous chalcogenides present a number of optical properties (good IR transparency, high linear and non-linear index of refraction, photoinduced changes of index of refraction, photoinduced shift of absorption edge), which make them promising materials for optical devices. We deposited thin arsenic-selenium-antimony films (AsSe)100-xSbx $(\mathrm{x}=0,5,10,15$ at.\%) on glass and silicon substrates by pulsed laser deposition (PLD) and vacuum thermal evaporation (VTE) from the corresponding bulk materials. We demonstrated the possibility to deposit by both methods high-quality films with appropriate optical parameters in view of potential applications in optics. The distribution of the optical parameters of the VTE- and PLD- layers as a function of deposition technique and composition was studied. The optical constants of the films have been estimated by Swanepoel method. The optical band-gap was derived from the spectra with the Tauc procedure. The variation of the optical band-gap was discussed in terms of the local ordering modification due to Sb incorporation.

\section{P 32: Harmonic generation in ablation plumes of wide bandgap semiconductors}

Presenter : Dr. Marta CASTILLEJO

Dr. CASTILLEJO, Marta (Instituto de Química Física Rocasolano, Spanish National Research Council (CSIC)) Co-authors : Ms. LóPEZ-ARIAS, Maria Encina (Instituto de Química Física Rocasolano, Spanish National Research Council (CSIC)) ; Dr. SANZ, Mikel (Instituto de Química Física Rocasolano, Spanish National Research Council (CSIC)) ; Dr. OUJJA, Mohamed (Instituto de Química Física Rocasolano, Spanish National Research Council (CSIC)) ; Dr. DE NALDA, Rebeca (Instituto de Química Física Rocasolano, Spanish National Research Council (CSIC))

Low-order harmonics (3rd at $355 \mathrm{~nm}$ and 5 th at $213 \mathrm{~nm}$ ) of the fundamental laser radiation of a $\mathrm{Nd}$ :YAG laser (1064 nm, $15 \mathrm{~ns}$ ) have been produced in ablation plumes of the wide bandgap II-VI semiconductors $\mathrm{CdS}$ and $\mathrm{ZnS}$, where ablation is triggered by a second $\mathrm{Nd}: \mathrm{YAG}$ laser operating at 
either 532 or $266 \mathrm{~nm}$. The fundamental laser beam propagates parallel to the surface of the target at variable distance, with delays with respect to the ablating laser in the $0-40 \mu$ s range. Together with harmonic generation ( $\mathrm{HG})$, the in situ optical diagnosis of the plume reveals spontaneous emissions of atomic $\mathrm{Cd}$ and $\mathrm{Zn}$ species in the respective target plumes. A second diagnosis of the plume is provided by scanning electron microscopy analysis of the material deposited on a Si substrate placed in front of the target. This analysis reveals the presence of nanoaggregates with sizes \&1t; $100 \mathrm{~nm}$. The spatiotemporal behaviour of the generated harmonics indicates two types of plume populations contributing sequentially to the overall $\mathrm{HG}$ signal. It is found that atomic species are mostly responsible of $\mathrm{HG}$ at early times (\&lt; $500 \mathrm{~ns}$ ), while the nanoaggregates contribute at longer times. These results permit us to propose a new methodology to wholly explore spatially and temporally the expanding ablation plume, and to advance the knowledge on HG processes in laser plasmas [Ganeev, J. Phys. B 40, R213, 2007; Oujja, de Nalda, López-Arias, Torres, Marangos, Castillejo, Phys. Rev. A 81, 043841, 2010$].$

\section{P 33: Investigation of resonant peaks in the symmetric and asymmetric multilayer narrowband transmission filters}

Presenter : Chien-jang WU

Prof. WU, Chien-jang (National Taiwan Normal University) Co-authors : Prof. CHANG, Tsung-wen (Chang Gung University) ; Mr. WANG, Zheng-hui (National Taiwan Normal University) ; Prof. YANG, Tzong-jer (Chung Hua University)

The resonant transmission peaks in the asymmetric and symmetric multilayer narrowband transmission filters are theoretically investigated based on the transfer matrix method. In an asymmetric filter there exists only one resonant peak within the photonic band gap and its position can be changed when the design wavelength is varied. In a symmetric filter, it is found that there are two resonant peaks. Using Bloch wave approximation, an investigation of these two resonant peaks has been made and they are shown to correspond to the symmetric and the antisymmetric field solutions in the defect layer.

\section{P 34: Effects of losses on the transmission and reflection in the single-negative materials}

Presenter : Chien-jang WU

Prof. WU, Chien-jang (National Taiwan Normal University) Co-authors : Mr. LIN, Wei-hsiao (National Cheng-Kung University) ; Prof. CHANG, Shoou-jinn (National Cheng-Kung University)

In this work, we investigate the angular dependence of transmission and reflection in the singlenegative (SNG) materials. We consider a model structure of an SNG bilayer consisting of epsilonnegative $(\mathrm{ENG})$ and mu-negative $(\mathrm{MNG})$ layers. The wave transmission and reflection properties due to the losses from the ENG and MNG materials are specifically investigated. With the presence of losses in the ENG and MNG materials, some unusual wave properties will be explored and numerically demonstrated. 


\section{P 35: Laser processing of ormosils for tissue engineering applications}

Presenter : Andreea MATEI

Dr. MATEI, Andreea (Petru Poni Institute of Macromolecular Chemistry, Grigore Ghica Voda 41A, Iasi, Romania; National Institute for Lasers, Plasma and Radiation Physics, Atomistilor 409, 76900 Bucharest-Magurele, Romania) Coauthors : Dr. ZAMFIRESCU, Marian (National Institute for Lasers, Plasma and Radiation Physics, Atomistilor 409, 76900 Bucharest-Magurele, Romania) ; Dr. DINESCU, Maria (National Institute for Lasers, Plasma and Radiation Physics, Atomistilor 409, 76900 Bucharest-Magurele, Romania) ; Dr. BURUIANA, Emil (Petru Poni Institute of Macromolecular Chemistry, Grigore Ghica Voda 41A, Iasi, Romania) ; Dr. BURUIANA, Tinca (Petru Poni Institute of Macromolecular Chemistry, Grigore Ghica Voda 41A, 6600 Iasi, Romania) ; Mrs. SIMA, Livia (Institute of Biochemistry, Romanian Academy, Splaiul Independentei 296, 060031, Bucharest 17, Romania) ; Dr. PETRESCU, Stefana (Institute of Biochemistry, Romanian Academy, Institute of Biochemistry, Romanian Academy, Splaiul Independentei 296, 060031, Bucharest 17, Romania)

Hybrid methacrylates based on silane derivates (ormosils) can find multiple applications in electronics, microtechnology, corrosion resistant coatings, dentistry and biomedical implants. Unique properties can appear as a result of the presence of both inorganic chains responsible for chemical and thermal stability, hardness, transparency, and organic groups, which bring new advantages such as the possibility of functionalization, easily low temperature processing etc. 2D and 3D structures of hybrid polymers were produced by Two Photon Polymerization (2PP). A Ti: Sapphire laser with $200 \mathrm{fs}$ pulse duration and $2 \mathrm{kHz}$ repetition rate, emitting at $775 \mathrm{~nm}$ was used for the $2 \mathrm{PP}$ experiments. The obtained structures (scaffolds) were tested in different cell cultures for further applications in tissue engineering. Cells morphology, adhesion and alignment were studied on polymeric structures with different shape obtained in various experimental conditions. Primary human dermal fibroblasts (FBD), normal human epidermal melanocytes (NHEM) and dysplastic oral keratinocytes (DOK) were investigated with the aim of obtaining a dermal graft.

\section{P 36: Spacer tuned room temperature magneto resistance in all manganite superlattices}

Presenter : Jauyn Grace LIN

Dr. LIN, Jauyn Grace (Center for Condensed Matter Sciences, National Taiwan University)

In this study, the spacer-thickness dependent room-temperature- magnetoresistance(RTMR) is investigated in ferromagnetic/antiferromagnetic (FM/AFM) superlattices composed of ten repetition of $\mathrm{La} 0.7 \mathrm{Sr} 0.3 \mathrm{MnO} 3 / \mathrm{Nd} 0.6 \mathrm{Ca} 0.4 \mathrm{MnO} 3$ [LSMO/NCMO]10. The series of superlattices sample are fabricated with the method of Pulsed Laser Deposition. The thickness of the FM LSMO layer is fixed at $5 \mathrm{~nm}$; while that of the spacer NCMO-layer varies from 0 to $5 \mathrm{~nm}$. RTMR is measured with varying the field from zero to $10 \mathrm{kOe}$ with field applied at two directions, in plane (IP) and out-of-plane (OOP). It's observed that the magnitude of RTMR decreases near linearly with increasing the applied field. At $10 \mathrm{kOe}, \mathrm{RTMR}$ oscillates with increasing the spacer-thickness of NCMO at both IP and OOP configurations. The MR anisotropy mainly occurs at low field, which is associated with the difference in their directional coercive field. Interestingly, RTMR and the saturation magnetization (MS) show no correlation, which implies an un-conventional mechanism governing the magneto transport properties in these all manganite super lattices. 


\section{P 37: Epitaxial growth of atomically flat gadolinia-doped ceria thin films by pulsed laser deposition}

Presenter : Yunzhong CHEN

Dr. CHEN, Yunzhong (Fuel Cells and Solid State Chemistry Division, Risø DTU) Co-authors : Dr. PRYDS, Nini (Fuel Cells and Solid State Chemistry Division, Risø DTU) ; Prof. SCHOU, Jørgen (Department of Photonics Engineering, Technical University of Denmark) ; Prof. LINDEROTH, Søren (Fuel Cells and Solid State Chemistry Division, Risø DTU)

The epitaxial growth of gadolinium-doped ceria (Ce0.8Gd0.2O2, CGO) films on (001) TiO2terminated SrTiO3 (STO) substrates using pulsed laser deposition has been investigated by in situ reflective high energy electron diffraction (RHEED). The initial film growth shows a StranskyKrastanov growth mode, with three dimensional (3D) islanding after a critical thickness of 2 monolayers. Interestingly, this 3D film growth is replaced by a two-dimensional (2D) island nucleation during the subsequent film deposition, which results in an atomically flat surface of the deposited films. Detailed analyses of RHEED patterns and the XRD results both show that the CGO films are epitaxially grown in (001) orientation with a $45^{\circ}$ cell rotation to accommodate half of the diagonal of the CGO fluorite unit cell $(\mathrm{aCGO}=0.5420 \mathrm{~nm})$ to the STO perovskite unit cell $(\mathrm{aSTO}=0.3905 \mathrm{~nm})$. The atomic force microscopy (AFM) measurements confirmed the atomically smooth surface, which shows a root mean roughness of $0.4 \mathrm{~nm}$. The flat CGO film will be an attractive electrolyte material for micro solid oxide fuel cell operating at low temperature.

\section{P 38: Surface foaming of thin collagen films induced by pulse width variable femtosecond laser pulses}

Presenter: Albena DASKALOVA

Dr. DASKALOVA, Albena (Institute of Electronics, Bulgarian Academy of Sciences) Co-authors : Dr. SELIMIS, Alexandros (Institute of Electronic Structure and Laser (IESL), Foundation of Research and Technology-Hellas (FORTH), P.O. Box 1527, 71110 Heraklion, Crete, Greece) ; Dr. MANOUSAKI, Alexandra (Institute of Electronic Structure and Laser (IESL), Foundation of Research and Technology-Hellas (FORTH), P.O. Box 1527, 71110 Heraklion, Crete, Greece, Department of Physics, University of Crete, Voutes Campus 71003, Heraklion, Crete, Greece) ; Dr. GRAY, David (Institute of Electronic Structure and Laser (IESL), Foundation of Research and Technology-Hellas (FORTH), P.O. Box 1527, 71110 Heraklion, Crete, Greece) ; Dr. RANELLA, Anthi (Institute of Electronic Structure and Laser (IESL), Foundation of Research and Technology-Hellas (FORTH), P.O. Box 1527, 71110 Heraklion, Crete, Greece) ; Prof. FOTAKIS, Costas (Institute of Electronic Structure and Laser (IESL), Foundation of Research and Technology-Hellas (FORTH), P.O. Box 1527, 71110 Heraklion, Crete, Greece,Department of Physics, University of Crete, Voutes Campus 71003, Heraklion, Crete, Greece)

The ability to produce idealized cellular constructs is essential for understanding and controlling intercellular processes and ultimately for producing engineered tissue replacements. Preliminary results have been obtained on collagen modification by irradiation with single and multiple pulses of femtosecond laser with variable pulse duration. Irradiation of collagen thin film with single pulses of femtosecond duration results in creation of foam layer with micrometer thickness. The structure and thickness of the layer strongly depends on the number of the applied laser pulses. The surface 
properties of collagen thin films before and after Ti-sapphire irradiation with $800 \mathrm{~nm}$ were investigated by means of the technique Field Emission Scanning Electron Microscope (FESEM). Examination of the interaction of ultra-short laser pulses with collagen films is useful for controlling the chemical and microstructural modification of the created foam layer.

\section{P 39: Optical Properties of Samarium (II) complexes with crown ethers}

Presenter : Mitsuhiro KUSABA

Prof. KUSABA, Mitsuhiro (Department of Electronics, Information and Communication Engineering, Osaka Sangyo University) Co-authors : Ms. YAMADE, Eriko (Department of Chemistry, Graduate School of Science, Osaka City University) ; Mr. NISHIDA, Daisuke (Department of Chemistry, Graduate School of Science, Osaka City University) ; Dr. YATSUHASHI, Tomoyuki (Department of Chemistry, Graduate School of Science, Osaka City University) ; Prof. NAKASHIMA, Nobuaki (Department of Chemistry, Graduate School of Science, Osaka City University) ; Prof. TSUNAWAKI, Yoshiaki (Department of Electronics, Information and Communication Engineering, Osaka Sangyo University)

Photoredox reaction of rare earths ions has stimulated much interest because of their potential use in areas such as optoelectronic devices. Various studies on three-dimensional optical memory using the photo reduction of some rare earths ions such as $\mathrm{Eu} 3+$ and $\mathrm{Sm} 3+$ in glass have been demonstrated with femtosecond laser irradiation. However, the exact mechanism of the photo reduction has yet to be identified. In order to clarify the mechanism, the optical properties of the photoproduct, Eu2+ and $\mathrm{Sm} 2+$ have to found. Although the optical properties of Eu2+ have been investigated, there was no report on that of $\mathrm{Sm} 2+$. In this paper, optical properties of $\mathrm{Sm} 2+$ complexes with crown ethers in methanol solution were studied. The samples of Sm2+ were prepared by photoreducing Sm3+ to Sm2+ in the degassed methanol solution which contained $\mathrm{SmCl3}$ and crown ethers, such as 12-C-4, 15-C-5, and 18-C6. Absorption and fluorescence spectra of $\mathrm{Sm} 2+$ samples were measured using a spectrophotometer and spectrofluorometer, respectively. The lifetime of $\mathrm{Sm} 2+$ fluorescence was determined by measuring the time profile of the fluorescence intensity after a N2 laser irradiation. The lifetime of Sm2+ samples with 12-C-4, 15-C5 and 18-C6 were estimated to be $4.5 \mathrm{~ns}, 4.1$ microsecond and $15 \mathrm{~ns}$, respectively. The fluorescence yields of Sm2+ with 15-C5 and 18-C-6 were 0.047 and 0.00083 , respectively.

\section{P 40: Computer-generated holograms written directly on a silicon surface including 3-D effects}

Presenter : Kristian Juncher WæDEGAARD

WÆDEGAARD, Kristian Juncher (Department of Physics and Astronomy, Aarhus University) Co-authors : BALLING, Peter (Department of Physics and Astronomy, Aarhus University)

3-D effects have been implemented in directly written computer-generated holograms. An infrared femtosecond laser (795 nm, $100 \mathrm{fs)} \mathrm{was} \mathrm{used} \mathrm{to} \mathrm{write} \mathrm{the} \mathrm{holographic} \mathrm{bit} \mathrm{pattern} \mathrm{on} \mathrm{a} \mathrm{silicon} \mathrm{surface} \mathrm{by}$ selective ablation. The sample required no pre- or post-treatment and the calculation of the hologram included focusing, so that no lenses were needed to reconstruct the holographic image. Using low pulse energies $(\sim 100 \mathrm{~nJ})$ and a high NA lens $(\mathrm{f}=10 \mathrm{~mm}$, NA 0.545$)$, distances between pixels of down 
to $3 \mu \mathrm{m}$ corresponding to a resolution of $\sim 8500 \mathrm{dpi}$, was achieved. The high resolution has several advantages; it improves the sharpness of the holographic image and increases the distance between different diffraction orders, which enables production of larger images without overlapping. However, a higher resolution does increase the production time. The 3-D effects were incorporated by assigning different depths to different parts of the object from which the hologram was calculated. When viewed on-axis this effect does not show, but when moved off-axis the encoded depth causes the different parts of the holographic image to move relative to each other. In contrast to usual production methods based on lithography, the femtosecond-laser writing method provides a quick way to make unique holograms with possible applications in e.g. anti counterfeiting and optical keys. The incorporation of 3-D effects in CGHs would further increase the level of security for such applications.

\section{ID 41: Femtosecond laser ablation rates of dielectric materials: Experiments and modeling}

Presenter : Kristian Juncher WæDEGAARD

WæDEGAARD, Kristian Juncher (Department of Physics and Astronomy, Aarhus University) Co-authors : CHRISTENSEN, Mehrnaz Nesari (Department of Physics and Astronomy, Aarhus University) ; CHRISTENSEN, Bjarke Holl (Danish Technological Institute, Aarhus); LE, Dang Quang Svend (Interdisciplinary Nanoscience Center, iNano, Aarhus University) ; BYSKOV-NIELSEN, Jeppe (Department of Physics and Astronomy, Aarhus University) ; BALLING, Peter (Department of Physics and Astronomy, Aarhus University)

Short-pulse laser ablation of dielectrics is modeled using multiple-rate equations(MRE) for the electronic excitation combined with a one-dimensional description of the optical propagation into the material. In the MRE model, the generation of conduction-band electrons is initiated by strong-field excitation and followed by multiplication through impact ionization from energetic electrons heated by inverse bremsstrahlung. The inclusion of light propagation in the model allows for a determination of ablation depths. The chosen criterion for ablation is that a certain percentage of the electrons are in the conduction band. Simulations based on this model show that for shorter wavelength or lower band gap, the ablation threshold is lower but the ablation depth is smaller. In both cases, the lower ablation threshold is explained by a reduction in the order of the nonlinear excitation process. This facilitates multiphoton processes, which generates seed electrons and thereby initiates ablation at a lower fluence. Since the multiphoton excitation starts rapidly, the electron density in the outermost layers increases quickly, which increases the absorption. This results in a short optical penetration depth, which explains the decrease in ablation depth. The model is compared with single-shot experiments done on well-defined single-crystal dielectric samples. The ablated holes are analyzed by SEM and AFM and theresults fit the model well when adapting an ablation criteria of $10 \%$.

\section{$P$ 42: Shielding effect of laser-induced plasma in glass: Temporal behavior of nitrogen and analyte emission lines}

Presenter : Dong Hyoung LEE

Mr. LEE, Dong Hyoung (KAIST) Co-authors : Mr. KIM, Tae Hyeong (KAIST) ; Dr. JUNG, Euo Chang (Korea Atomic Energy Research Institute) ; Prof. YUN, Jong-il (KAIST) 
A characteristic of the plasma shielding effect was investigated through simultaneous temporal behaviours of nitrogen emission in ambient air and analyte-specific emission in a glass matrix with varying lens-to-sample distance (LTSD) and laser pulse energy. Plasma was generated by focusing a third harmonics of Nd:YAG laser at $355 \mathrm{~nm}$ onto glass samples. Temporal behaviors of the nitrogen emission were correlated reversely with a fluctuation of the temporal behaviour of various analyte emissions in the glass samples. At focal point, the values of the emission intensity of calcium for the late part of the laser shot numbers were around 3 times higher than those for the beginning part, whereas the values at defocused condition did not differ much. The efficiency of laser ablation is strongly combined with laser irradiance arriving at the sample surface due to laser energy absorption by air plasma. During around 50 laser shots, an increase in the laser pulse energy had no significant impact on the intensities of the calcium emission lines. However, the nitrogen emission intensities were considerably affected by varying the laser pulse energy. Based on the reverse relation between the intensities of nitrogen and calcium emission intensities, the corrected values of the calcium emission line were calculated. This methodology shows consistent results independent of experimental conditions such as different LTSD and laser energies.

\title{
P 43: Force generation through low-power laser-metal interaction
}

\author{
Presenter : Hideyuki HORISAWA
}

Prof. HORISAWA, Hideyuki (Department of Aeronautics and Astronautics, Tokai University) Co-authors : Prof. FUNAKI, Ikkoh (Japan Aerospace Exploration Agency)

The micro-Newton thrust generation was observed through low-power continuous-wave laser and aluminum foil interaction without any remarkable ablation of the target surface. To evaluate the thrust characteristics, a torsion-balance thrust stand capable for the measurement of the thrust level down to micro-Newton ranges was developed. In the case of an aluminum foil target with 10 micrometer thickness, the maximum thrust level was 15 micro-Newtons when the laser power was 20 W. It was also found that the laser intensity, or laser power per unit area, irradiated on the target was significantly important on the control of the thrust even under the low- intensity level.

\section{P 44: Forward plasma emission through laser-foil interaction with nano-second lasers}

Presenter : Prof. Hideyuki HORISAWA

Prof. HORISAWA, Hideyuki (Department of Aeronautics and Astronautics, Tokai University) Co-authors: Prof. YAMAGUCHI, Shigeru (Department of Physics, Tokai University)

Fundamental investigations on plasma diagnostics of a forward laser plasma emission employing laserfoil interactions were conducted for an Al-foil target irradiated with an Nd:YAG laser of 1J/pulse with pulse-width of 10 nsec. A time-of-flight measurement was also conducted to evaluate ion speeds. In addition, temporal evolutions of electron temperatures and densities were evaluated with electrostatic probes and spectroscopic diagnostics. From the results, it was shown that a speed of ions in a forward direction were about $135 \mathrm{~km} / \mathrm{sec}$. Also it was shown that the plasma temperature and density were about $2.5 \sim 3 \mathrm{eV}$ and $10 \mathrm{e} 10 \mathrm{~cm}^{\wedge}-3$. 


\section{P 45: Comparison of photon rocket and laser-plasma accelerator for space propulsion applications}

Presenter : Prof. Hideyuki HORISAWA

Prof. HORISAWA, Hideyuki (Department of Aeronautics and Astronautics, Tokai University) Co-authors: Prof. KIMURA, Itsuro (University of Tokyo, Prof. Emeritus)

Characteristics of compact laser plasma accelerators utilizing high-power laser and thin-target interaction were reviewed as a potential candidate of future spacecraft thrusters capable of generating relativistic plasma beams for interstellar missions. Based on the special theory of relativity, motion of the relativistic plasma beam exhausted from the thruster was formulated. Relationships of thrust, specific impulse, input power and momentum coupling coefficient for the relativistic plasma thruster were derived. It was shown that under relativistic conditions, the thrust could be extremely large even with a small amount of propellant flow rate. Moreover, it was shown that for a given value of input power thrust tended to approach the value of the photon rocket under the relativistic conditions regardless of the propellant flow rate.

\section{$P$ 46: Luminescence properties and deposition condition of $\mathrm{Mn} 2+$-doped $\mathrm{Zn}_{2} \mathrm{SiO}_{4}$ thin film phosphors by pulsed laser deposition}

Presenter : Hyun Kyoung YANG

Prof. JEONG, Jung Hyun (Pukyong National University); Dr. YANG, Hyun Kyoung (Pukyong National University) Co-authors : Mr. CHUNG, Jong Won (Pukyong National University) ; Prof. MOON, Byung Kee (Pukyong National University) ; Prof. CHOI, Byung Chun (Pukyong National University) ; Prof. LEE, Ho Sueb (Changwon National University)

Mn-doped $\mathrm{Zn} 2 \mathrm{SiO} 4$ is a well-known green phosphor for its high luminescent efficiency and chemical stability. Zn2SiO4:Mn2+ phosphor is widely used in cathode ray tubes, plasma display panels, and lamps as a green phosphor. $\mathrm{Zn} 2 \mathrm{SiO} 4: \mathrm{Mn} 2+$ films were grown using pulsed laser deposition technique on A12O3 (0001) substrates under different substrate temperatures and oxygen pressures. The crystalline phase and surface morphology of the films were very dependent on the oxygen pressure and substrate temperature and they affected the luminescent brightness of the films. The crystalline structure and microstructure of these films have been characterized by X-ray diffraction and electron microscopy and their luminescent properties have been evaluated at room temperature using a luminescence spectrometer and excitation by a broadband incoherent ultraviolet light source. The emitted radiation was dominated by the red emission peak at $523 \mathrm{~nm}$ radiated from the transition of 4T1-4A1 of Mn2+ ions. 


\section{P 47: Photoluminescent Property and Energy Transfer from Host Lattice to Eu3+ in Y6WxMo(1- x)O12:Eu Nanocrystalline}

Presenter : Dr. Huaiyong LI

Prof. JEONG, Jung Hyun (Pukyong National University) ; Dr. LI, Huaiyong (Pukyong National University) Coauthors : Dr. YANG, Hyun Kyoung (Pukyong National University) ; Prof. JANG, Kiwan (Changwon National University) ; Prof. LI, Ho Sueb (Changwon National University)

The title compounds were synthesized by using sol-gel method, followed by annealing at gradually increased temperature. The powders crystallize in cubic or rhombohedral phase depending on the constitution and the calcination temperature. For a given system, powders exhibit systematically variation in the structure and photo luminescent properties with the annealing temperature. While for different systems, they show attractive similarity and diversity in the evolution. Near UV or violet light can be absorbed by the W-O or Mo-O group in the lattice hosts, and then the energy is transferred to the Eu3+, resulting in red light emission. The energy transfer in the systems was discussed. For the ending compounds, energy transfer from W-O group is far more efficient than from Mo-O group to $\mathrm{Eu} 3+$, but in the solid solution, the $\mathrm{W}-\mathrm{O}$ group excitation is almost entirely quenched by the Mo-O group, meantime, the energy transfer efficiency from Mo-O to Eu3+ is greatly enhanced.

\section{P 48: Synthesis and up-conversion luminescence properties of Y2O3:Yb3+/Tm3+ nanophosrphors}

Presenter : Jong Won CHUNG

Prof. JEONG, Jung Hyun (Pukyong National University) ; Mr. CHUNG, Jong Won (Pukyong National University) Co-authors : Prof. MOON, Byung Kee (Pukyong National University) ; Prof. CHOI, Byung Chun (Pukyong National University)

Especially, lanthanide-doped luminescent nanocrystals display interesting optical characteristics, which are poised to be exploited in the development of displays and phosphor application. This is largely due to their unique intra $4 \mathrm{f}$ transitions, which are less influenced by the ligand field (due to the shielding of the $4 \mathrm{f}$ orbitals by the outer $5 \mathrm{~s}$ and $5 \mathrm{p}$ orbitals) leading to sharp emissions. In addition, the lanthanide ions $(\mathrm{Ln} 3+)$ possess several electronic states at various energies, which afford them the ability to absorb one or more low-energy near-infrared (NIR) photons and subsequently convert them to highenergy emissions. Y2O3:Yb3+/Tm3 + nanophosphors were prepared by the solvothermal reaction method. And the samples sintered in air at $900 \mathrm{oC}$ for $3 \mathrm{~h}$. The effects of the crystalline structures, surface morphologies and luminescence spectra were investigated by using XRD, SEM and PL. The purpose of this study is to compare the luminescence characteristics of $\mathrm{Y} 2-\mathrm{xO} 3: \operatorname{Tm} 3+\mathrm{x}(\mathrm{x}=1,2,3$ and $4 \mathrm{~mol} \%)$ and $\mathrm{Yb} 3+(10 \mathrm{~mol} \%)$ phosphors which were synthesized various $\mathrm{Tm} 3+$ ion concentration. One typical ultraviolet up-conversion luminescence lines positioned at $362 \mathrm{~nm}$ was found, and can be attributed to the two-photon luminescence transition of 1D2 3H6. The visible up-conversion luminescence lines at $451 \mathrm{~nm}$ and $478 \mathrm{~nm}$ were found also, which result from the photon luminescence transitions of 1D2 3F4 and $1 \mathrm{G} 4 \quad 3 \mathrm{~h} 6$ of Tm3+ ion, respectively. 


\title{
P 49: Optical properties and ambient oxygen effects of Li-doped ZnO thin films by PLD method
}

\author{
Presenter : Saes Byul KIM
}

Prof. CHOI, Byung Chun (Pukyong National University) ; Mrs. KIM, Saes Byul (Pukyong National University) Coauthors : Mr. LI, Guojie (Pukyong National University) ; Prof. JEONG, Jung Hyun (Pukyong National University) ; Prof. LEE, Ho Sueb (Changwon National University)

We studied the effects of $\mathrm{Li}$ incorporation to physical properties of $\mathrm{ZnO}$ thin film, which is comparable with the native defects in the $\mathrm{ZnO}$. The $\mathrm{Li}$ doped $\mathrm{ZnO}$ (ZnO:Li) thin films were grown on Al2O3(0001) and $\mathrm{Pt}(111) / \mathrm{Ti} / \mathrm{SiO} 2 / \mathrm{Si}(100)$ substrate by using the pulsed laser deposition(PLD) method. The targets were employed as high purity $\mathrm{ZnO}$ ceramics with different Li contents, $\mathrm{Li} 0.05$ at.\%, Li2O $0.05 \mathrm{~mol} \%$, Li 0.10 at.\% and $\mathrm{Li} 0.15$ at.\%. We observed that the optical and electrical properties of $\mathrm{ZnO}: \mathrm{Li}$ thin films depend on the Li concentration. The crystalline quality and surface morphology of thin films were studied by using the X-ray diffractions and scanning probe microscope images. The thicknesses of $\mathrm{ZnO}: \mathrm{Li}$ thin films were estimated by the interference patterns of optical transmittance data. The surface of $\mathrm{ZnO}: \mathrm{Li}$ thin films was featured with sub-micron sized grains. The grains seemed to be aligned along the c-direction and wereconfirmed by the X-ray diffractions. The in-situ annealing was performed under dilute $\mathrm{O} 2$ gas atmosphere in order to investigate the $\mathrm{Li}$ doping on $\mathrm{ZnO}: \mathrm{Li}$. In order to investigate the potential transparent conductive application of $\mathrm{ZnO}: \mathrm{Li}$ thin films, photoluminescence and Hall conductivity were discussed. The Hall coefficient and carrier density were weakly varied with the in-situ annealing temperature. Consequently, it is expected that $\mathrm{ZnO}: \mathrm{Li}$ thin films were almost epitaxially grown on Al2O3(0001) substrate and exhibits n-type conductivity at room temperature.

\section{P 50: Micro-mechanical interlocking by laser surface structuring}

Presenter :. Peter BALLING

Mr. BYSKOV-NIELSEN, Jeppe (Department of Physics and Astronomy, Aarhus University) ; Dr. BALLING Peter (Department of Physics and Astronomy, Aarhus University)

Laser surface structuring provides the ability to generate new strong composite materials by employing micro-mechanical interlocking (MMI). It is demonstrated that at optimized laser-processing parameters, a high-average power nanosecond laser is able to undertake precise micro-structuring of large surface areas with volume removal rates approaching $1 \mathrm{~mm}^{\wedge} 3 / \mathrm{s}$, thereby making it an industrial relevant process. Interlocking strength between metal and plastic in the kilo Newton regime is obtained by structuring only $2 \mathrm{~mm} \times 5 \mathrm{~mm}$ of the metal surface and subsequently applying the plastic in an injection molding process. Theoretical calculations of the strength are shown to be in excellent agreement with the experimental data. Finally the permeability and corrosion resistance of the laser structured MMI joints have been investigated. We show that the permeability of the interconnect is consistent with the Hagen-Poiseuille equation and independent on the precise laser structuring geometry. The corrosion resistance of the samples is greatly reduced when laser structuring is done in ambient atmospheric air. The reduction is less pronounced when samples are structured in an inert argon atmosphere but the corrosion resistance is still significantly lower than for the base material. 


\title{
$P$ 51: Comparison between $p-6 P$ thin films and nano fibers on transistor platforms
}

\author{
Presenter :. Luciana TAVARES
}

Ms. TAVARES, Luciana (University of Southern Denmark) Co-authors : Dr. KJELSTRUP-HANSEN, Jakob (University of Southern Denmark) ; Mr. THILSING-HANSEN, Kasper (University of Southern Denmark) ; Prof. RUBAHN, Horst-günter (University of Southern Denmark)

The organic semiconductor para-hexaphenylene (p-6P) can be used as the light-emitting material in organic light-emitting field-effect transistors (OLEFETs), has a low cost, and is easy to process which could allow, for example, the development of mechanically flexible optoelectronics. In this work, a systematic investigation was made of the electrical properties of $\mathrm{p}-6 \mathrm{P}$ both in the form of an evaporated thin film and also in the form of nanofibers that were transferred from a template substrate (muscovite mica) onto the transistor platform. We have investigated both bottom contact, bottom gate (BC/BG) and top contact, bottom gate (TC/BG) configurations, both with gold electrodes. Thin films and nanofibers measurements showed the top contacts provide higher current (more than two orders of magnitude) than similar bottom contacts. It could be explained by the fact that for top contacts the area over which the carrier injection occurs in significantly larger compared to the bottom contact geometry.

\section{$P$ 52: Visualization and measurement of the near field enhancement of gold nano triangles by two-photon polymerization}

Presenter : Tobias GELDHAUSER

Dr. GELDHAUSER, Tobias (University of Hokkaido, Research Institute for Electronic Science) Co-authors : Mr. IKEGAYA, Shintarou (University of Hokkaido, Research Institute for Electronic Science) ; Mr. KOLLOCH, Andreas (University of Konstanz, Konstanz, Germany) ; Dr. MURAZAWA, Naoki (University of Hokkaido, Research Institute for Electronic Science) ; Prof. UENO, Kosei (Research Institute for Electronic Science, Hokkaido University, Sapporo, Japan / PRESTO, Japan Science and Technology Agency, Kawaguchi, Japan) ; Prof. BONEBERG, Johannes (University of Konstanz, Konstanz, Germany) ; Prof. LEIDERER, Paul (University of Konstanz, Konstanz, Germany) ; Prof. SCHEER, Elke (University of Konstanz, Konstanz, Germany) ; Prof. MISAWA, Hiroaki (Research Institute for Electronic Science, Hokkaido University, Sapporo, Japan)

The field of plasmonics offers a wide range of different applications where specially tailored structures are needed and it is important to understand the shape and strength of the near field of nanostructures. We compare the experimentally obtained near field of nanotriangles with finite difference in the time domain (FDTD) simulations in order to investigate the influence of the polarization of the light field with respect to the orientation of the triangles and quantitatively and qualitatively verify the results given by FDTD simulations. As experimental technique to probe the near field we use the method of two-photon polymerization (TPP). The link between experiment and theory is established by the extinction spectra of the nanostructures.

The near field of gold triangles is visualized for structures with a side length of $540 \mathrm{~nm}$ and 85 $\mathrm{nm}$ excited by a $800 \mathrm{~nm}$ fs laser pulse. In case of the smaller triangle, the near field enhancement is parallel to the polarization of the laser light in consistency with a merely dipolar plasmonic excitation. These findings change for the near field of the bigger triangles. Here the maxima of the near field are rotated by 90 degree with respect to the polarization of the laser orthogonal to the base of the triangle, 
which is due to the excitation of the quadrupole mode. The FDTD simulations show a very good agreement with the shape of the near field, but deviate from the experimentally obtained strength.

\title{
P 53: Grooves formation in steel samples with high repetition rate femtosecond pulses
}

\author{
Presenter :. Domas PAIPULAS
}

Mr. PAIPULAS, Domas (Vilnius University, Laser Research Center) Co-authors : Mr. ALESENKOV, Aleksandr (Vilnius University, Laser Research Center); Mr. KURSELIS, Kestutis (Vilnius University, Laser Research Center) ; Dr. MELNINKAITIS, Andrius (Vilnius University, Laser Research Center) ; Ms. STANKEVICIUTE, Karolina (Vilnius University, Laser Research Center) ; Dr. BALACHNINAITE, Ona (Vilnius University, Laser Research Center) ; Prof. SIRUTKAITIS, Valdas (Vilnius University, Laser Research Center)

Femtosecond laser pulses have opened up many new possibilities in laser-matter interaction and materials processing. Smaller feature sizes, greater spatial resolution, and better aspect ratios can hence be achieved. Among the various microstructures those can be manufactured utilizing laser micromachining techniques, microgroove is a key configuration that could be applied to various micro devices. We have successfully demonstrated the possibility to form 30- $200 \mu \mathrm{m}$ width grooves with depth to width ratio up to 2 and steep slopes on the steel surface by femtosecond laser ablation method. The system used in the experiments is femtosecond laser on $\mathrm{Yb}: \mathrm{KGW}$ with pulse duration $300 \mathrm{fs}$, repetition rate up to $350 \mathrm{kHz}$ and $6 \mathrm{~W}$ average power. Laser fluence, cumulative deposited energy per area, number of paths and scanning geometries were considered as variables in experiments forward for high precision and high material removal speed. The micro fabricated grooves were investigated by the means of optical microscopy, confocal profiling and SEM. In order to monitor the micromachining process laser induced breakdown spectroscopy measurements were performed. It was concluded that energy deposition rate and consequently maximal machining speed has an upper limit, which is mainly determined by the peak pulse fluence, repetition rate and material properties. Concluding from experimental results it is possible to maximize either material removal rate or energetic efficiency.

\section{P 54: Laser induced electronic transport through metallic nanostructures}

\author{
Presenter : Daniel BENNER
}

Mr. BENNER, Daniel (Universität Konstanz) Co-authors : Mr. WAITZ, Reimar (Universität Konstanz) ; Dr. GUHR, Daniel (Universität Konstanz) ; Prof. LEIDERER, Paul (Universität Konstanz) ; Prof. BONEBERG, Johannes (Universität Konstanz) ; Prof. SCHEER, Elke (Universität Konstanz)

We investigate the influence of laser light onto the electronic transport through a metallic nanoconstriction. Light-matter interaction, in particular when the matter is in the nanometer scale, is an important and steadily growing field of research due to possible applications in opto-nanoelectronics. We use lithographically fabricated Mechanically Controllable Breakjunctions (MCBJs) operated at ambient conditions for fabricating atomic-size contacts and atomically- sharp tips of gold. Detailed analysis of light-induced conductance changes has shown photo-assisted transport due to the excitation of high-energetic quasiparticles [1], collective effects such as surface plasmon excitation, and optical near fields due to antenna effects as well as thermal expansion contribute to the signal [2, 3, and 4]. We optimize the light-induced effects for the development of optoelectronic devices by studying 
systematically the effects on different metals (gold and platinum), wavelengths, position of the laser spot, and geometries (e.g. design of the nanocontacts and polarization of the laser light).

\section{P 55: Synthesis of (SrLaF)FeAs superconducting thin films by the photo excited pulsed laser deposition}

Presenter :. Satoshi KURUMI

Mr. KURUMI, Satoshi (Nihon University) Co-authors : Mr. SUGANUMA, Yoshiki (Nihon University) ; Prof. TAKANO, Yoshiki (Nihon University); Prof. SUZUKI, Kaoru (Nihon University)

The synthesis of the high Tc superconducting thin films is very important for theelectronic device use. Among Fe-based superconductors, a Co-doped SrFe2As2 epitaxialfilm is reported to become a superconductor with Tc of $20 \mathrm{~K}$. However, in order toobtain superconducting films by pulsed laser deposition (PLD), there are someproblems such as expensive substrat and high quality target materials. The photoexcited PLD (PE-PLD) is considered to have advantages for the synthesis of highquality epitaxial thin films.[1] (SrLaF)FeAs is isostructural to (LaO)FeP and(Sr0.6La0.4F)FeAs becomes superconducting below Tc of $26.3 \mathrm{~K}$. In this study, we havetried to prepare superconducting (SrLaF)FeAs thin films by PE-PLD, and investigatedthe relation between the wavelength of the photo excitation sources and thecrystallization of films. They are deposited on $\mathrm{MgO}(001)$ substrates by PLD using a Nd:YAG laser (wavelength: $532 \mathrm{~nm}$, fluence: $1.5 \mathrm{~J} / \mathrm{cm} 2$ ) with the photo excitation. Thephoto excitation sources are IR (from 0.5 to $2.5 \mu \mathrm{m}$ ), Xe (from 0.2 to $1.1 \mu \mathrm{m}$ ) andLED (peak: $400 \mathrm{~nm}$, width: $\pm 20 \mathrm{~nm}$ ). While X-ray diffraction patterns of (SrLaF)FeAs thin films excited by IR or normal PLD show only impurity phases, those excited by Xeor LED show some oriented peaks of (SrLaF)FeAs. This result indicates that theexcitation by ultra violet rays increases a reaction ability and has an effective role of the growth of the superconducting epitaxial films.

[1] T. Takayama et al.: Appl. Phys. A in print

\section{P 56: Ionic liquids: Novel matrices for improving the photostabilities of laser dyes}

Presenter : Jennifer KRAUSE

Ms. KRAUSE, Jennifer (1.Physikalisches Institut / Universität zu Köln) Co-authors : Dr. WOIKE, Theo (I. Physikalisches Institut, Universität zu Köln) ; Prof. SCHANIEL, Dominik (Laboratoire de Cristallographie, Institut Jean Barriol, Faculté des Sciences et Technologies, Nancy Université) ; Dr. PITULA, Slawomir (Anorganische Chemie I (Festkörper und Materialien), Ruhr-Universität Bochum) ; Prof. MUDRING, Anja-verena (Anorganische Chemie I (Festkörper und Materialien), Ruhr-Universität Bochum) ; Prof. IMLAU, Mirko (Fachbereich Physik, Universität Osnabrück)

Organic dyes are widely used in labeling and staining purposes. Their low price, high quantum efficiency and their exceptional wide emissive range also predestine these compounds for applications e. g. LASER spectroscopy and light emitting technical devices. In order to produce stable laser-dye solutions for DYE-LASERs or other optical applications we dissolved commercially available dyes in task-specific hydrophobic and thermally stable ionic liquids. Ionic liquids consist of organic cations and organic or inorganic anions. This liquid salts are thermally stable, notflammable and have a very low, barely measurable vapor pressure. We embedded quenchers into the ionic-liquid-dye solution and removed the dissolved oxygen. This resulted in a complete suppression of laser-dye degradation under 
laser irradiation. The such achieved stability of the ionic-liquid-dye solutions enables us to build dye lasers without the need for a pumping system and reservoirs. We measure the luminescence and absorption properties as well as the photostability behaviour of different ionic-liquid-dye solutions. In order to investigate the underlying photophysical processes that lead to the photodegradation of the dye -molecules, electron spin resonance and cyclic voltammetry measurements.

\section{P 57: Synthesis and characterization of ZnO thin films by 532 nm@40 ps laser pulses}

Presenter :. Carmen RISTOSCU

Dr. RISTOSCU, Carmen (National Institute for Lasers, Plasma and Radiation Physics) Co-authors : Prof. MIHAILESCU, Ion N. (National Institute for Lasers, Plasma and Radiation Physics) ; Dr. JAFER, Rashida (Università degli Studi di Milano- Bicocca) ; Dr. AL-HADEETHI, Yas (Università degli Studi di Milano- Bicocca) ; Prof. BATANI, Dimitri (Università degli Studi di Milano-Bicocca)

The synthesis by pulsed laser deposition technique of $\mathrm{ZnO}$ thin films with appropriate characteristics for gas sensing applications is herein reported. We used a Quanta Nd:YAG laser system capable of delivering a linearly P-polarised $1064 \mathrm{~nm}$ laser radiation with an energy per pulse of $120 \mathrm{~mJ}$ in $40 \mathrm{ps}$ (FWHM). To generate the plasma, $532 \mathrm{~nm}$ laser beam irradiates the $\mathrm{ZnO}$ target placed inside the vacuum chamber evacuated down to 10-3 torr. The incident laser fluence was of $28 \mathrm{~J} / \mathrm{cm} 2$ in a spot of $10 \mathrm{~mm} 2$. The ablated material was collected onto double polished (111) Si or quartz wafers placed at a separation distance of $7 \mathrm{~mm}$. The AFM, UV-Vis and FT-IR results indicated that we obtained pure $\mathrm{ZnO}$, with a uniform surface having an average roughness of $37 \mathrm{~nm}$. The average transmission in the Vis-IR spectral region was found to be higher than $85 \%$. The good optical properties stand for the basic requirements which allow the implementation of the obtained thin films as planar waveguides in optical gas sensors. The results of these investigations will be compared to the ones obtained when using ns laser pulses and their potential as optical sensors will be critically discussed.

\section{P 58: Laser-induced breakdown spectroscopy for the analysis of boron in iron-oxides prepared under hot steam condition}

Presenter : Dr. E.C. JUNG

Dr. YEON, J.w. (Korea Atomic Energy Research Institute) Co-authors : Dr. JUNG, E.c. (Korea Atomic Energy Research Institute) ; Mr. JUNG, S-h. (Korea Atomic Energy Research Institute) ; Mr. WHANG, J. (Korea Atomic Energy Research Institute) ; Dr. SONG, K (Korea Atomic Energy Research Institute)

The leakage of reactor coolant at the pressure boundaries is one of the emerging issues in pressurized water reactors. Since the major chemical additive in the coolant is a boric acid, it is of interest to analyze the presence of the boron (B) in the deposits found at the pressure boundaries for the examination of coolant leakage. Due to the high radioactivity of the deposits, however, the conventional method takes a long time and is costly to detect the coolant leakage. Laser-induced breakdown spectroscopy (LIBS) can provide several advantages in terms of a localized microanalysis of oxides, and minimizing a sample preparation prior to a quantitative analysis. In addition, the remote capabilities of LIBS in conjunction with a fiber optic cable make it possible to perform an in-situ analysis of radioactive materials in hazardous environment. In this work, LIBS was applied to the 
detection of $\mathrm{B}$ embedded in iron (Fe) oxides in order to develop a coolant leakage detection system. Laser-induced plasma was generated by focusing a third harmonic of Nd:YAG laser onto oxide samples. Several iron-oxides containing different boron concentrations were prepared under hot steam condition. The ratio of B to Fe was measured by comparing the intensity of the B emission at 249.773 $\mathrm{nm}$ with that of the $\mathrm{Fe}$ emission at $250.113 \mathrm{~nm}$ as an internal standardization. It was confirmed that the limit of detection of B concentration in the iron-oxides was about 0.01 wt. \%

\section{P 59: In-plane detection of fluorescence signals in microfludic lab-on-chip flow cytometry}

Presenter :. Casper KUNSTMANN-OLSEN

Mr. KUNSTMANN-OLSEN, Casper (Mads Clausen Institute, University of Southern Denmark) Co-authors : Dr. HOYLAND, James (Mads Clausen Institute, University of Southern Denmark) ; Prof. RUBAHN, Horst-günter (Mads Clausen Institute, University of Southern Denmark)

The most common configuration used in traditional flow cytometers is a threedimensional one with scattering and fluorescence detection orthogonal to each other and to the fluid stream. However, keeping to an entirely planar device geometry has great advantages in terms of overall simplicity and cost of construction. Here, several means of extracting fluorescence signals from flowing cell suspensions in a single plane are examined. Simple microfluidic flow-cytometer structures incorporating lateral hydrodynamic focusing were molded in PDMS. Several geometries for embedding optical fibers and custom molded waveguides into the same structure were compared. These light guides are used both for the excitation laser and for detecting scatter and fluorescence from the particles.The low numerical aperture of a bare multi-mode fiber leads to low efficiency of light gathering. Improvement in light yield is demonstrated with molded cylindrical lenses and by using

channels filled with high refractive index polymers as waveguides.

\section{P 60: Ultra-short pulse laser ablation of copper, silver and tungsten: experimental data and two- temperature model simulations}

Presenter : Juha-matti SAVOLAINEN

Mr. BYSKOV-NIELSEN, Jeppe (Department of Physics and Astronomy, Faculty of Science, Aarhus University) Co-authors : Mr. SAVOLAINEN, Juha-matti (Department of Physics and Astronomy, Faculty of Science, Aarhus University) ; Mr. SNOGDAHL CHRISTENSEN, Martin (Department of Physics and Astronomy, Faculty of Science, Aarhus University) ; Dr. BALLING, Peter (Department of Physics and Astronomy, Faculty of Science, Aarhus University)

Experimental results of femtosecond laser ablation of the metals copper, silver and tungsten are compared with simulations based on the two-temperature model. The comparison provides new information about the laser-heating process: For the noble metals $(\mathrm{Cu}, \mathrm{Ag})$ energy transport via ballistic electrons must be included, while this effect is negligible for a transition metal (W). The model calculation is also employed to investigate the dependence of the threshold fluence and melting depth on pulse duration. It is observed that for pulses shorter than approximately 1 ps the threshold fluence and melting depth are independent on the pulse duration, while they increase as $\operatorname{tau}^{\wedge} 0.47 \mathrm{and}^{\mathrm{tau}} \mathrm{ta}^{\wedge} 0.51$, respectively, for pulses longer than $\sim 40 \mathrm{ps,} \mathrm{in} \mathrm{good} \mathrm{agreement} \mathrm{with} \mathrm{approximate} \mathrm{analytical} \mathrm{expressions}$ predicting a sqrt(tau) dependence. 


\section{P 61: Stability of free flying fluid films}

Presenter : Pascal FRANK

Mr. FRANK, Pascal (University Konstanz) Co-authors : Prof. BONEBERG, Johannes (University Konstanz) ; Prof. LEIDERER, Paul (University Konstanz)

A liquid sheet without contact to any substrate is hardly stable. However, the generation of free fluid films, that are stable for some hundred nanoseconds, is feasible through laser ablation. To prepare these films, a $10 \mathrm{~ns}$ laser pulse is used to rapidly heat the surface of a silicon substrate, where in advance a thin $(100 \mathrm{~nm}-1 \mu \mathrm{m})$ layer of liquid is condensed. The subsequent evaporation of a thin layer of the liquid at the interface leads to the desorption and acceleration of a freestanding film, whose position is tracked by ns-time resolved reflectometry. The measurements show that under ambient conditions the flight is nearly parabolic and the film returns to the substrate after some hundred nanoseconds as the accelerating pressure decreases below the ambient pressure; the maximum distance of the film is a few micrometers. If the exciting laser pulse is modulated via laser interference with periods exceeding the thermal diffusion length during the pulse, the vapour which accelerates the film does not form uniformly. Up to a certain extent of laser energy this does not lead to complete rupture of the film, but to a modulation during the time of flight. Investigation of the diffraction provides insight into the stability of this modulation.

\section{P 62: Synthesize of photocatalytic SrxLa1-xTiO3 film for hydrogen generation with visible area in solar light excitation by pulsed laser deposition}

Presenter : Takayuki KURIHARA

Mr. KURIHARA, Takayuki (Nihon University) Co-authors : Mr. KURUMI, Satoshi (Nihon University) ; Mr. SEKI, Keisuke (Nihon University) ; Ms. NAKADA, Tomoko (Nihon University) ; Dr. KAMIMOTO, Atsushi (Nihon University); Dr. HIROSE, Hideharu (Nihon University) ; Dr. MASUTANI, Shigeyuki (Nihon University) ; Prof. SUZUKI, Kaoru (Nihon University)

La doped $\mathrm{TiO} 2$ have attracted great interest for photocatalytic properties, which can be used visible area in solar light although only $\mathrm{TiO} 2$ limiting with ultra violet area. However, these reports were almost powdered La2Ti2O7. To circumvent this problem, we have attempted to synthesized La doped $\mathrm{TiO} 2$ thin film on quartz substrate by pulsed laser deposition using non-sintered target. In addition we have tried to Sr doped as imparity for improvement only hydrogen generation. These films were composed of several molar ratios. We have successfully crystallized perovskite structure films which were $\mathrm{La}$ doped $\mathrm{TiO} 2$ thin film of La2Ti2O7, $\mathrm{Sr}$ doped $\mathrm{TiO} 2$ thin film of $\mathrm{SrTiO} 3$ and both impurity doped thin film of SrxLa1-xTiO3 $(\mathrm{x}=0.1 \sim 0.9)$. The best of hydrogen generation efficiency was $16 \mu \ell / \mathrm{hcm} 2$ by $\mathrm{Sr} 0.7 \mathrm{La} 0.3 \mathrm{TiO} 3$ to $\mathrm{SrTiO3}$, and furthermore, the band gap of Sr0.3La0.3TiO3 was $3.2 \mathrm{eV}$ which showed the same tendency. As a result, the molar ratio of $\mathrm{Sr} 0.7 \mathrm{La} 0.3 \mathrm{TiO} 3$ thin film confirmed suitable to hydrogen generation. 


\section{P 63: Single pulse laser interference lithography}

Presenter : Stephen RIEDEL

Mr. RIEDEL, Stephen (Universität Konstanz, Germany) Co-authors : Dr. GELDHAUSER, Tobias (Research Institute for Electronic Science, University of Hokkaido, Sapporo, Japan) ; Mr. SCHMOTZ, Markus (Universität Konstanz, Germany) ; Prof. LEIDERER, Paul (Universität Konstanz, Germany) ; Prof. BONEBERG, Johannes (Universität Konstanz, Germany)

We present a technique which can be used to create periodic topographic structures as well as periodic surface energy patterns. As a direct structuring method no resists or subsequent etching steps are needed in the patterning process. In our experiments we use a nanosecond pulsed Nd:YAG laser, divide the beam into multiple parts and redirect them on the sample surface in order to get a periodic intensity distribution. For metal and semiconductor thin films we can achieve with this technique, arrays of hills or holes with periodicities between $140 \mathrm{~nm}$ to $30 \mu \mathrm{m}$. Based on our measurements we propose a model of the not yet fully understood structuring process. Finally in taking advantage of the threshold behavior of the thermal desorption of a Self Assembled Monolayer (SAM) we can create periodic surface energy pattern for subsequent segregation of polymer blends or selective binding of specific molecules.

\section{P 64: Imaging the Near Field of Dielectric and Metallic Nanoparticles}

Presenter :. Andreas KOLLOCH

Mr. KOLLOCH, Andreas (University of Konstanz, Germany) Co-authors : Dr. GELDHAUSER, Tobias (Research Institute for Electronic Science, University of Hokkaido, Sapporo, Japan) ; Mr. KüHLER, Paul (University of Konstanz, Germany) ; Dr. SIEGEL, Jan(Instituto de Óptica, CSIC, Madrid, Spain) ; Dr. SOLíS, Javier (Instituto de Óptica, CSIC, Madrid,Spain) ; Prof. AFONSO, Carmen N. (Instituto de Óptica, CSIC, Madrid, Spain) ; Prof. GARCíA DEABAJO, F. Javier (Instituto de Óptica, CSIC, Madrid, Spain) ; Prof. UENO, Kosei (Research Institute for Electronic Science, Hokkaido University, Sapporo, Japan / PRESTO, Japan Science and Technology Agency, Kawaguchi, Japan) ; Prof. MISAWA, Hiroaki (Research Institute for Electronic Science, Hokkaido University, Sapporo, Japan) ; Prof. BONEBERG, Johannes (University of Konstanz) ; Dr. PLECH, Anton (Institut für Synchrotronstrahlung, Karlsruher Institut für Technologie, Karlsruhe, Germany) ; Prof. LEIDERER, Paul (University of Konstanz, Germany)

Focussing light down to the nanometer scale is important for a wide range of applications ranging from the local modification of a substrate to highly effective optical pumping of nanoscopic structures (e.g. quantum dots, molecules). The interaction of light with mesoscopic to nanoscopic objects gives rise to different kinds of focussing effects. In the case of dielectric spherical particles larger than or comparable to the wavelength of the illuminating laser pulse, the particle simply acts as a lens, focusing the light onto the surface underneath. The minimal focal spot which can be achieved in this way has a diameter of about $\lambda / 4$. A larger compression of the light field well below 100 $\mathrm{nm}$ can be obtained by using metallic nanoparticles. When illuminated by light, these nanoparticles act as antennas which provide near field enhancements confined to a very small space (down to a few nanometres). For the application of the near field as a tool in science and technology, a profound understanding of the field distribution of this range of structures is crucial. We present several examples for the near field distribution of micro- and nanoscopic objects obtained by two different imaging techniques and compare them to calculations. For dielectric particles, the near field has been measured using the phase change in GST (Ge2Sb2Te5) while the near field of the smaller metallic 
structures was depicted by local ablation on a silicon substrate.

\section{P 65: Structure of nano-hole array formed on GaN substrate using femtosecond laser ablation in viscous liquid}

Presenter : Takuma ITO

Mr. ITO, Takuma (RIKEN, Tokyo Denki University) Co-authors : Dr. NAKASHIMA, Seisuke (RIKEN) ; Prof. UGIOKA, Koji (RIKEN) ; Prof. TAKAI, Hiroshi (Tokyo Denki University) ; Prof. MIDORIKAWA, Katsumi (RIKEN)

Gallium nitride $(\mathrm{GaN})$ is known as a wide bandgap semiconductor which is used for blue/UV LEDs. Since GaN possesses quite high refractive index ( 2.5) compared with air, a light extraction efficiency of GaN based LED is limited to only a few percent. Surface modifications such as forming 2-D photonic crystals are expected for improvement of the light extraction efficiency. At present, reactive ion etching (RIE) process is mainly used for precise fabrication, in spite of the low etching rate of RIE. In the mean while, we have investigated GaN processing using a femtosecond(fs) laser ablation technique, which enables high speed etching. We have reported that the ablation reaction in $\mathrm{HCl}$ acid solution result in higher ablation quality compared with that in air even after post-etching process. Moreover, we have also achieved to fabricate ablation craters with high aspect ratio by irradiation of multiple laser pulses. Further enhancement of fabrication resolution is, however, a still remaining issue. In this paper, we investigate the fs laser ablation in viscous liquid with different viscosity to improve the resolution. An fs laser (wavelength: $387 \mathrm{~nm}$ (SHG), pulse width: $150 \mathrm{fs}$ ) is focused onto oil immersed GaN substrate with an objective lens(NA:1.4). At pulse energy of 3nJ, we obtained nanometer-size holes with the diameter of around 100nm(at FWHM). This result clearly demonstrates that the fabrication resolution was significantly improved compared with that of our previous work.

\section{P 66: Investigation of optical properties in near-zero-permittivity operation range for a superconducting photonic crystal}

Presenter : Prof. Tzong-jer YANG

Prof. YANG, Tzong-jer (Department of Electrical Engineering, Chung Hua University) Co-authors : Mrs. CHEN, Meisoong (Chung Hua University) ; Prof. WU, Chien-jang (National Taiwan Normal University)

Optical properties of a periodic multilayer structure containing a superconducting material are theoretically investigated based on the Abeles theory for a stratified medium and the twofluid model for a superconductor. It is found that there are somereflection dips within the highreflection band near the superconducting threshold wavelength at oblique incidence for TM wave, but they are not seen for TE wave. These dips behave like the strongly localized passbands, which provide a feasible way of designing a narrowband resonator without physically introducing any defect layer in the structure. 


\section{P 67: Ultrafast laser ablation and micromachining of selected group IV materials}

Presenter : E. M. HSU

HSU, E. M. (Department of Engineering Physics, McMaster University, Hamilton, Canada) Co-authors :MAILMAN, N. A. (Department of Physics and Astronomy, McMaster University, Hamilton, Canada) ; BAYER, B. C. (Department of Engineering, University of Cambridge, Cambridge, UK) ; BRAWLEY-HAYES, J. A. Z. (Department of Physics and Astronomy, McMaster University, Hamilton, Canada) ; AN, R. (Department of Engineering Physics, McMaster University, Hamilton, Canada) ; HOFMANN, S. (Department of Engineering, University of Cambridge, Cambridge, UK) ; ROBERTSON, J. (Department of Engineering, University of Cambridge, Cambridge, UK) ; BOTTON, G. A. (Department of Materials Science and Engineering, McMaster University, Hamilton, Canada) ; KNIGHTS, A. K. (Department of Engineering Physics, McMaster University, Hamilton, Canada) ; JESSOP, P. E. (Department of Engineering Physics, McMaster University, Hamilton, Canada) ; HAUGEN, H. K. (Department of Engineering Physics and Department of Physics and Astronomy, McMaster University, Hamilton, Canada)

We present studies of ultrafast laser ablation and micromachining of selected group IV materials, including multiple-wall carbon nanotube (MWNT) forests, single-crystal synthetic diamond, as well as crystalline silicon. Preliminary results of ultrafast laser micromachining of MWNT forests show features that are indicative of the modification and removal mechanisms upon laser irradiation, as well as the interplay of laser and MWNT forest parameters. Ultrafast laser irradiation of single-crystal synthetic diamond generated sub-wavelength periodic surface structures at the incident surface as well as the rough side surface where the laser focus translated out of in bulk experiments. The ejected material was also studied, and the results indicate laser ablation as a potential mean to generate diamond nanoparticles. Surface irradiation of crystalline silicon generated periodic ripples and quasiperiodic conical structures. We are exploring the feasibility of using these features in improving silicon based photonics devices. We also present findings on the threshold and incubation behavior of ultrafast laser ablation of silicon samples with and without pre-irradiation with an ion beam. Initial characterization showed that ablation thresholds of silicon can be altered by controlling the ion implantation parameters.

\section{P 68: Investigating a thin gold foil under femtosecond laser irradiation.}

Presenter :. Fernande FOTSA NGAFFO

Dr. FOTSA NGAFFO, Fernande (University of Buea) Co-authors : Mr. FONKI MEOTO, Emile (University of Buea) ; Dr. DIKANDE, Alain Moise (University of Buea)

The interaction of an ultrashort UV laser pulse with a metal target has been vigorously studied over the last 37 years preceding the Parabolic Two-Step (PTS) model suggested by Anisimov et al. [1]. Describing the laser source term mathematically is not a straightforward task. Many authors have used source terms of the form $S(z, t)=(1-R) I(t) f(z)$, where the intensity $I(t)$ is a Gaussian and the attenuation $\mathrm{f}(\mathrm{z})$ an exponential decay. In this report the same term has been used, but with an extra factor added so as to account for the finite nature of the metal target. We then employ the analytical method suggested by Al Nimr et al.[2]. A simple perturbation is used to uncouple the parabolic system of equations, and then a Green's function is used to solve the uncoupled equations for the electronic temperature. We then simulate the effect of the extra factor on the electronic peak temperature and the thermalisation time. We show that it doesn't significantly affect the nonequilibrium between the 
electronic and lattice subsystems.

\section{P 69: Band-pass filters for $\mathrm{THz}$ spectral range fabricated by laser ablation}

Presenter :. Gediminas RACIUKAITIS

VOISIAT, Bogdan (Center for Physical Science and Technology) Co-authors : Dr. RACIUKAITIS, Gediminas (Center for Physical Science and Technology) ; Dr. KAšALYNAS, Irmantas (Center for Physical Science and Technology)

We report on the resonant $\mathrm{THz}$ band filters fabricated employing the ultrashort-pulse UV laser milling process, when focused laser beam was used to ablate the material. The picosecond laser PL10100TH (Ekspla Ltd.), radiating pulses of 10 ps duration with the $100 \mathrm{kHz}$ pulse repetition rate and the $355 \mathrm{~nm}$ wavelength was used as an energy source. The laser direct write system was employed to cut matrixes of cross-shape holes in the $30-\mu \mathrm{m}$-thick stainless steel foil and the molybdenum thin film $(0.5 \mu \mathrm{m})$ layer deposited on polyimide film with the thickness of $25 \mu \mathrm{m}$. Different laser processing strategies were applied: holes were cut through in the metal foil and molybdenum film was removed from the polymer substrate by ablation. The band-pass filters for the center frequency at $0.693,1.40$, and $1.63 \mathrm{THz}$ (respectively named as 1, 2, and 3) were designed. The filter performance was determined by the mesh period, cross-arm length, and width. The regular shape, smoothness of edges and sharpness of corners were the main attributes for the quality assessment of the laser-fabricated cross-shape holes. The terahertz time-domain spectroscopy and conventional spacedomain Fourier Transform spectroscopy were used to specify filters performance. Experimental data were supported by three-dimensional finite-difference time-domain (FDTD) simulations.

\section{P 70: Templates for integrated nanofiber growth}

Presenter :. Roana Melina De Oliveira HANSEN

Mrs. HANSEN, Roana Melina De Oliveira (Mads Clausen Institute, University of Southern Denmark) Co-authors Dr. KJELSTRUP-HANSEN, Jakob (Mads Clausen Institute, University of Southern Denmark) Prof. RUBAHN, Horstgünter (Mads Clausen Institute, University of Southern Denmark)

Organic nanofibers made from para-hexaphenylene (p6P) molecules [1] exhibit a range of useful optical properties with potential applications in future submicron-sized opto-electronics and photonics. For example, the nanofibers display intense, anisotropic luminescence, waveguiding, and lasing. Typically, the nanofibers are made by physical vapor deposition of $\mathrm{p} 6 \mathrm{P}$ molecules onto a heated mica substrate in a high vacuum environment, which leads to mutually parallel nanofibers with tailored dimensions [1]. However, technological use of the nanofibers calls for the ability to grow on other substrate materials and to control the position and growth direction for in-situ growth. Besides such single-crystalline templates, the nanofibers can also be grown on non-crystalline gold surfaces [2], on which the orientation of the nanofibers can be manipulated by structuring the gold surface prior to p6P deposition [2]. In this work it is demonstrated, how such organic nanofibers can be grown across small gaps between gold electrodes, whereby electrical contact to in-situ grown p6P nanofibers is achieved. Furthermore, the electrical characteristics of in-situ grown p6P nanofibers are analyzed. 


\section{P 71: Angular distribution anisotropy of fragments ejected from methyl iodide clusters}

Presenter : Gabriel KARRAS

Mr. KARRAS, Gabriel (Department of Physics, University of Ioannina,)Co-authors : Dr. KOSMIDIS, C. (Department of Physics, University of Ioannina,)

Molecular clusters are a lay-over in the way between isolated molecules to condensed matter. Their interaction with strong electromagnetic fields created by lasers led to the observation of highly charged, energetic ions and electrons, emission of x-rays and neutrons. Furthermore high order harmonic generation has been observed from laser-cluster interaction and, as reported recently, with larger efficiency compared to bulk matter.3 Methyl iodide clusters have been paid special attention due to the large amount of data existing in the literature concerning the photochemistry of the corresponding monomers. In the current work we present experimental results acquired from the irradiation of methyl iodide clusters with femtosecond laser pulses. Neutral clusters of CH3I are formed in a supersonic molecular beam and their ionization is induced by a 20fs Ti: Sapphire laser. The ionic signal is investigated under different experimental conditions, such as polarization (linear and circular) and laser intensity by means of a linear time-of- flight mass spectrometer. The angular distribution of the fragment ions was recorded and three different angular distributions were identified, one isotropic and two anisotropic. Their dependence on the laser intensity has been studied. There is strong evidence that the ions exhibiting anisotropic angular distribution with a maximum in the direction parallel to the laser polarization vector are produced via an electron impact ionization process.

\section{P 72: Periodic surface structure behaviour on polyimide investigated by temporally tailored laser pulses}

Presenter : Magdalena FORSTER

Ms. FORSTER, Magdalena (University of Vienna, Department of Physical Chemistry, Waehringer Strasse 42, A-1090 Wien, Austria), Mr. FAURE, Nicolas (Université Jean Monnet, Laboratoire Hubert Curien, F-42000 Saint Etienne, France),Prof. AUDOUARD, Eric (Université Jean Monnet, Laboratoire Hubert Curien, F-42000 Saint Etienne, France), Prof. STOIAN, Razvan (Université Jean Monnet, Laboratoire Hubert Curien, F-42000 Saint Etienne, France), Prof. KAUTEK, Wolfgang (University of Vienna, Department of Physical Chemistry, Währinger Str. 42, A-1090 Vienna, Austria)

Femtosecond laser treatment of solids leads to so-called ripples with sub-micrometer periodicities. Applications of ripples range from micromachining to the generation of self-cleaning surfaces similar to that of the Lotus leaf. The mechanism of the formation is still subject to discussions. Periodic surface structures with periodicities close to the laser wavelength were observed on polyimide upon femtosecond laser treatment. Their behaviour was investigated by temporally tailored pulses. They occur at more than five $150 \mathrm{fs}$ pulses parallel to the electric field. Beyond pulse durations of $500 \mathrm{fs}$, these ripples turned into chaotic surface features. When using sequences of double pulses an increase of the double pulse delay time above 2 ps caused a transition from ordered to disordered structures as well. The observed behaviour is discussed in terms of electronic soliton excitations. 


\section{P 73: Cold atoms near surfaces}

Presenter: Jacek Fiutowski

Fiutowski, Jacek, Bartoszek, D., Kawalec, T, T. Dohnalik, T., Rubahn, H.-G., Mads Clausen Institute, NanoSYD, University of Southern Denmark, Alsion 2, 6400 Sonderborg, Denmark and Marian Smoluchowski Institute of Physics, Jagiellonian University, Reymonta 4, 30-059 Cracow, Poland

One method of assembling a large number of neutral atoms near a solid surface is to use surface dipole traps - magnetic and optical. The basic element of the latter are so-called 'optical mirrors'. The atoms to be trapped for most of them are delivered by magnetooptical traps. An optical mirror can be constructed from a dielectric prism (usually glass or quartz), on the surface of which an evanescent wave is excited with a frequency greater than the frequency of selected optical transitions in reflected atoms. In our recently developed optical dipole mirror for rubidium atoms we investigate main aspects of radiation pressure on bouncing atoms exerted by the evanescent wave - both far-, blue-detuned and close to resonance. In addition we present briefly some further applications, like measurements with prisms coated with thin gold films, "optical atom chips" for trapping and/or acting on cold atoms near surfaces, alternative plasmon sources etc.. 